Electronic Supplementary information for:

\title{
Effect of Distortions on the Geometric and Electronic Structures of One-electron Oxidized Vanadium(IV), Copper(II) and Cobalt(II)/(III) Salen Complexes
}

Hussein Kanso, ${ }^{a}$ Ryan M. Clarke, ${ }^{\mathrm{b}}$ Amélie Kochem, ${ }^{\mathrm{a}}$ Himanshu Arora, ${ }^{\mathrm{a}}$ Christian Philouze, ${ }^{\mathrm{a}}$ Olivier Jarjayes, ${ }^{a}$ Tim Storr $^{b}$ and Fabrice Thomas ${ }^{a}$

a. Univ. Grenoble Alpes, CNRS, DCM, F-38000 Grenoble, France.

b. Department of Chemistry, Simon Fraser University, Burnaby, British Columbia, V5A 1S6, Canada.

Table of content:

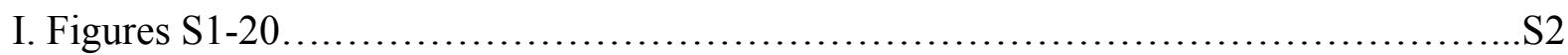

II. Tables...................................................................... 15

III. Computational details...................................................S16 


\section{Figures}

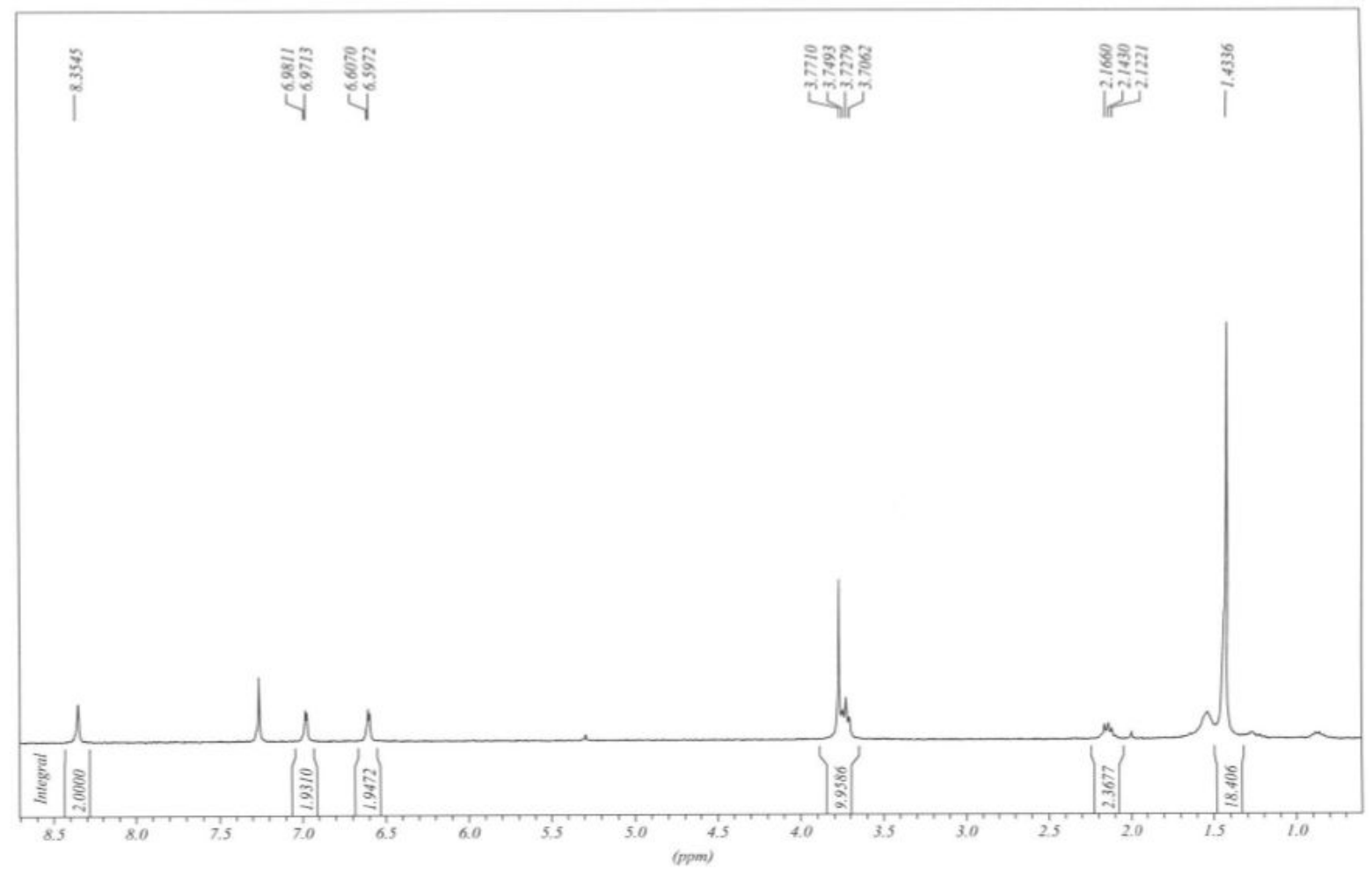

Fig. S1 ${ }^{1} \mathrm{H}$ NMR spectrum of the $N, N^{\prime}$-bis(3-tert-butyl-5-methoxysalicylidene)-1,3-propanediamine (300 MHz, $\mathrm{CDCl}_{3}, 298 \mathrm{~K}$ )

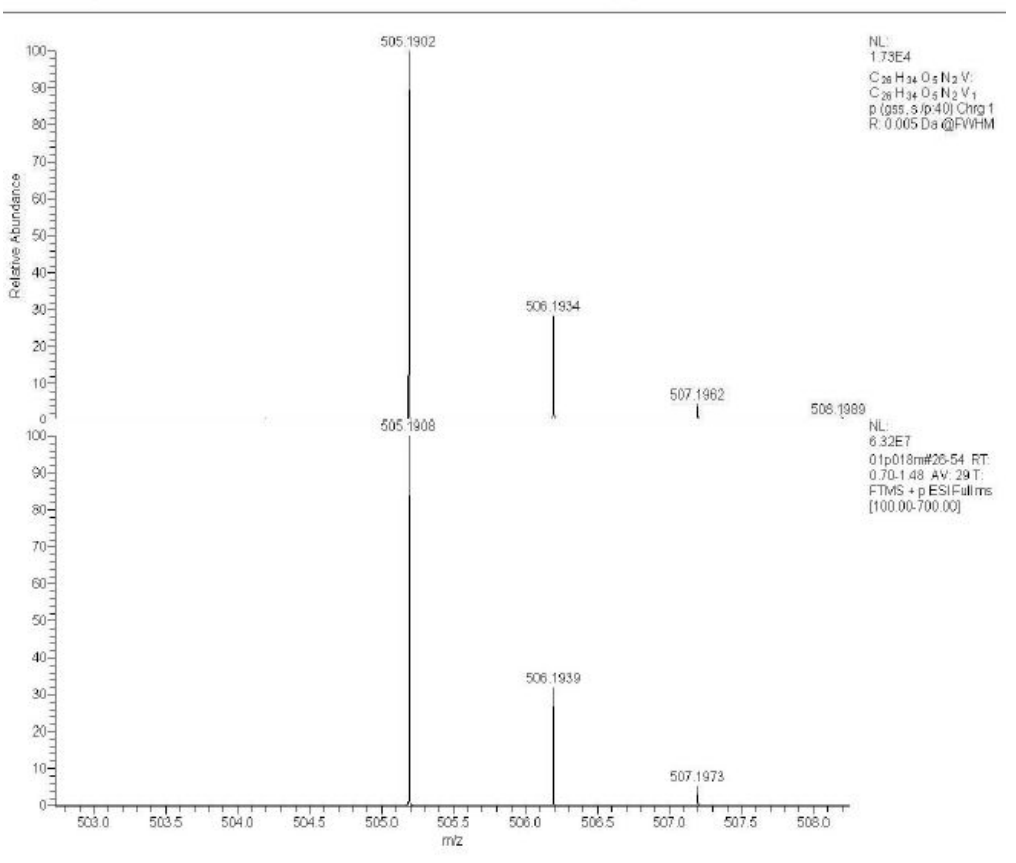

Fig. S2 HRMS spectrum of 1 (top: calculated, bottom: experimental) 


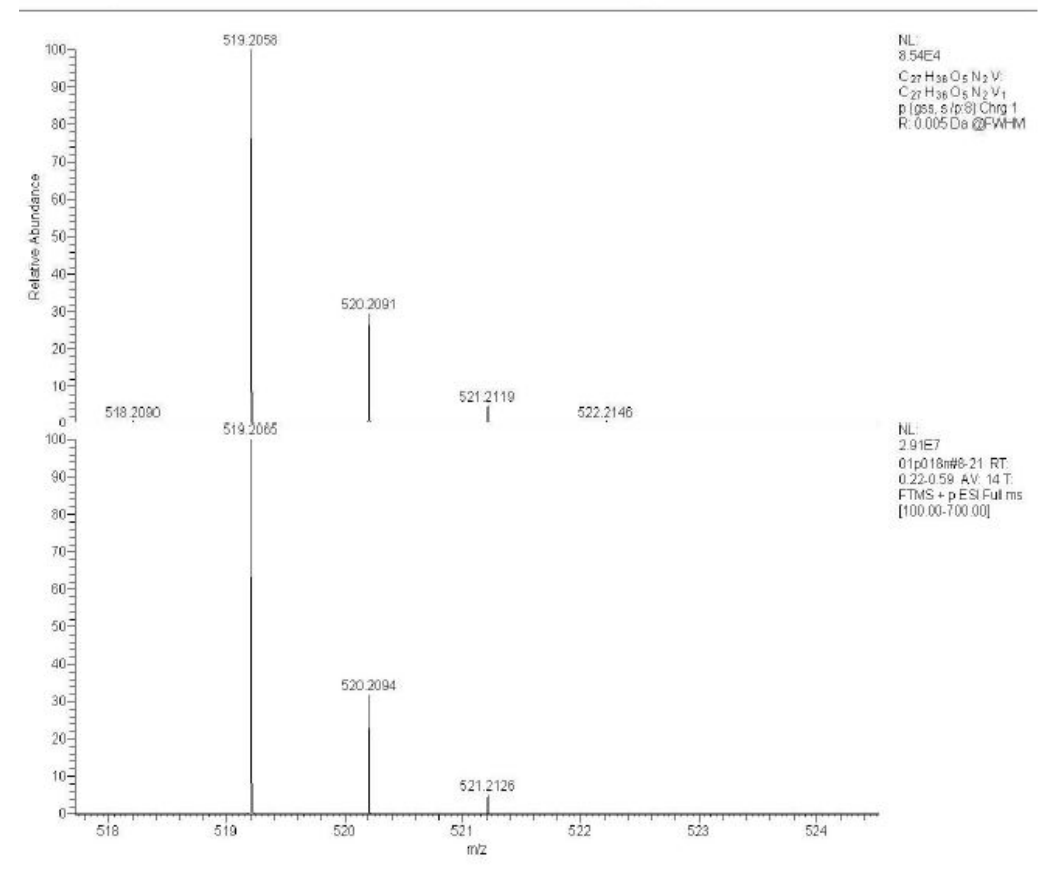

Fig. S3 HRMS spectrum of $\mathbf{2}$ (top: calculated, bottom: experimental)

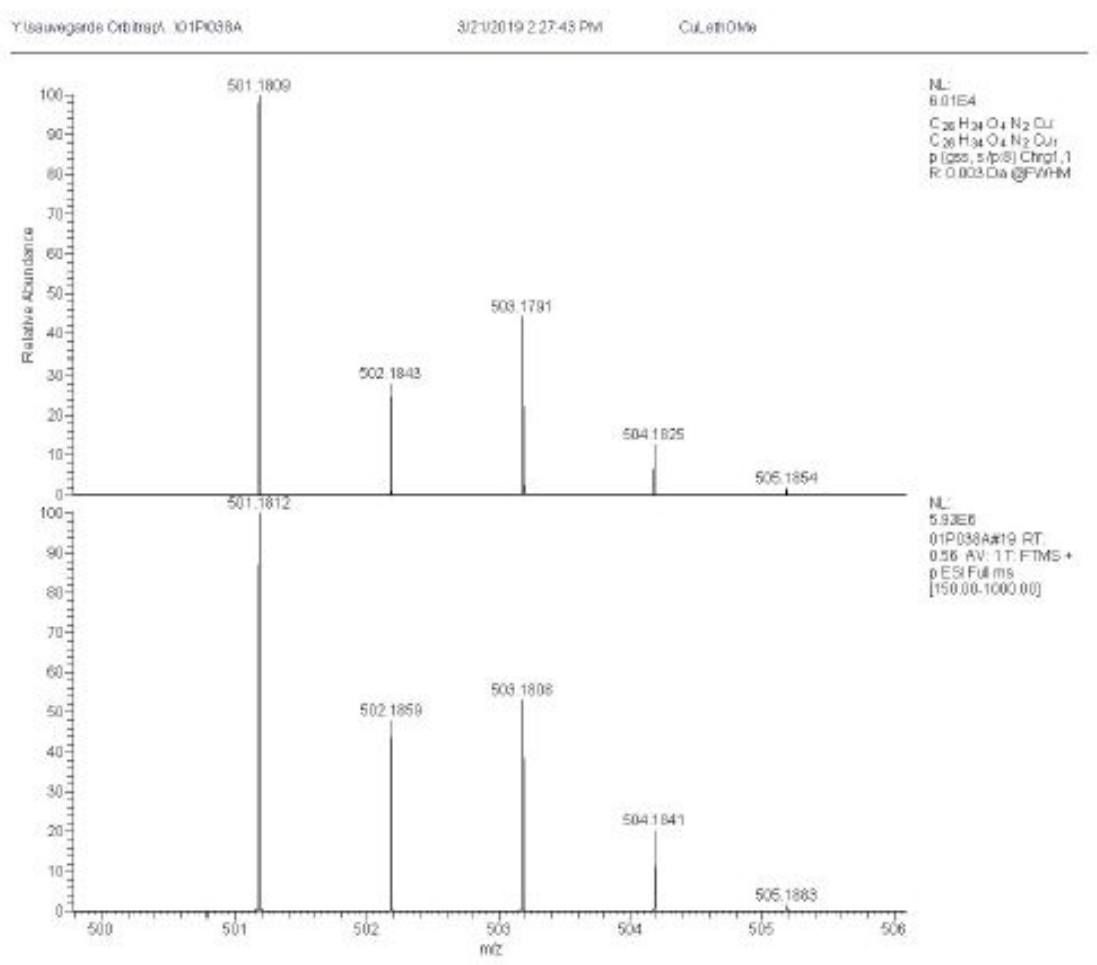

Fig. S4 HRMS spectrum of $\mathbf{3}$ (top: calculated, bottom: experimental) 


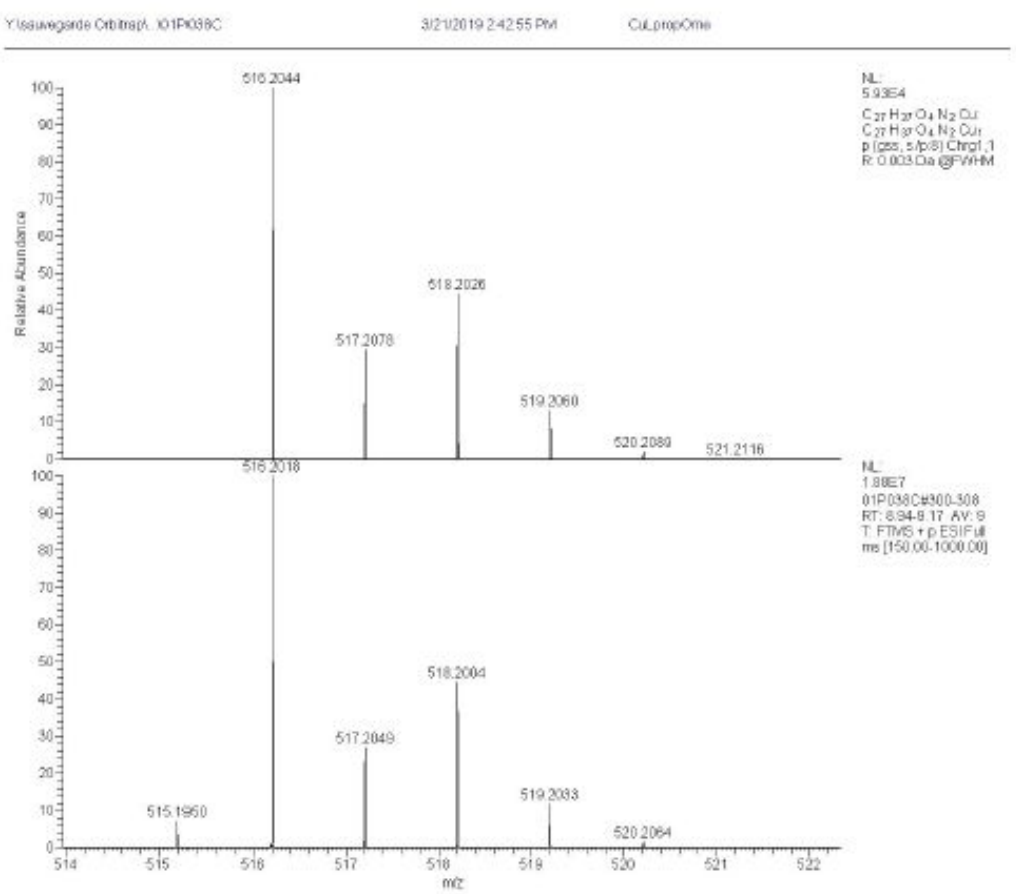

Fig. S5 HRMS spectrum of $\mathbf{4}$ (top: calculated, bottom: experimental)

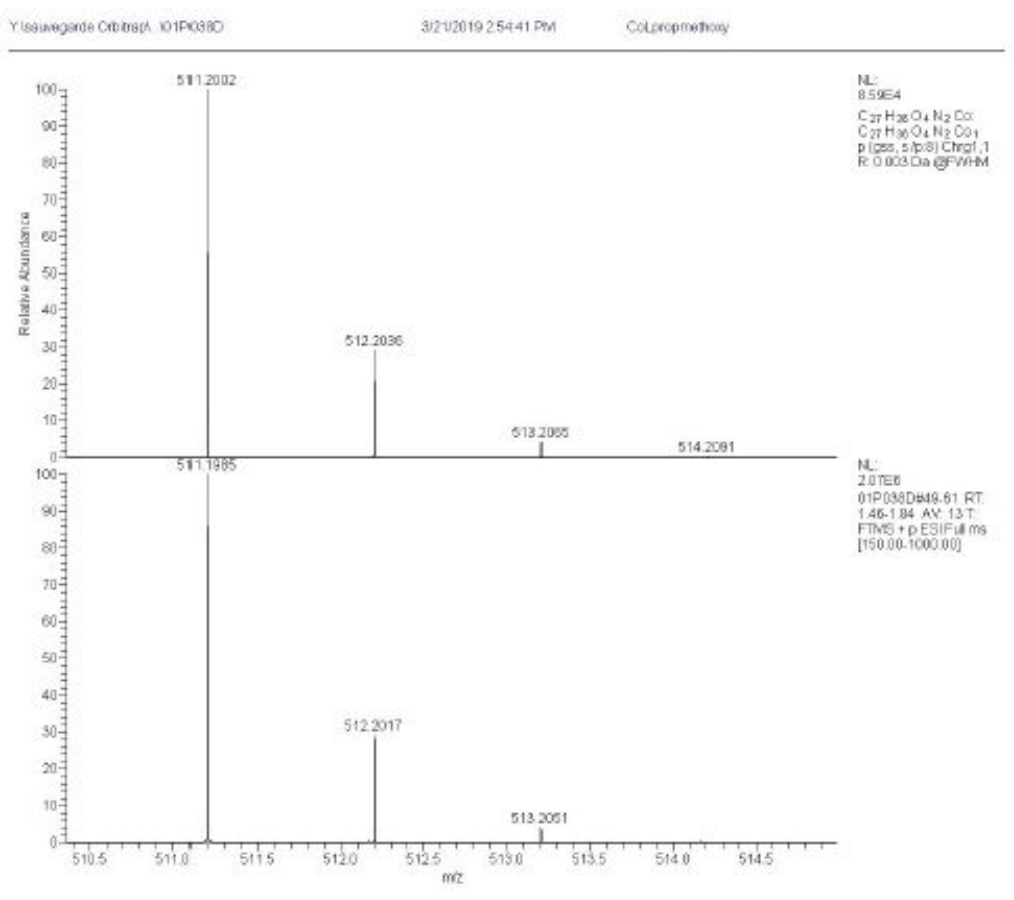

Fig. $\mathbf{S 6}$ HRMS spectrum of 6 (top: calculated, bottom: experimental) 


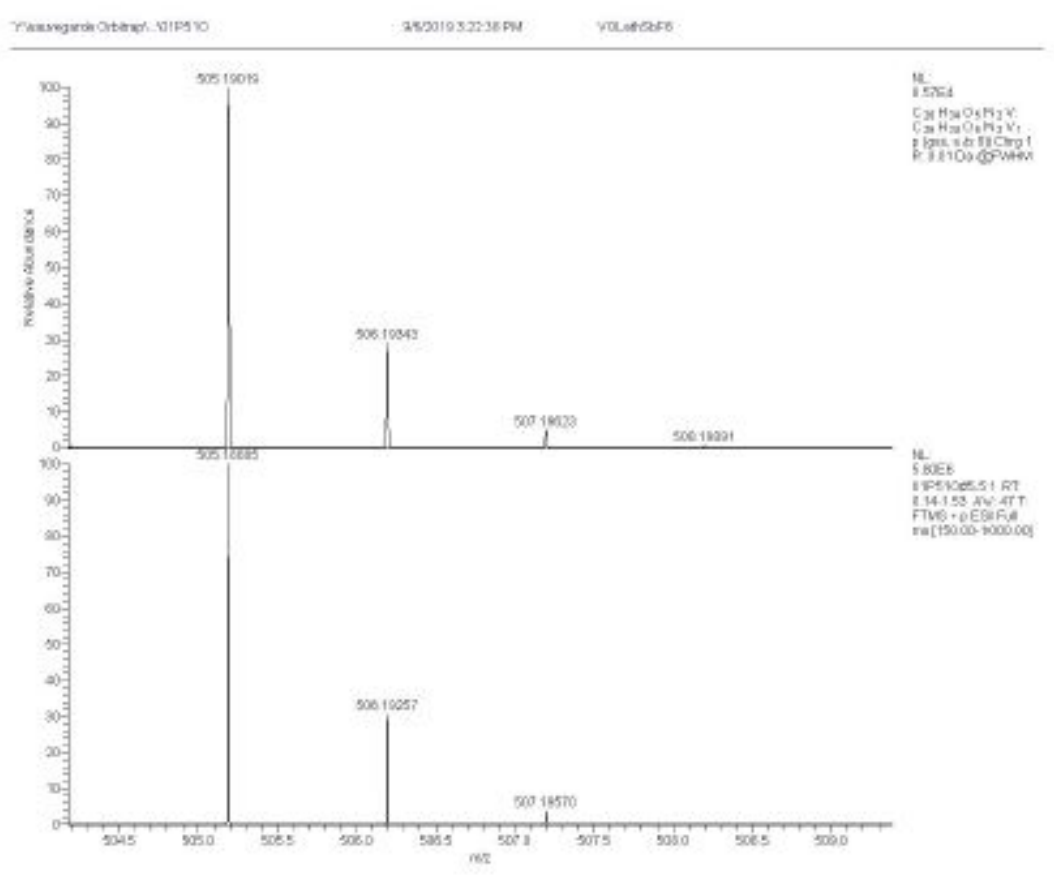

Fig. $\mathbf{S 7}$ HRMS spectrum of $\mathbf{1}^{+}\left(\mathrm{SbF}_{6}{ }^{-}\right)$(top: calculated, bottom: experimental)

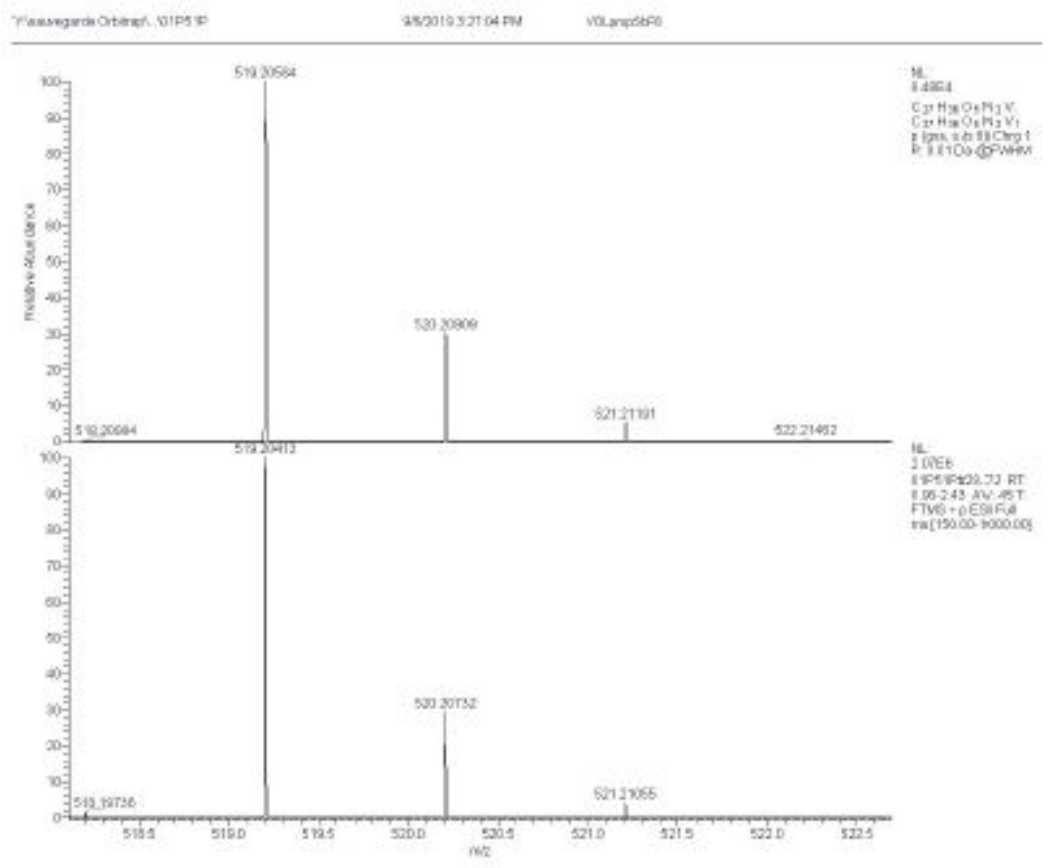

Fig. $\mathbf{S 8}$ HRMS spectrum of $\mathbf{2}^{+}\left(\mathbf{S b F}_{6}^{-}\right)$(top: calculated, bottom: experimental) 


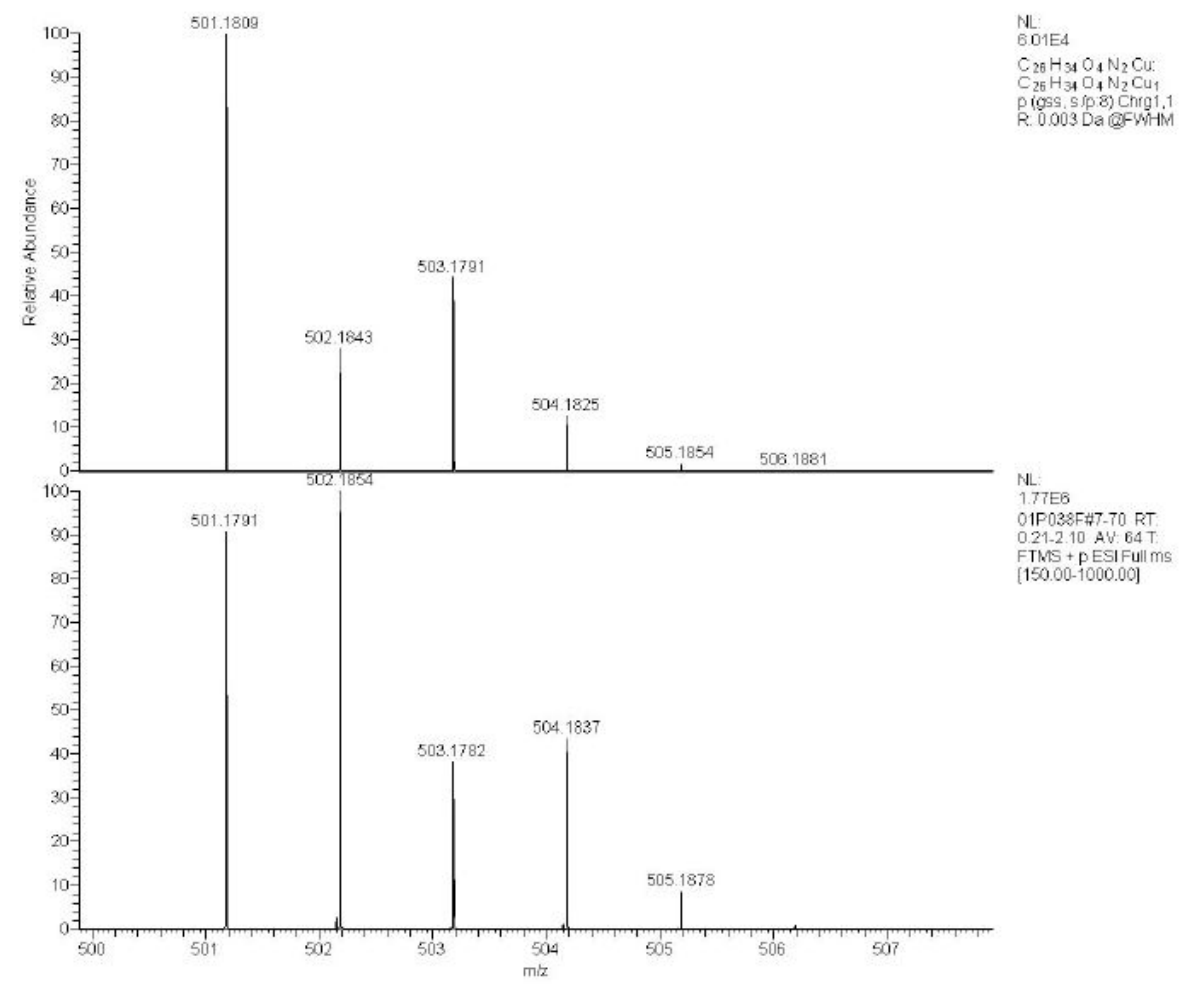

Fig. $\mathbf{S 9}$ HRMS spectrum of $\mathbf{3 , 3}^{+}\left(\mathrm{SbF}_{6}^{-}\right)$(top: calculated, bottom: experimental)

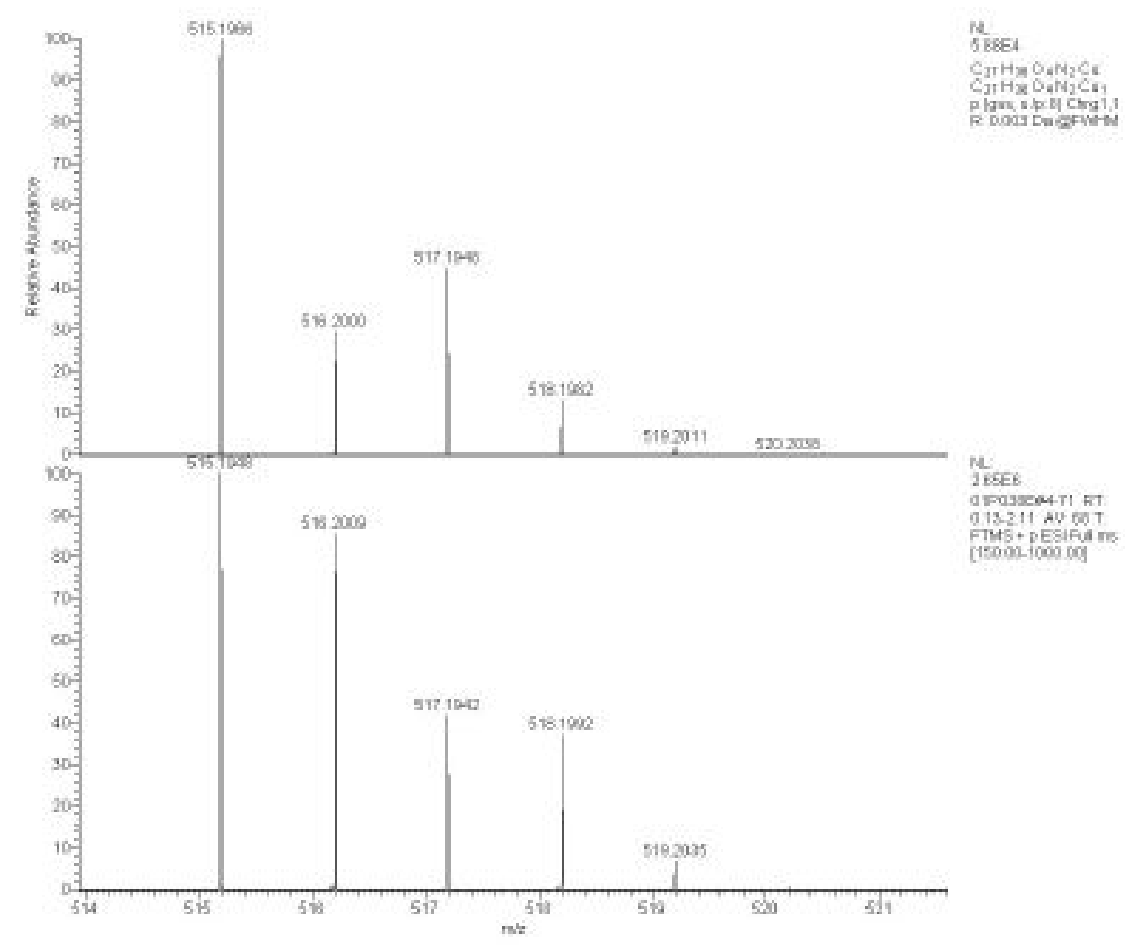

Fig. $\mathbf{S 1 0 ~ H R M S ~ s p e c t r u m ~ o f ~} \mathbf{4 , 4 ^ { + }}\left(\mathrm{SbF}_{6}{ }^{-}\right)$(top: calculated, bottom: experimental) 


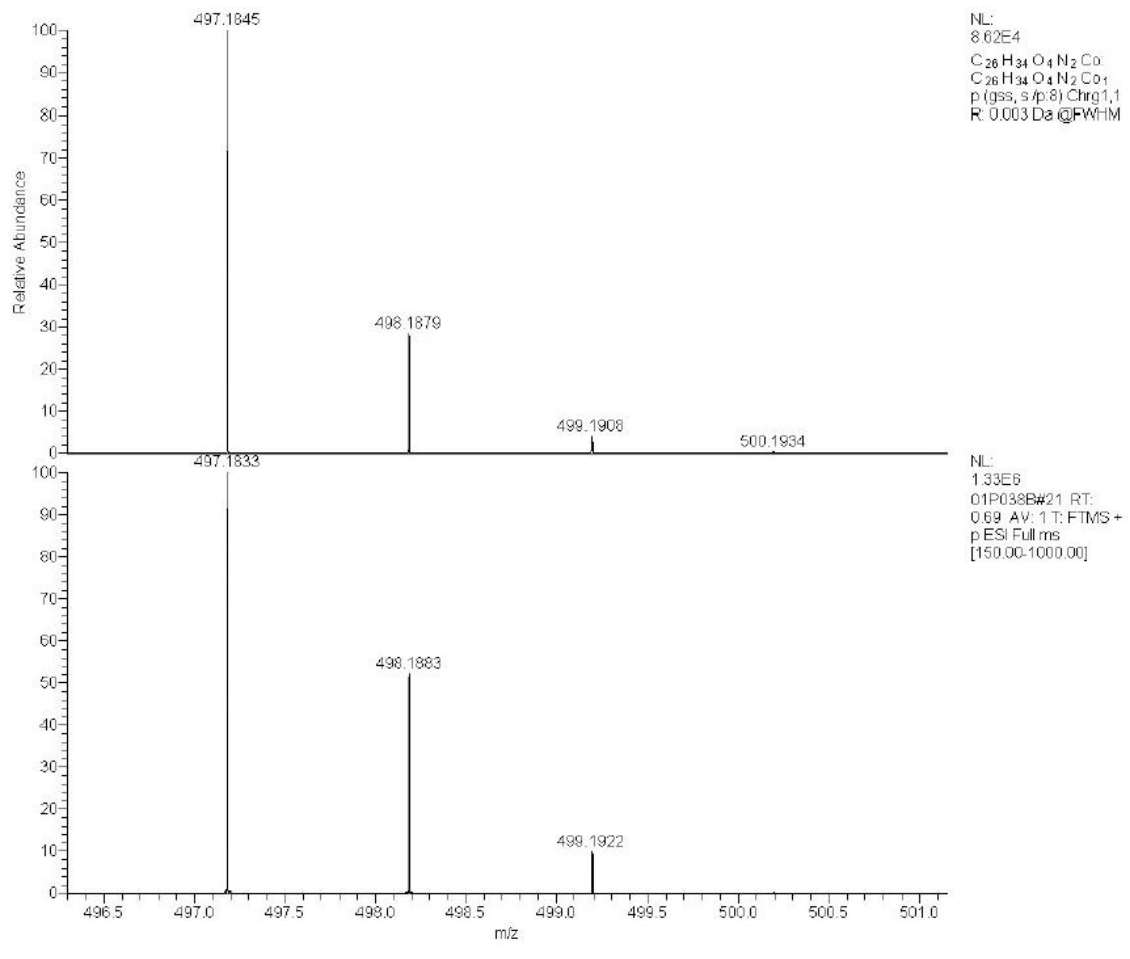

Fig. $\mathbf{S 1 1} \mathrm{HRMS}$ spectrum of $\mathbf{5}^{+}\left(\mathbf{S b F}_{6}{ }^{-}\right)$(top: calculated, bottom: experimental)

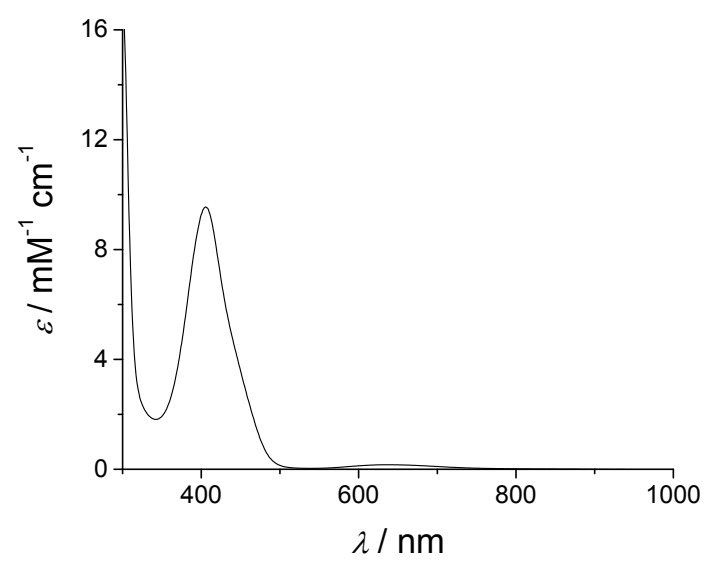

Fig. S12 UV-Vis spectrum of 1 in $\mathrm{CH}_{2} \mathrm{Cl}_{2}$ 


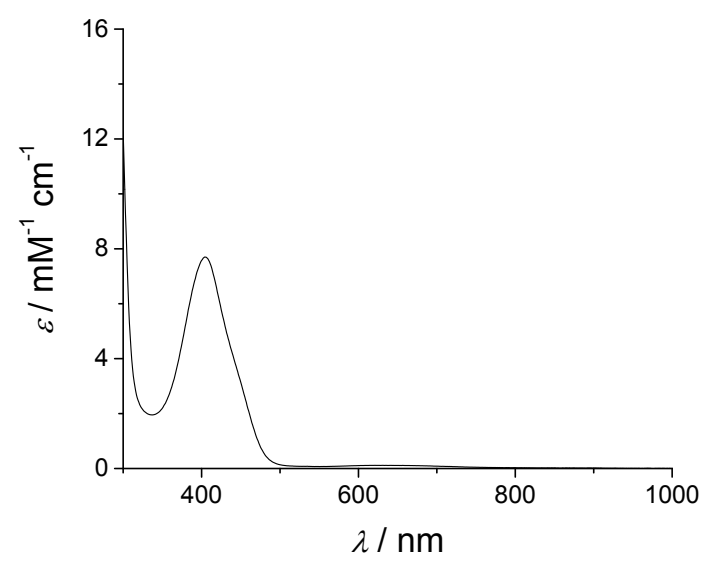

Fig. $\mathbf{S 1 3} \mathrm{UV}$-Vis spectrum of $\mathbf{2}$ in $\mathrm{CH}_{2} \mathrm{Cl}_{2}$

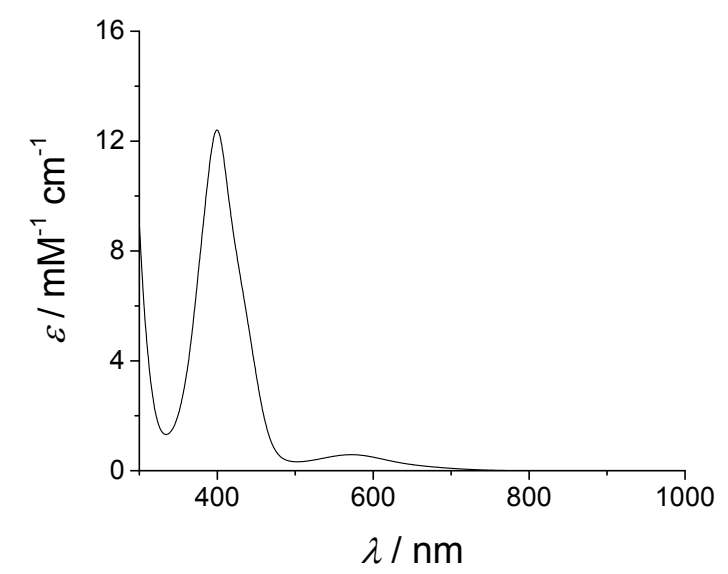

Fig. S14 UV-Vis spectrum of $\mathbf{3}$ in $\mathrm{CH}_{2} \mathrm{Cl}_{2}$

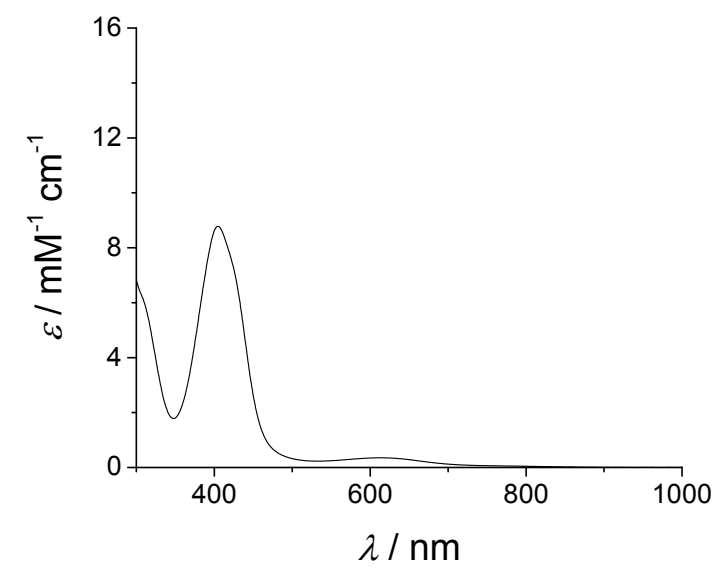

Fig. S15 UV-Vis spectrum of 4 in $\mathrm{CH}_{2} \mathrm{Cl}_{2}$ 


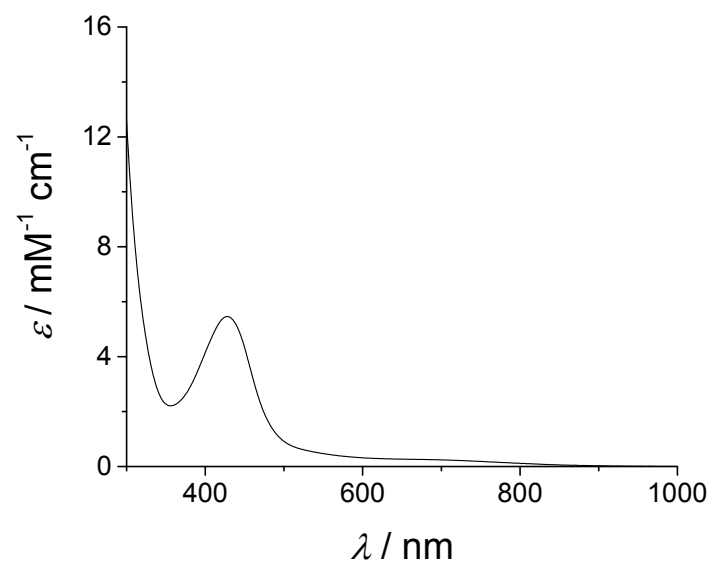

Fig. S16 UV-Vis spectrum of 6 in $\mathrm{CH}_{2} \mathrm{Cl}_{2}$

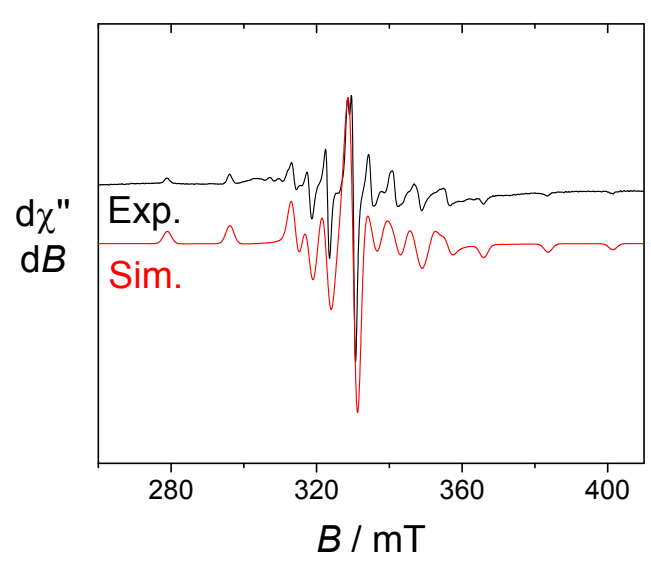

Fig. $\mathbf{S 1 7} \mathrm{X}$-Band EPR spectrum of a $1 \mathrm{mM} \mathrm{CH}_{2} \mathrm{Cl}_{2}$ solution of 2. Microwave power, $5 \mathrm{~mW}$; Freq. 9.33 GHz; Mod. Amp. 0.3 mT; Freq. 100 KHz. T = 100 K. Black line, experimental spectrum; Red line, simulated spectrum.

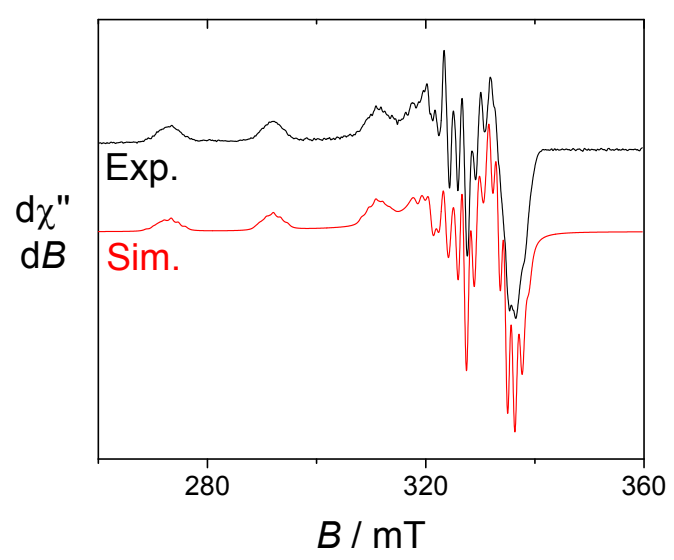

Fig. S18 X-Band EPR spectrum of a $1 \mathrm{mM} \mathrm{CH}_{2} \mathrm{Cl}_{2}$ solution of 4. Microwave power, $5 \mathrm{~mW}$; Freq. 9.33 GHz; Mod. Amp. 0.3 mT; Freq. 100 KHz. T=100 K. Black line, experimental spectrum; Red line, simulated spectrum. 


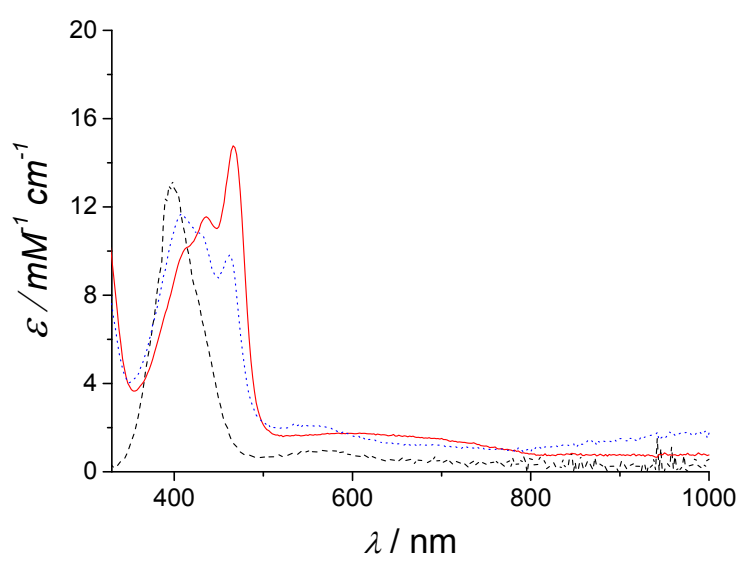

Fig. S19 UV-Vis spectra of 3 in $\mathrm{CH}_{2} \mathrm{Cl}_{2}$ containing $0.1 \mathrm{M}$ TBAP at various stages of electrolysis $(T=233$ K). Black curve: Initial; Blue curve: after removal of one-electron ( $\left.E_{a p p}=0.3 \mathrm{~V}\right)$; Red curve: after removal of two electrons $\left(E_{a p p}=0.5 \mathrm{~V}\right)$.

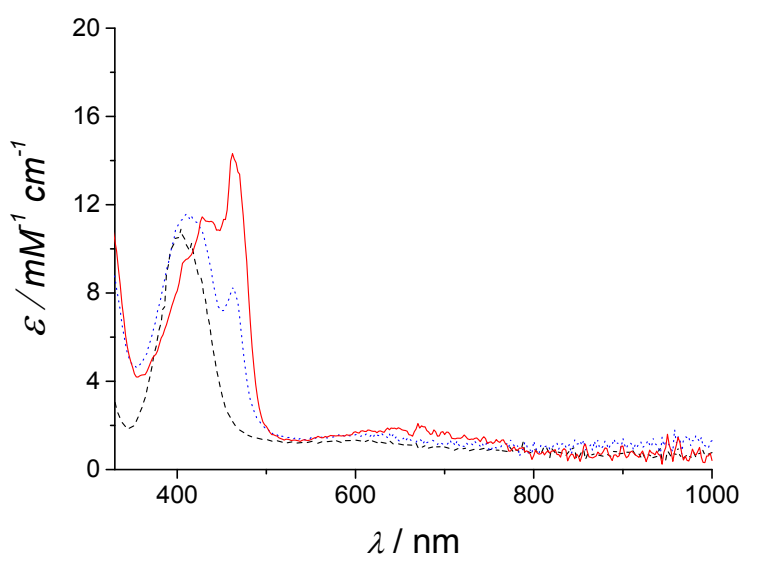

Fig. S2O UV-Vis spectra of 4 in $\mathrm{CH}_{2} \mathrm{Cl}_{2}$ containing $0.1 \mathrm{M}$ TBAP at various stages of electrolysis $(T=233$ $\mathrm{K})$. Black curve: Initial; Blue curve: after removal of one-electron ( $\left.\mathrm{E}_{\mathrm{app}}=0.3 \mathrm{~V}\right)$; Red curve: after removal of two electrons $\left(E_{a p p}=0.5 \mathrm{~V}\right)$.
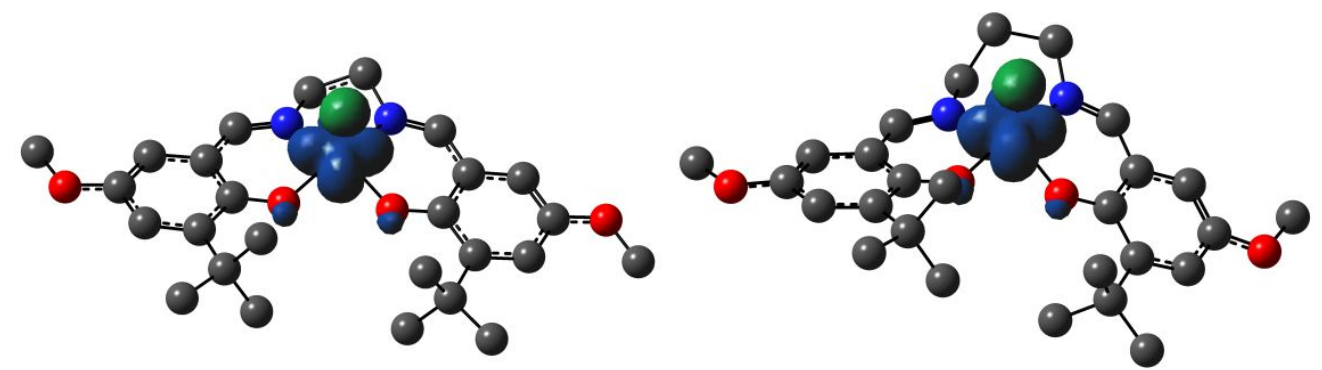

Fig. S21 Spin density plots for $\mathbf{1}$ and $\mathbf{2}$. See the Experimental Section for calculation details. 

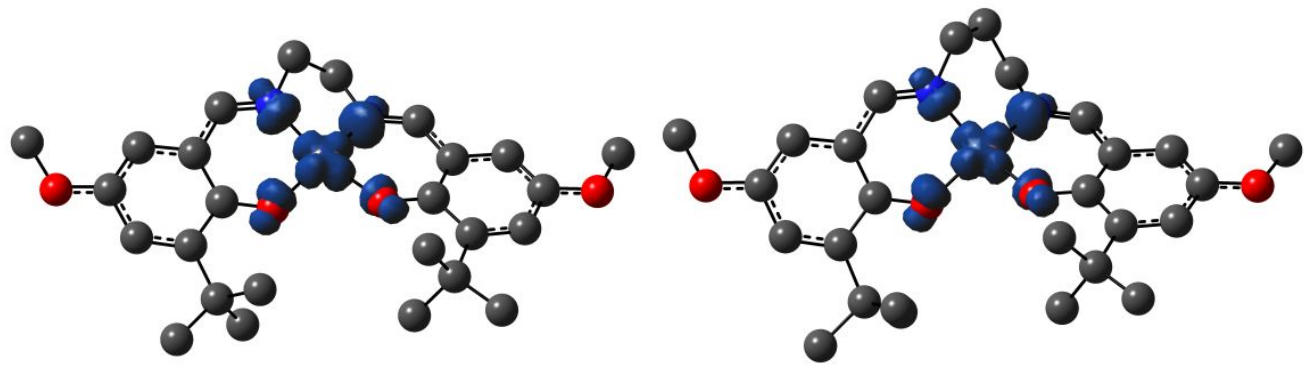

Fig. S22 Spin density plots for $\mathbf{3}$ and $\mathbf{4}$. See the Experimental Section for calculation details.

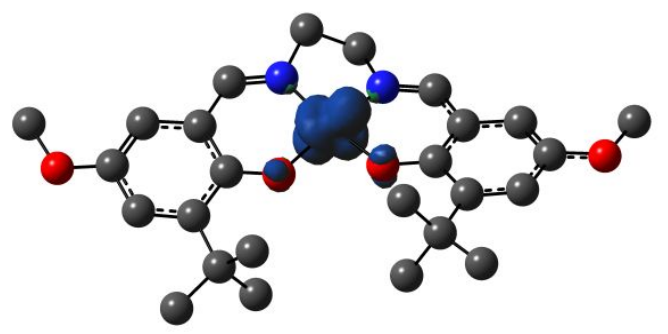

Fig. S23 Spin density plot for $5(S=1 / 2)$. See the Experimental Section for calculation details.

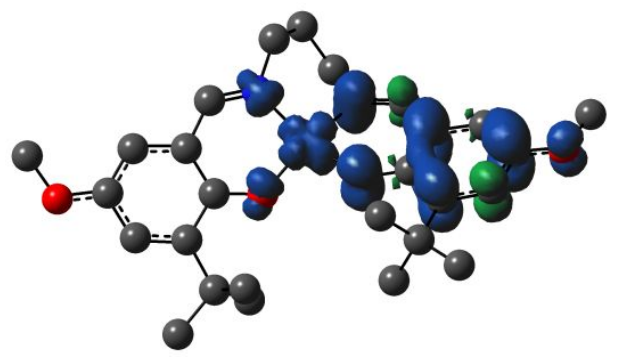

Fig. S24 Spin density plot for $\mathbf{4}^{+}$. See the Experimental Section for calculation details. 

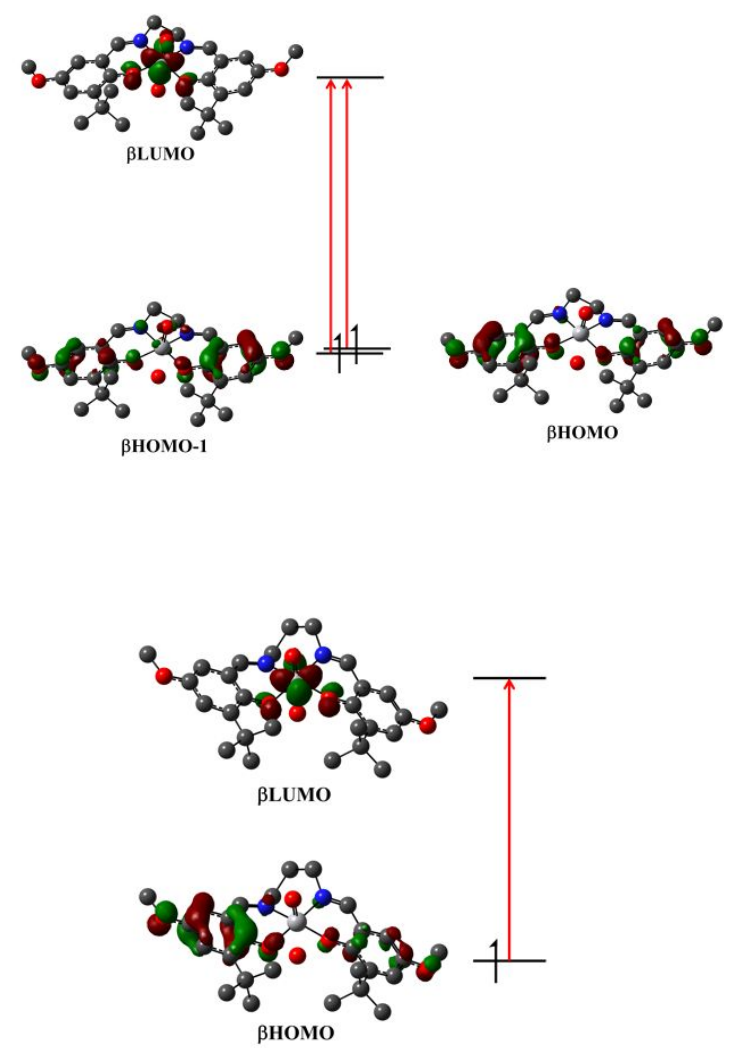

Fig. S25 Orbitals involved in the transition for $\mathbf{1}^{+}$(top, $f=0.0401, \lambda_{\max }=713 \mathrm{~nm}$ ) and $\mathbf{2}^{+}$(bottom, $f=$ $0.0587, \lambda_{\max }=760 \mathrm{~nm}$ as determined by TD-DFT calculations.
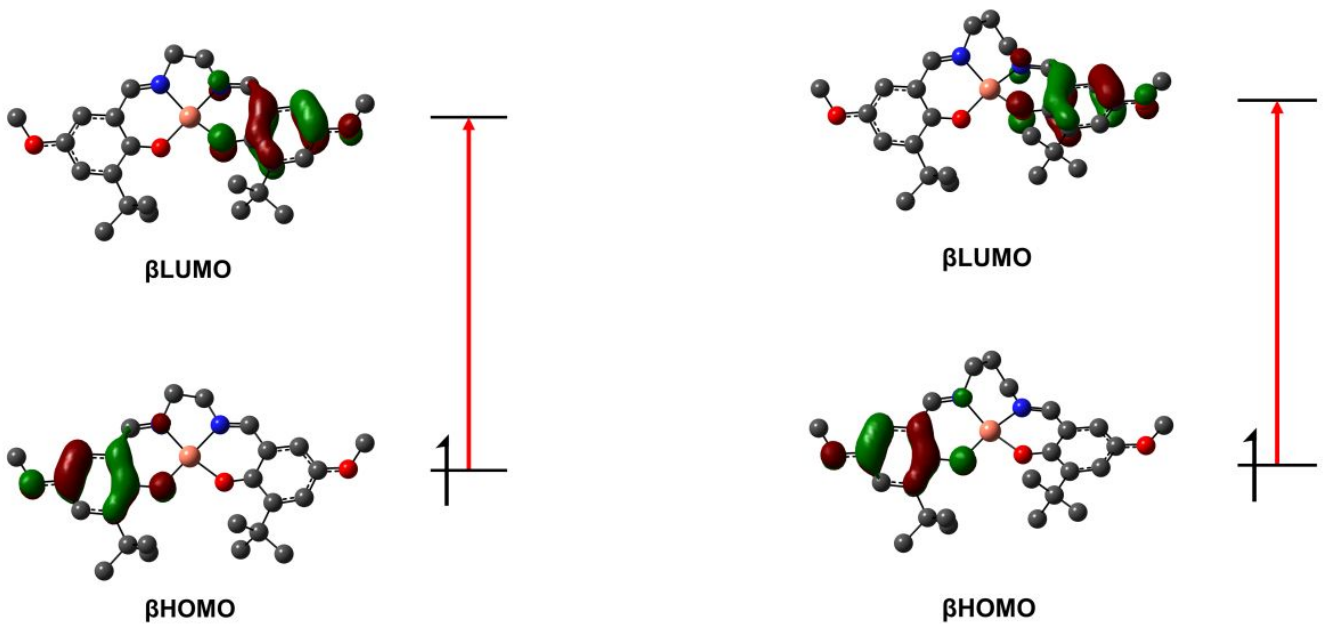

Fig. S26 Orbitals involved for the transitions for $\mathbf{3}^{+}$(left, $f=0.0585, \lambda_{\max }=1251 \mathrm{~nm}$ ) and $\mathbf{4}^{+}$(right, $f=$ $0.0326, \lambda_{\max }=1240 \mathrm{~nm}$ ) as determined by TD-DFT calculations. 


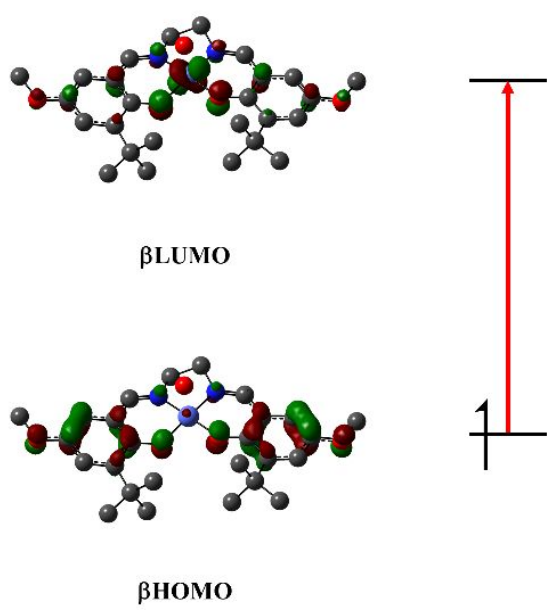

Fig. S27 Orbitals involved for the transitions for $\mathbf{5}^{+}\left(f=0.1179, \lambda_{\max }=1091 \mathrm{~nm}\right)$

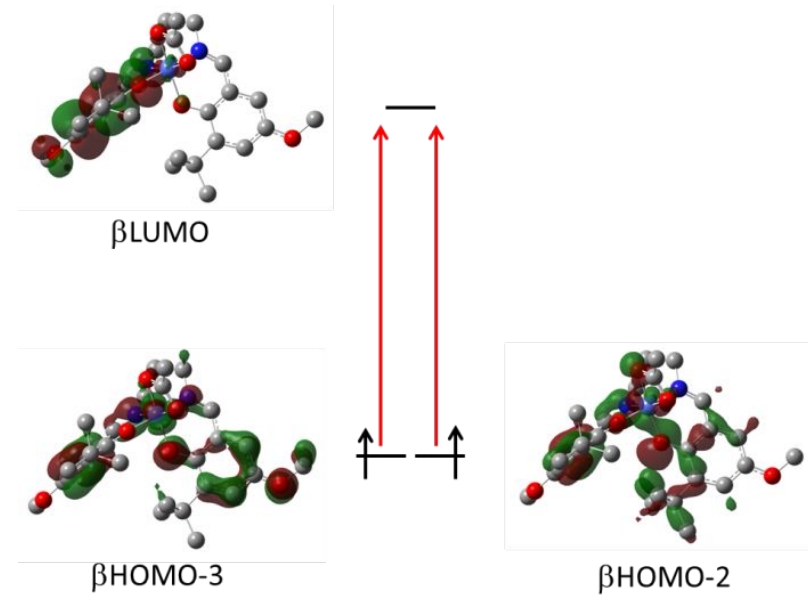

Fig. S28 Orbitals involved in the low energy transition for $\mathbf{6}^{+}\left(f=0.0156, \lambda_{\max }=656 \mathrm{~nm}\right)$ 


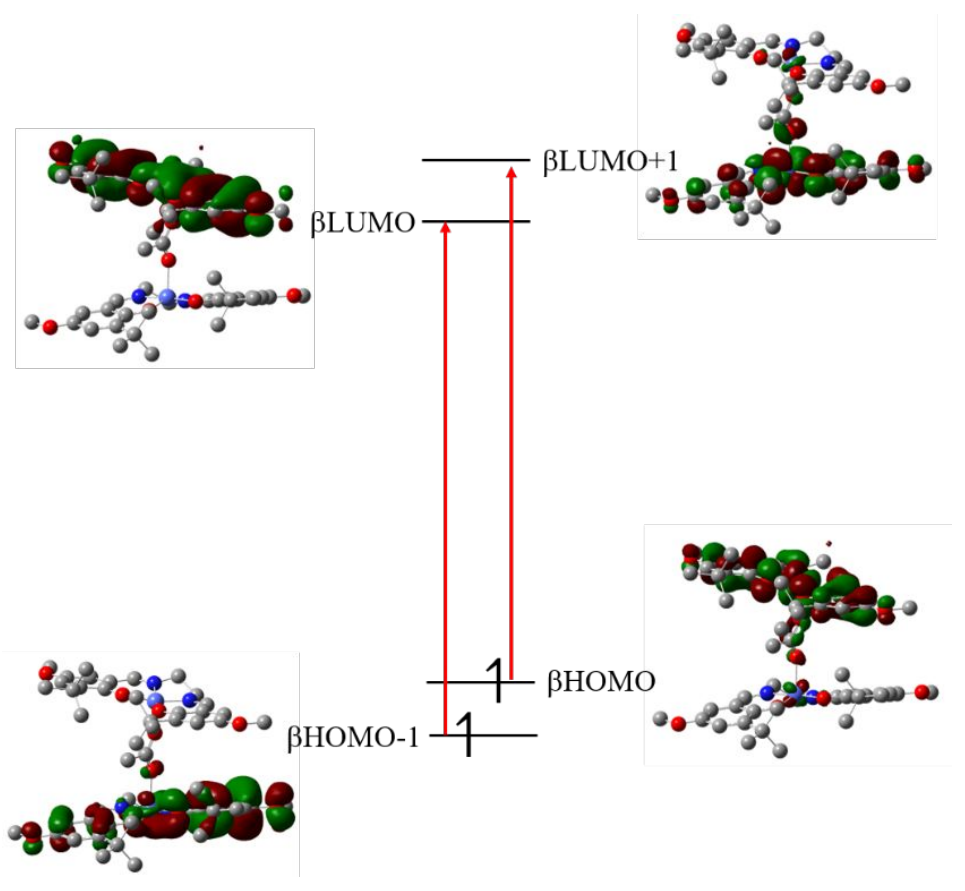

Figure S29. Predicted TD-DFT transitions for $\left(5^{+}\right)_{2}\left(\mu-O A c^{-}\right)$in the high-spin $(S=2)$ form. The two predicted transitions at $940 \mathrm{~nm}(f=0.1504)$ and $878 \mathrm{~nm}(f=0.1420)$ are the $\beta \mathrm{HOMO} \rightarrow \beta \mathrm{LUMO}+1$ and $\beta \mathrm{HOMO}-1 \rightarrow \beta \mathrm{LUMO}$ transitions respectively. Both transitions are primarily ligand-based, and predict the transfer of electron density between oxidized Co salen units. (See experimental section for calculation details) 


\section{Table}

Table S1. Experimental and calculated (in parentheses) coordination sphere metrical parameters for the complexes $[\AA]$.

\begin{tabular}{|l|l|l|l|l|l|l|}
\hline & \multicolumn{1}{|c|}{ M-01 } & \multicolumn{1}{|c|}{ M-02 } & \multicolumn{1}{|c|}{ M-N1 } & \multicolumn{1}{|c|}{ M-N2 } & \multicolumn{1}{c|}{ M-05 } & \multicolumn{1}{c|}{ M-06 } \\
\hline $\mathbf{1}$ & $1.921(1.950)$ & $1.913(1.935)$ & $2.055(2.083)$ & $2.043(2.080)$ & $1.595(1.581)$ & - \\
\hline $\mathbf{1}^{+}$ & $1.822(1.833)$ & $1.804(1.805)$ & $2.068(2.077)$ & $2.084(2.107)$ & $1.584(1.563)$ & $2.316(2.475)$ \\
\hline $\mathbf{2}$ & $1.911(1.934)$ & $1.942(1.965)$ & $2.098(2.094)$ & $2.065(2.092)$ & $1.592(1.581)$ & - \\
& 1.911 & 1.938 & 2.095 & 2.067 & 1.594 & \\
\hline $\mathbf{2}^{+}$ & $1.822(1.816)$ & $1.836(1.846)$ & $2.093(2.121)$ & $2.062(2.089)$ & $1.591(1.562)$ & $2.329(2.448)$ \\
\hline $\mathbf{3}$ & $1.894(1.901)$ & $1.892(1.901)$ & $1.935(1.929)$ & $1.913(1.929)$ & - & - \\
\hline $\mathbf{3}^{+}$ & $1.883(1.835)$ & $1.929(1.954)$ & $1.895(1.910)$ & $1.96(1.977)$ & - & - \\
\hline $\mathbf{4}$ & $1.883(1.897)$ & $1.900(1.897)$ & $1.949(1.936)$ & $1.933(1.936)$ & - & - \\
\hline $\mathbf{4}^{+}$ & $1.882(1.834)$ & $1.958(1.964)$ & $1.937(1.920)$ & $1.974(1.984)$ & - & - \\
\hline $\mathbf{5}$ & $1.847(1.855)$ & $1.854(1.855)$ & $1.854(1.856)$ & $1.845(1.856)$ & - & - \\
\hline $\mathbf{5}^{+}$ & $1.867(1.858)$ & $1.850(1.867)$ & $1.867(1.874)$ & $1.877(1.877)$ & $2.115(2.169)$ & - \\
\hline $\mathbf{6}$ & $1.870(1.888)$ & $1.855(1.876)$ & $1.883(1.909)$ & $1.889(1.914)$ & $1.995(1.979)$ & $1.997(1.977)$ \\
\hline
\end{tabular}

Table S2. Coordination sphere metrical parameters for the dimer $\left(5^{+}\right)_{2}(\mu-\mathrm{OAc})$ by X-ray crystallography and theoretical calculations in brackets. Values are reported in Angstroms.

\begin{tabular}{|l|l|l|l|l|}
\hline Co-O(Sal) & Co-O(Sal) & Co-N & Co-N & Co-O(OAc) \\
\hline $1.837(1.856)$ & $1.850(1.860)$ & $1.868(1.896)$ & $1.857(1.880)$ & $2.083(2.065)$ \\
\hline Co-O(Sal) & Co-O(Sal) & Co-N & Co-N & Co-O(OAc) \\
\hline $1.823(1.853)$ & $1.844(1.852)$ & $1.872(1.892)$ & $1.868(1.888)$ & $2.052(2.030)$ \\
\hline
\end{tabular}




\section{Computational Details}

\section{III.1 Optimized XYZ Coordinates}

A) Optimized XYZ Coordinates ( $(̊)$ for $\mathbf{1}$

\begin{tabular}{|c|c|c|c|}
\hline V & 0.069774 & -1.12403 & 0.653623 \\
\hline 0 & -1.38219 & -0.00917 & 0.026239 \\
\hline 0 & 1.37434 & 0.192724 & 0.036915 \\
\hline 0 & -6.85025 & -0.78533 & -0.36145 \\
\hline 0 & 6.862377 & 0.056296 & -0.52534 \\
\hline 0 & 0.191908 & -1.17084 & 2.228784 \\
\hline$N$ & -1.21148 & -2.71344 & 0.255095 \\
\hline $\mathrm{N}$ & 1.352381 & -2.53115 & -0.19049 \\
\hline C & -2.68845 & -0.16986 & -0.03971 \\
\hline C & -3.5518 & 0.966672 & -0.16698 \\
\hline C & -4.92506 & 0.744182 & -0.26595 \\
\hline C & -5.50333 & -0.54047 & -0.26164 \\
\hline C & -4.67949 & -1.64143 & -0.15339 \\
\hline C & -3.28327 & -1.47322 & -0.03284 \\
\hline C & -7.72889 & 0.318266 & -0.48774 \\
\hline C & -2.97812 & 2.397755 & -0.20894 \\
\hline C & -2.16805 & 2.687531 & 1.078315 \\
\hline C & -2.07164 & 2.548677 & -1.45467 \\
\hline C & -4.08295 & 3.469546 & -0.30647 \\
\hline C & -2.49095 & -2.66452 & 0.05744 \\
\hline C & 2.677898 & 0.124561 & -0.06613 \\
\hline C & 3.47728 & 1.32455 & 0.005475 \\
\hline C & 4.845579 & 1.19772 & -0.15456 \\
\hline C & 5.501296 & -0.0338 & -0.38989 \\
\hline C & 4.758201 & -1.18702 & -0.46142 \\
\hline C & 3.345917 & -1.12233 & -0.2895 \\
\hline C & 7.57623 & -1.14306 & -0.75903 \\
\hline C & 2.829166 & 2.697228 & 0.269092 \\
\hline C & 3.873689 & 3.83063 & 0.321558 \\
\hline C & 1.833346 & 3.03444 & -0.86628 \\
\hline C & 2.095514 & 2.675813 & 1.632454 \\
\hline C & 2.623356 & -2.34513 & -0.41328 \\
\hline C & -0.5112 & -3.99411 & 0.324919 \\
\hline C & 0.768851 & -3.84369 & -0.49901 \\
\hline $\mathrm{H}$ & -5.58046 & 1.599677 & -0.35284 \\
\hline $\mathrm{H}$ & -5.11596 & -2.63641 & -0.15212 \\
\hline $\mathrm{H}$ & -7.67971 & 0.982968 & 0.385717 \\
\hline $\mathrm{H}$ & -8.73276 & -0.10577 & -0.55692 \\
\hline $\mathrm{H}$ & -7.52297 & 0.903421 & -1.39468 \\
\hline $\mathrm{H}$ & -1.32961 & 1.999408 & 1.186538 \\
\hline $\mathrm{H}$ & -2.80653 & 2.599923 & 1.965798 \\
\hline $\mathrm{H}$ & -1.7743 & 3.711117 & 1.047592 \\
\hline $\mathrm{H}$ & -1.25486 & 1.82576 & -1.43738 \\
\hline
\end{tabular}




$\begin{array}{lrrr}\text { H } & -2.65066 & 2.399745 & -2.37451 \\ \text { H } & -1.64164 & 3.55739 & -1.4871 \\ \text { H } & -4.67867 & 3.372498 & -1.22213 \\ \text { H } & -4.76235 & 3.444365 & 0.554114 \\ \text { H } & -3.61755 & 4.461183 & -0.32586 \\ \text { H } & -3.03234 & -3.61101 & -0.05488 \\ \text { H } & 5.488382 & 2.06814 & -0.10291 \\ \text { H } & 5.21713 & -2.15417 & -0.6383 \\ \text { H } & 7.263319 & -1.62402 & -1.69662 \\ \text { H } & 7.449989 & -1.85655 & 0.067468 \\ \text { H } & 8.628266 & -0.8609 & -0.83335 \\ \text { H } & 4.417577 & 3.937314 & -0.6245 \\ \text { H } & 3.36151 & 4.779945 & 0.513763 \\ \text { H } & 4.60497 & 3.684692 & 1.12532 \\ \text { H } & 2.349234 & 3.080767 & -1.83336 \\ \text { H } & 1.039145 & 2.290447 & -0.93097 \\ \text { H } & 1.376641 & 4.014974 & -0.68232 \\ \text { H } & 2.802662 & 2.489563 & 2.449679 \\ \text { H } & 1.325908 & 1.903501 & 1.662353 \\ \text { H } & 1.619233 & 3.647231 & 1.814497 \\ \text { H } & 3.210565 & -3.21127 & -0.74104 \\ \text { H } & -1.12783 & -4.82367 & -0.04533 \\ \text { H } & -0.25647 & -4.1905 & 1.37431 \\ \text { H } & 0.515738 & -3.8797 & -1.5678 \\ \text { H } & 1.474982 & -4.6582 & -0.28994\end{array}$

B) Optimized XYZ Coordinates $(\AA)$ for $\mathbf{2}$

$\begin{array}{lrrr}\text { V } & 0.028825 & 1.091878 & 0.752765 \\ \mathrm{O} & 1.34495 & -0.19922 & 0.167148 \\ \mathrm{O} & -1.32751 & -0.24142 & 0.256731 \\ \mathrm{O} & 6.77614 & -0.41842 & -0.79496 \\ \mathrm{O} & -6.65035 & 0.005153 & -1.16285 \\ \mathrm{O} & -0.23286 & 1.296792 & 2.2987 \\ \mathrm{~N} & 1.60341 & 2.498622 & 0.618751 \\ \mathrm{~N} & -1.09738 & 2.430847 & -0.39437 \\ \mathrm{C} & 2.642514 & -0.22779 & -0.04214 \\ \mathrm{C} & 3.302022 & -1.46776 & -0.36105 \\ \mathrm{C} & 4.667616 & -1.43025 & -0.59198 \\ \mathrm{C} & 5.440618 & -0.24938 & -0.53639 \\ \mathrm{C} & 4.82253 & 0.943005 & -0.23774 \\ \mathrm{C} & 3.423274 & 0.961376 & 0.012594 \\ \mathrm{C} & 7.601275 & 0.730391 & -0.74668 \\ \mathrm{C} & 2.514928 & -2.78765 & -0.45849 \\ \mathrm{C} & 1.467861 & -2.67513 & -1.59339 \\ \mathrm{C} & 1.821332 & -3.08905 & 0.892158 \\ \mathrm{C} & 3.42839 & -3.98615 & -0.78658 \\ \mathrm{C} & 2.844551 & 2.2268 & 0.341308 \\ \mathrm{C} & -2.59846 & -0.1433 & -0.02764\end{array}$




\begin{tabular}{|c|c|c|c|}
\hline C & -3.50064 & -1.24735 & 0.204953 \\
\hline C & -4.81443 & -1.09994 & -0.20286 \\
\hline C & -5.32324 & 0.066262 & -0.82218 \\
\hline C & -4.48966 & 1.140813 & -1.01519 \\
\hline C & -3.12907 & 1.052393 & -0.60264 \\
\hline C & -7.21979 & 1.147944 & -1.77238 \\
\hline C & -3.02381 & -2.53388 & 0.904263 \\
\hline C & -1.94196 & -3.22497 & 0.043302 \\
\hline C & -2.45245 & -2.18682 & 2.301486 \\
\hline C & -4.17151 & -3.54286 & 1.110577 \\
\hline C & -2.32717 & 2.215132 & -0.7819 \\
\hline C & 1.335015 & 3.873025 & 1.074776 \\
\hline C & -0.045 & 4.402223 & 0.642334 \\
\hline C & -0.5511 & 3.770859 & -0.65854 \\
\hline $\mathrm{H}$ & 5.20936 & -2.33688 & -0.83251 \\
\hline $\mathrm{H}$ & 5.37458 & 1.875392 & -0.18047 \\
\hline $\mathrm{H}$ & 8.612166 & 0.388219 & -0.97674 \\
\hline $\mathrm{H}$ & 7.294927 & 1.480178 & -1.48983 \\
\hline $\mathrm{H}$ & 7.593451 & 1.192532 & 0.250516 \\
\hline $\mathrm{H}$ & 1.963318 & -2.50518 & -2.55732 \\
\hline $\mathrm{H}$ & 0.770535 & -1.85599 & -1.41127 \\
\hline $\mathrm{H}$ & 0.895317 & -3.60742 & -1.66996 \\
\hline $\mathrm{H}$ & 1.124348 & -2.29775 & 1.169793 \\
\hline $\mathrm{H}$ & 1.26508 & -4.0318 & 0.823776 \\
\hline $\mathrm{H}$ & 2.565178 & -3.19503 & 1.691279 \\
\hline $\mathrm{H}$ & 3.931575 & -3.87245 & -1.75402 \\
\hline $\mathrm{H}$ & 2.8196 & -4.89551 & -0.83994 \\
\hline $\mathrm{H}$ & 4.192763 & -4.14719 & -0.01715 \\
\hline $\mathrm{H}$ & 3.557213 & 3.059703 & 0.377879 \\
\hline $\mathrm{H}$ & -5.52966 & -1.89962 & -0.05201 \\
\hline $\mathrm{H}$ & -4.8403 & 2.065468 & -1.4617 \\
\hline $\mathrm{H}$ & -6.72268 & 1.389779 & -2.7225 \\
\hline $\mathrm{H}$ & -7.17197 & 2.025357 & -1.11184 \\
\hline $\mathrm{H}$ & -8.26536 & 0.900363 & -1.96607 \\
\hline $\mathrm{H}$ & -1.0854 & -2.56975 & -0.11085 \\
\hline $\mathrm{H}$ & -2.34731 & -3.50704 & -0.93637 \\
\hline $\mathrm{H}$ & -1.59584 & -4.13982 & 0.540608 \\
\hline $\mathrm{H}$ & -1.62596 & -1.47774 & 2.236123 \\
\hline $\mathrm{H}$ & -3.2308 & -1.7496 & 2.938591 \\
\hline $\mathrm{H}$ & -2.09023 & -3.09869 & 2.79258 \\
\hline $\mathrm{H}$ & -4.60296 & -3.88031 & 0.160733 \\
\hline $\mathrm{H}$ & -3.78031 & -4.42774 & 1.624757 \\
\hline $\mathrm{H}$ & -4.9778 & -3.13266 & 1.729889 \\
\hline $\mathrm{H}$ & -2.83042 & 3.041712 & -1.29633 \\
\hline $\mathrm{H}$ & 2.122849 & 4.535898 & 0.690743 \\
\hline $\mathrm{H}$ & 1.401043 & 3.883788 & 2.169419 \\
\hline $\mathrm{H}$ & 0.024541 & 5.489348 & 0.517951 \\
\hline $\mathrm{H}$ & -0.78964 & 4.216608 & 1.423303 \\
\hline $\mathrm{H}$ & 0.265725 & 3.688287 & -1.38718 \\
\hline
\end{tabular}


C) Optimized XYZ Coordinates ( $\AA$ ) for $\mathbf{3}$

\begin{tabular}{|c|c|c|c|}
\hline $\mathrm{Cu}$ & $-4 \mathrm{E}-05$ & -1.09795 & $-7.3 \mathrm{E}-05$ \\
\hline 0 & -1.38002 & 0.2019 & 0.140535 \\
\hline $\mathrm{O}$ & 1.379979 & 0.201861 & -0.14067 \\
\hline 0 & -6.88691 & -0.21636 & -0.1617 \\
\hline 0 & 6.886846 & -0.2165 & 0.161999 \\
\hline$N$ & -1.25418 & -2.51837 & -0.36319 \\
\hline $\mathrm{N}$ & 1.254061 & -2.5184 & 0.363039 \\
\hline C & -2.67602 & 0.055063 & 0.052603 \\
\hline C & -3.53924 & 1.205644 & 0.226601 \\
\hline C & -4.90794 & 1.018764 & 0.137581 \\
\hline C & -5.51386 & -0.23355 & -0.11245 \\
\hline C & -4.71579 & -1.34032 & -0.28098 \\
\hline C & -3.3002 & -1.21202 & -0.20429 \\
\hline C & -7.54605 & -1.45114 & -0.40191 \\
\hline C & -2.94907 & 2.604075 & 0.503102 \\
\hline C & -4.04356 & 3.680357 & 0.655065 \\
\hline C & -2.13796 & 2.583315 & 1.821805 \\
\hline C & -2.04088 & 3.033181 & -0.67515 \\
\hline C & -2.54707 & -2.41995 & -0.37097 \\
\hline C & 2.675972 & 0.055015 & -0.05265 \\
\hline C & 3.539246 & 1.205574 & -0.2266 \\
\hline C & 4.907925 & 1.018663 & -0.13745 \\
\hline C & 5.513804 & -0.23366 & 0.112633 \\
\hline C & 4.715687 & -1.34042 & 0.281082 \\
\hline C & 3.300113 & -1.21208 & 0.204289 \\
\hline C & 7.545934 & -1.4513 & 0.40226 \\
\hline C & 2.949219 & 2.604054 & -0.50315 \\
\hline C & 2.138323 & 2.583454 & -1.82199 \\
\hline C & 2.040934 & 3.033259 & 0.675005 \\
\hline C & 4.043886 & 3.680178 & -0.65492 \\
\hline C & 2.546948 & -2.42 & 0.370906 \\
\hline C & -0.59392 & -3.81882 & -0.48716 \\
\hline C & 0.593777 & -3.81884 & 0.486967 \\
\hline $\mathrm{H}$ & -5.58558 & 1.855315 & 0.261676 \\
\hline $\mathrm{H}$ & -5.13567 & -2.32199 & -0.47342 \\
\hline $\mathrm{H}$ & -7.32407 & -2.18515 & 0.384175 \\
\hline $\mathrm{H}$ & -7.26548 & -1.87437 & -1.37557 \\
\hline $\mathrm{H}$ & -8.61501 & -1.2306 & -0.3987 \\
\hline $\mathrm{H}$ & -4.64847 & 3.786564 & -0.25295 \\
\hline $\mathrm{H}$ & -4.71676 & 3.469696 & 1.494208 \\
\hline $\mathrm{H}$ & -3.56887 & 4.64877 & 0.84947 \\
\hline $\mathrm{H}$ & -2.78799 & 2.334857 & 2.670005 \\
\hline $\mathrm{H}$ & -1.70346 & 3.572962 & 2.011171 \\
\hline $\mathrm{H}$ & -1.3308 & 1.851043 & 1.777215 \\
\hline $\mathrm{H}$ & .6 & 4.020569 & -0.4739 \\
\hline
\end{tabular}




$\begin{array}{lrrr}\text { H } & -1.2295 & 2.321049 & -0.82949 \\ \mathrm{H} & -2.62272 & 3.106006 & -1.60241 \\ \mathrm{H} & -3.1318 & -3.33581 & -0.51262 \\ \mathrm{H} & 5.585594 & 1.855209 & -0.26148 \\ \mathrm{H} & 5.135539 & -2.32209 & 0.473557 \\ \mathrm{H} & 7.32401 & -2.1853 & -0.38385 \\ \mathrm{H} & 7.265271 & -1.87453 & 1.375893 \\ \mathrm{H} & 8.6149 & -1.23078 & 0.399147 \\ \mathrm{H} & 2.788453 & 2.334965 & -2.67009 \\ \mathrm{H} & 1.33107 & 1.851278 & -1.77757 \\ \mathrm{H} & 1.703962 & 3.573163 & -2.01137 \\ \mathrm{H} & 1.22942 & 2.321255 & 0.829207 \\ \mathrm{H} & 1.606463 & 4.020725 & 0.473793 \\ \mathrm{H} & 2.622665 & 3.105958 & 1.602345 \\ \mathrm{H} & 3.569362 & 4.648696 & -0.8492 \\ \mathrm{H} & 4.648776 & 3.786144 & 0.253137 \\ \mathrm{H} & 4.717087 & 3.469526 & -1.49407 \\ \mathrm{H} & 3.131655 & -3.33588 & 0.512592 \\ \mathrm{H} & -1.27963 & -4.64803 & -0.27596 \\ \mathrm{H} & -0.22063 & -3.93063 & -1.51337 \\ \mathrm{H} & 1.279475 & -4.64806 & 0.275741 \\ \mathrm{H} & 0.220479 & -3.93068 & 1.513178\end{array}$

D) Optimized XYZ Coordinates $(\AA)$ for $\mathbf{4}$

$\begin{array}{lrrr}\mathrm{Cu} & -1.9 \mathrm{E}-05 & -0.97055 & -2.2 \mathrm{E}-05 \\ \mathrm{O} & -1.37429 & 0.322102 & 0.245517 \\ \mathrm{O} & 1.374276 & 0.322071 & -0.24557 \\ \mathrm{O} & -6.86464 & -0.04051 & -0.30432 \\ \mathrm{O} & 6.864613 & -0.04056 & 0.304423 \\ \mathrm{~N} & -1.21703 & -2.30102 & -0.7012 \\ \mathrm{~N} & 1.216977 & -2.30103 & 0.701174 \\ \mathrm{C} & -2.66689 & 0.190666 & 0.103145 \\ \mathrm{C} & -3.54305 & 1.287616 & 0.458477 \\ \mathrm{C} & -4.90717 & 1.117316 & 0.294725 \\ \mathrm{C} & -5.49385 & -0.065 & -0.21057 \\ \mathrm{C} & -4.68102 & -1.11727 & -0.56143 \\ \mathrm{C} & -3.27049 & -1.00717 & -0.40488 \\ \mathrm{C} & -7.50459 & -1.20053 & -0.81556 \\ \mathrm{C} & -2.97127 & 2.613354 & 1.001254 \\ \mathrm{C} & -2.16991 & 2.357297 & 2.301122 \\ \mathrm{C} & -2.05746 & 3.260826 & -0.06721 \\ \mathrm{C} & -4.07846 & 3.632368 & 1.339477 \\ \mathrm{C} & -2.50317 & -2.15409 & -0.79584 \\ \mathrm{C} & 2.666874 & 0.190646 & -0.10314 \\ \mathrm{C} & 3.543052 & 1.287589 & -0.45845 \\ \mathrm{C} & 4.90717 & 1.117272 & -0.29468 \\ \mathrm{C} & 5.493821 & -0.06505 & 0.210644 \\ \mathrm{C} & 4.680974 & -1.1173 & 0.561496\end{array}$




\begin{tabular}{|c|c|c|c|}
\hline$c$ & 3.270449 & -1.00719 & 0.404905 \\
\hline C & 7.504548 & -1.20061 & 0.815637 \\
\hline C & 2.971345 & 2.613337 & -1.00127 \\
\hline C & 4.078611 & 3.632285 & -1.33944 \\
\hline C & 2.17005 & 2.357314 & -2.30119 \\
\hline C & 2.057506 & 3.260874 & 0.067139 \\
\hline C & 2.503112 & -2.15411 & 0.795848 \\
\hline C & -0.59605 & -3.54695 & -1.15511 \\
\hline C & $-1.7 \mathrm{E}-05$ & -4.37917 & $-4.2 \mathrm{E}-05$ \\
\hline C & 0.595976 & -3.54696 & 1.155054 \\
\hline $\mathrm{H}$ & -5.59497 & 1.913589 & 0.554756 \\
\hline $\mathrm{H}$ & -5.08588 & -2.04313 & -0.95603 \\
\hline $\mathrm{H}$ & -7.3101 & -2.07879 & -0.18557 \\
\hline $\mathrm{H}$ & -7.18175 & -1.41883 & -1.84228 \\
\hline $\mathrm{H}$ & -8.57436 & -0.98381 & -0.812 \\
\hline $\mathrm{H}$ & -2.81928 & 1.935153 & 3.078197 \\
\hline $\mathrm{H}$ & -1.34341 & 1.666934 & 2.126964 \\
\hline $\mathrm{H}$ & -1.76235 & 3.302112 & 2.682315 \\
\hline $\mathrm{H}$ & -1.24022 & 2.593707 & -0.34418 \\
\hline $\mathrm{H}$ & -1.63041 & 4.195157 & 0.318786 \\
\hline $\mathrm{H}$ & -2.63247 & 3.502541 & -0.9697 \\
\hline $\mathrm{H}$ & -3.61595 & 4.550352 & 1.719326 \\
\hline $\mathrm{H}$ & -4.67381 & 3.905056 & 0.460269 \\
\hline $\mathrm{H}$ & -4.76008 & 3.261958 & 2.114285 \\
\hline $\mathrm{H}$ & -3.0774 & -2.98521 & -1.22114 \\
\hline $\mathrm{H}$ & 5.594979 & 1.913535 & -0.55471 \\
\hline $\mathrm{H}$ & 5.085821 & -2.04316 & 0.956113 \\
\hline $\mathrm{H}$ & 7.310022 & -2.07886 & 0.185632 \\
\hline $\mathrm{H}$ & 7.18172 & -1.41892 & 1.842352 \\
\hline $\mathrm{H}$ & 8.574318 & -0.98392 & 0.812063 \\
\hline $\mathrm{H}$ & 4.673836 & 3.905044 & -0.46017 \\
\hline $\mathrm{H}$ & 4.760334 & 3.261771 & -2.1141 \\
\hline $\mathrm{H}$ & 3.61618 & 4.550243 & -1.71945 \\
\hline $\mathrm{H}$ & 2.819441 & 1.935104 & -3.07821 \\
\hline $\mathrm{H}$ & 1.762597 & 3.302154 & -2.68243 \\
\hline $\mathrm{H}$ & 1.343487 & 1.667021 & -2.12708 \\
\hline $\mathrm{H}$ & 1.240231 & 2.593799 & 0.344084 \\
\hline $\mathrm{H}$ & 2.632488 & 3.502585 & 0.969651 \\
\hline $\mathrm{H}$ & 1.630517 & 4.195216 & -0.3189 \\
\hline $\mathrm{H}$ & 3.077329 & -2.98523 & 1.221152 \\
\hline $\mathrm{H}$ & -1.31798 & -4.15598 & -1.7138 \\
\hline $\mathrm{H}$ & 0.205226 & -3.27376 & -1.85037 \\
\hline $\mathrm{H}$ & -0.77078 & -5.03119 & 0.426762 \\
\hline $\mathrm{H}$ & 0.770771 & -5.03114 & -0.42686 \\
\hline $\mathrm{H}$ & -0.20532 & -3.27378 & 1.850289 \\
\hline $\mathrm{H}$ & 1.317889 & -4.156 & 1.71376 \\
\hline
\end{tabular}




\begin{tabular}{|c|c|c|c|}
\hline Co & $1.1 \mathrm{E}-06$ & -1.19147 & $-7.2 \mathrm{E}-05$ \\
\hline 0 & 1.306039 & 0.120754 & -0.11302 \\
\hline 0 & -1.30601 & 0.120785 & 0.112726 \\
\hline 0 & 6.822048 & -0.10868 & 0.173747 \\
\hline 0 & -6.822 & -0.10858 & -0.17451 \\
\hline $\mathrm{N}$ & 1.240199 & -2.54545 & 0.273054 \\
\hline $\mathrm{N}$ & -1.24019 & -2.54552 & -0.27285 \\
\hline C & 2.613305 & 0.024278 & -0.05046 \\
\hline C & 3.433186 & 1.201328 & -0.23805 \\
\hline C & 4.80685 & 1.058295 & -0.14468 \\
\hline C & 5.451984 & -0.17372 & 0.113999 \\
\hline C & 4.692597 & -1.30829 & 0.275457 \\
\hline C & 3.274473 & -1.22116 & 0.192045 \\
\hline C & 7.522737 & -1.31715 & 0.431725 \\
\hline C & 2.802231 & 2.576122 & -0.53603 \\
\hline C & 3.863453 & 3.682776 & -0.70217 \\
\hline C & 1.997502 & 2.505749 & -1.85693 \\
\hline C & 1.876264 & 2.99263 & 0.63163 \\
\hline C & 2.538302 & -2.43535 & 0.331524 \\
\hline C & -2.61327 & 0.024286 & 0.050259 \\
\hline C & -3.43317 & 1.201312 & 0.237951 \\
\hline $\mathrm{C}$ & -4.80683 & 1.058296 & 0.144467 \\
\hline C & -5.45194 & -0.17368 & -0.11445 \\
\hline$C$ & -4.69256 & -1.30824 & -0.27586 \\
\hline C & -3.27444 & -1.22116 & -0.19227 \\
\hline$C$ & -7.52271 & -1.31713 & -0.43198 \\
\hline$C$ & -2.80225 & 2.576066 & 0.53627 \\
\hline C & -1.99763 & 2.505313 & 1.857211 \\
\hline$C$ & -1.8762 & 2.992902 & -0.63121 \\
\hline$C$ & -3.86353 & 3.682627 & 0.702611 \\
\hline$C$ & -2.53829 & -2.4354 & -0.33138 \\
\hline$C$ & 0.620657 & -3.86792 & 0.442272 \\
\hline$C$ & -0.62073 & -3.86812 & -0.44137 \\
\hline $\mathrm{H}$ & 5.458053 & 1.914913 & -0.27191 \\
\hline $\mathrm{H}$ & 5.142369 & -2.27724 & 0.464203 \\
\hline $\mathrm{H}$ & 7.340347 & -2.06315 & -0.35308 \\
\hline $\mathrm{H}$ & 7.242535 & -1.74441 & 1.403602 \\
\hline $\mathrm{H}$ & 8.582627 & -1.05693 & 0.443416 \\
\hline $\mathrm{H}$ & 4.458619 & 3.825027 & 0.20738 \\
\hline $\mathrm{H}$ & 4.548384 & 3.477703 & -1.53314 \\
\hline $\mathrm{H}$ & 3.359581 & 4.631731 & -0.91766 \\
\hline $\mathrm{H}$ & 2.659212 & 2.265945 & -2.69856 \\
\hline $\mathrm{H}$ & 1.529832 & 3.476484 & -2.0642 \\
\hline $\mathrm{H}$ & 1.214362 & 1.748159 & -1.80339 \\
\hline $\mathrm{H}$ & 1.428149 & 3.97253 & 0.423759 \\
\hline $\mathrm{H}$ & 1.074589 & 2.268076 & 0.775391 \\
\hline $\mathrm{H}$ & 2.447379 & 3.076271 & 1.564767 \\
\hline $\mathrm{H}$ & 3.11904 & -3.34682 & 0.493881 \\
\hline $\mathrm{H}$ & -5.45803 & 1.914897 & 0.271775 \\
\hline
\end{tabular}




$\begin{array}{lrrr}\text { H } & -5.14233 & -2.27717 & -0.46473 \\ \text { H } & -7.34016 & -2.06288 & 0.353015 \\ \text { H } & -7.2427 & -1.7447 & -1.40378 \\ \text { H } & -8.58261 & -1.05694 & -0.44352 \\ \text { H } & -2.65941 & 2.265255 & 2.698724 \\ \text { H } & -1.21453 & 1.747701 & 1.803528 \\ \text { H } & -1.52999 & 3.475986 & 2.064836 \\ \text { H } & -1.07479 & 2.268142 & -0.77546 \\ \text { H } & -1.42771 & 3.97251 & -0.42277 \\ \text { H } & -2.44737 & 3.07734 & -1.56424 \\ \text { H } & -3.3597 & 4.631602 & 0.91811 \\ \text { H } & -4.45879 & 3.824927 & -0.20686 \\ \text { H } & -4.54836 & 3.477432 & 1.53363 \\ \text { H } & -3.11905 & -3.3469 & -0.49344 \\ \text { H } & 1.312673 & -4.67472 & 0.177115 \\ \text { H } & 0.329415 & -3.99052 & 1.493263 \\ \text { H } & -1.31279 & -4.67473 & -0.17577 \\ \text { H } & -0.3295 & -3.99132 & -1.4923\end{array}$

F) Optimized XYZ Coordinates $(\AA)$ for $\mathbf{6}$

$\begin{array}{lrrr}\text { Co } & -1.7 \mathrm{E}-05 & 0.816493 & -2.2 \mathrm{E}-06 \\ \mathrm{O} & -1.50785 & -0.28396 & -0.50396 \\ \mathrm{O} & 1.507829 & -0.28395 & 0.50396 \\ \mathrm{O} & -6.91964 & 0.014378 & 0.59905 \\ \mathrm{O} & 6.919627 & 0.014438 & -0.599 \\ \mathrm{~N} & -1.23961 & 2.228395 & 0.732776 \\ \mathrm{~N} & 1.239574 & 2.228396 & -0.73279 \\ \mathrm{C} & -2.78317 & -0.17172 & -0.22277 \\ \mathrm{C} & -3.69854 & -1.21712 & -0.62065 \\ \mathrm{C} & -5.04042 & -1.07197 & -0.30587 \\ \mathrm{C} & -5.56367 & 0.047592 & 0.37635 \\ \mathrm{C} & -4.71077 & 1.059967 & 0.754646 \\ \mathrm{C} & -3.32203 & 0.967114 & 0.462656 \\ \mathrm{C} & -7.49685 & 1.118385 & 1.280488 \\ \mathrm{C} & -3.19391 & -2.46657 & -1.37356 \\ \mathrm{C} & -2.53971 & -2.04903 & -2.71343 \\ \mathrm{C} & -2.16843 & -3.23231 & -0.50232 \\ \mathrm{C} & -4.33651 & -3.44764 & -1.70694 \\ \mathrm{C} & -2.52059 & 2.089806 & 0.877352 \\ \mathrm{C} & 2.783147 & -0.17172 & 0.222753 \\ \text { C } & 3.698521 & -1.21713 & 0.620618 \\ \text { C } & 5.040401 & -1.07195 & 0.305871 \\ \text { C } & 5.563656 & 0.047627 & -0.37633 \\ \text { C } & 4.710744 & 1.059992 & -0.75463 \\ \text { C } & 3.322001 & 0.967125 & -0.46266 \\ \text { C } & 7.496832 & 1.118463 & -1.28041 \\ \text { C } & 3.19395 & -2.46659 & 1.373537 \\ \text { C } & 4.336627 & -3.44758 & 1.706877\end{array}$




$\begin{array}{rrrr}\text { C } & 2.539777 & -2.04909 & 2.71343 \\ \mathrm{C} & 2.168503 & -3.2324 & 0.502315 \\ \mathrm{C} & 2.520558 & 2.089816 & -0.87735 \\ \mathrm{C} & -0.59029 & 3.464155 & 1.170326 \\ \mathrm{C} & -2.2 \mathrm{E}-05 & 4.285074 & -1.2 \mathrm{E}-05 \\ \mathrm{C} & 0.590248 & 3.464151 & -1.17034 \\ \mathrm{H} & -5.75684 & -1.83677 & -0.58222 \\ \mathrm{H} & -5.07114 & 1.940929 & 1.275378 \\ \mathrm{H} & -7.35495 & 2.054434 & 0.723931 \\ \mathrm{H} & -7.07601 & 1.234651 & 2.288146 \\ \mathrm{H} & -8.56414 & 0.903209 & 1.357957 \\ \mathrm{H} & -3.27022 & -1.54808 & -3.36071 \\ \mathrm{H} & -1.70079 & -1.37161 & -2.54723 \\ \mathrm{H} & -2.17387 & -2.93654 & -3.24521 \\ \mathrm{H} & -1.31131 & -2.60376 & -0.25596 \\ \mathrm{H} & -1.80858 & -4.11952 & -1.03872 \\ \mathrm{H} & -2.63204 & -3.5711 & 0.432402 \\ \mathrm{H} & -3.92384 & -4.31031 & -2.24222 \\ \mathrm{H} & -4.83264 & -3.8268 & -0.80592 \\ \mathrm{H} & -5.09711 & -2.99298 & -2.35239 \\ \mathrm{H} & -3.07394 & 2.906719 & 1.357684 \\ \mathrm{H} & 5.75683 & -1.83673 & 0.582241 \\ \mathrm{H} & 5.071111 & 1.940964 & -1.27535 \\ \mathrm{H} & 7.354898 & 2.054504 & -0.72385 \\ \mathrm{H} & 7.07602 & 1.234731 & -2.28808 \\ \mathrm{H} & 8.564136 & 0.903309 & -1.35785 \\ \mathrm{H} & 4.832675 & -3.82679 & 0.805834 \\ \mathrm{H} & 5.097274 & -2.99282 & 2.352201 \\ \mathrm{H} & 3.924061 & -4.31021 & 2.242274 \\ \mathrm{H} & 3.270274 & -1.54806 & 3.36067 \\ \mathrm{H} & 2.174047 & -2.93662 & 3.245242 \\ \mathrm{H} & 1.700784 & -1.37175 & 2.547266 \\ \mathrm{H} & -0.22198 & 3.179518 & -1.84854 \\ \mathrm{H} & 1.285461 & 4.095258 & -1.7406\end{array}$

G) Optimized XYZ Coordinates (Å) for $\mathbf{1}^{+}$

\begin{tabular}{|c|c|c|c|}
\hline V & 0.072361 & -0.89076 & -0.37673 \\
\hline 0 & 1.391443 & 0.142186 & 0.366811 \\
\hline 0 & -1.41847 & 0.126747 & -0.40113 \\
\hline 0 & 6.858302 & -0.19345 & -0.23184 \\
\hline
\end{tabular}




\begin{tabular}{|c|c|c|c|}
\hline 0 & -6.8633 & -0.16629 & 0.335118 \\
\hline 0 & 0.537937 & -0.97631 & -1.86652 \\
\hline 0 & -0.44214 & -0.96697 & 2.042842 \\
\hline$N$ & 1.247549 & -2.49241 & 0.229817 \\
\hline$N$ & -1.28361 & -2.50266 & -0.33971 \\
\hline C & 2.7253 & 0.067959 & 0.191762 \\
\hline C & 3.532094 & 1.239842 & 0.191804 \\
\hline C & 4.900373 & 1.047734 & 0.034875 \\
\hline C & 5.512163 & -0.21963 & -0.08663 \\
\hline C & 4.725882 & -1.35648 & -0.03644 \\
\hline C & 3.322889 & -1.21715 & 0.090554 \\
\hline C & 7.55043 & -1.43182 & -0.3602 \\
\hline C & 2.933988 & 2.653274 & 0.343485 \\
\hline C & 2.194866 & 2.766639 & 1.699222 \\
\hline C & 1.96268 & 2.942734 & -0.82612 \\
\hline C & 4.025113 & 3.743119 & 0.321458 \\
\hline C & 2.543028 & -2.41351 & 0.239632 \\
\hline C & -2.74484 & 0.058809 & -0.21633 \\
\hline C & -3.53809 & 1.239641 & -0.18269 \\
\hline C & -4.90391 & 1.060039 & 0.012918 \\
\hline C & -5.52129 & -0.20132 & 0.156832 \\
\hline C & -4.74228 & -1.34501 & 0.101842 \\
\hline C & -3.34523 & -1.21735 & -0.08219 \\
\hline C & -7.56443 & -1.4001 & 0.459495 \\
\hline$C$ & -2.92634 & 2.644025 & -0.35343 \\
\hline C & -3.99862 & 3.749589 & -0.27636 \\
\hline C & -2.24236 & 2.750003 & -1.73902 \\
\hline C & -1.90088 & 2.909806 & 0.775292 \\
\hline C & -2.5675 & -2.42145 & -0.1833 \\
\hline C & 0.572547 & -3.76463 & 0.493563 \\
\hline C & -0.6373 & -3.81448 & -0.43635 \\
\hline $\mathrm{H}$ & 5.570958 & 1.897073 & 0.003714 \\
\hline $\mathrm{H}$ & 5.153591 & -2.35035 & -0.10141 \\
\hline $\mathrm{H}$ & 7.424404 & -2.05188 & 0.53642 \\
\hline $\mathrm{H}$ & 7.210517 & -1.98638 & -1.24393 \\
\hline $\mathrm{H}$ & 8.602932 & -1.17275 & -0.47654 \\
\hline $\mathrm{H}$ & 2.879606 & 2.580979 & 2.534717 \\
\hline $\mathrm{H}$ & 1.361733 & 2.063156 & 1.771354 \\
\hline $\mathrm{H}$ & 1.789975 & 3.778051 & 1.816837 \\
\hline $\mathrm{H}$ & 1.126219 & 2.241587 & -0.8519 \\
\hline $\mathrm{H}$ & 1.552379 & 3.953882 & -0.72391 \\
\hline $\mathrm{H}$ & 2.484313 & 2.888651 & -1.78814 \\
\hline $\mathrm{H}$ & 3.55241 & 4.723395 & 0.439417 \\
\hline $\mathrm{H}$ & 4.57464 & 3.759767 & -0.62619 \\
\hline $\mathrm{H}$ & 4.745347 & 3.627198 & 1.139137 \\
\hline $\mathrm{H}$ & 3.105277 & -3.33781 & 0.39969 \\
\hline $\mathrm{H}$ & -5.56587 & 1.915795 & 0.055184 \\
\hline $\mathrm{H}$ & -5.17476 & -2.33525 & 0.189227 \\
\hline $\mathrm{H}$ & -7.44999 & -2.01402 & -0.44268 \\
\hline
\end{tabular}




$\begin{array}{lrrr}\text { H } & -7.22322 & -1.96457 & 1.336638 \\ \text { H } & -8.61379 & -1.13328 & 0.586085 \\ \text { H } & -4.5115 & 3.765127 & 0.692043 \\ \text { H } & -4.75109 & 3.651696 & -1.06658 \\ \text { H } & -3.5165 & 4.723811 & -0.40546 \\ \text { H } & -2.97017 & 2.596534 & -2.54352 \\ \text { H } & -1.81345 & 3.750861 & -1.86255 \\ \text { H } & -1.43927 & 2.020297 & -1.86218 \\ \text { H } & -1.47498 & 3.912707 & 0.660333 \\ \text { H } & -1.07561 & 2.194234 & 0.756027 \\ \text { H } & -2.38328 & 2.864858 & 1.759305 \\ \text { H } & -3.13164 & -3.35725 & -0.13216 \\ \text { H } & 1.245956 & -4.61389 & 0.331822 \\ \text { H } & 0.238638 & -3.77188 & 1.537409 \\ \text { H } & -1.32349 & -4.62327 & -0.1619 \\ \text { H } & -0.31148 & -3.96493 & -1.47246 \\ \text { H } & 0.256638 & -0.38291 & 2.382166 \\ \text { H } & -1.27733 & -0.54527 & 2.303732\end{array}$

H) Optimized XYZ Coordinates (Å) for $\mathbf{2}^{+}$

$\begin{array}{lrrr}\text { V } & 0.089672 & -0.84387 & 0.406252 \\ \text { O } & -1.36282 & 0.228471 & 0.606587 \\ \text { O } & 1.318919 & 0.268254 & -0.40642 \\ \text { O } & 6.793373 & -0.07005 & 0.147892 \\ \text { O } & -0.70052 & -0.81322 & -1.91095 \\ \text { H } & -1.56382 & -0.38693 & -2.04346 \\ \text { H } & -0.04668 & -0.20106 & -2.28944 \\ \text { N } & 1.19961 & -2.38088 & -0.47189 \\ \text { O } & -6.74329 & 0.040244 & -0.55513 \\ \text { N } & -1.31083 & -2.41564 & 0.664738 \\ \text { C } & -2.20299 & 2.930774 & 1.735387 \\ \text { H } & -1.75192 & 3.923605 & 1.843513 \\ \text { H } & -1.43369 & 2.184653 & 1.944111 \\ \text { H } & -2.98772 & 2.836222 & 2.494273 \\ \text { C } & -0.04853 & -4.4874 & 0.052364 \\ \text { H } & 0.724877 & -5.10393 & 0.523152 \\ \text { H } & -0.74224 & -5.17252 & -0.44794 \\ \text { C } & 4.82791 & 1.176058 & -0.02261 \\ \text { H } & 5.493671 & 2.02464 & 0.071 \\ \text { C } & 2.491709 & -2.26237 & -0.53897 \\ \text { H } & 3.056925 & -3.12623 & -0.9007 \\ \text { C } & -3.42962 & 1.384413 & 0.157697 \\ \text { C } & -3.30254 & -1.07827 & 0.192809 \\ \text { C } & 3.459127 & 1.371407 & -0.16628 \\ \text { C } & 3.264847 & -1.08638 & -0.24597 \\ \text { C } & -4.77989 & 1.231143 & -0.1396 \\ \text { H } & -5.41013 & 2.100739 & -0.27741 \\ \text { C } & 4.668381 & -1.22717 & -0.14127\end{array}$




\begin{tabular}{|c|c|c|c|}
\hline $\mathrm{H}$ & 5.102706 & -2.22004 & -0.16417 \\
\hline$C$ & 0.567133 & -3.58694 & -1.02641 \\
\hline $\mathrm{H}$ & 1.300349 & -4.15148 & -1.61325 \\
\hline $\mathrm{H}$ & -0.20624 & -3.23926 & -1.71612 \\
\hline$C$ & 2.65576 & 0.1979 & -0.24625 \\
\hline$C$ & -2.79789 & 2.781255 & 0.313248 \\
\hline$C$ & -2.66966 & 0.186768 & 0.301271 \\
\hline$C$ & 3.944933 & 3.880219 & -0.11875 \\
\hline $\mathrm{H}$ & 3.467939 & 4.864104 & -0.17324 \\
\hline $\mathrm{H}$ & 4.670308 & 3.823134 & -0.93797 \\
\hline $\mathrm{H}$ & 4.48855 & 3.83502 & 0.83139 \\
\hline$C$ & -7.47202 & -1.17941 & -0.66368 \\
\hline $\mathrm{H}$ & -7.45442 & -1.7376 & 0.280748 \\
\hline $\mathrm{H}$ & -7.07688 & -1.80769 & -1.47202 \\
\hline $\mathrm{H}$ & -8.49756 & -0.89197 & -0.89598 \\
\hline$C$ & -0.77929 & -3.69791 & 1.155932 \\
\hline $\mathrm{H}$ & -1.58906 & -4.29934 & 1.583661 \\
\hline $\mathrm{H}$ & -0.08853 & -3.45432 & 1.967249 \\
\hline$C$ & 5.44749 & -0.09304 & 0.001768 \\
\hline$C$ & 2.131476 & 2.989695 & -1.57375 \\
\hline $\mathrm{H}$ & 1.708492 & 3.999431 & -1.62338 \\
\hline $\mathrm{H}$ & 1.315274 & 2.27675 & -1.7092 \\
\hline $\mathrm{H}$ & 2.830127 & 2.878245 & -2.41089 \\
\hline$C$ & 2.858682 & 2.79005 & -0.22169 \\
\hline$C$ & 1.882105 & 2.994313 & 0.960985 \\
\hline $\mathrm{H}$ & 2.404688 & 2.887572 & 1.918197 \\
\hline $\mathrm{H}$ & 1.056217 & 2.281275 & 0.942647 \\
\hline $\mathrm{H}$ & 1.4587 & 4.004494 & 0.92178 \\
\hline$C$ & -2.59033 & -2.28915 & 0.512075 \\
\hline $\mathrm{H}$ & -3.21309 & -3.17278 & 0.6827 \\
\hline$C$ & -3.83499 & 3.905922 & 0.121157 \\
\hline $\mathrm{H}$ & -4.64217 & 3.852034 & 0.859723 \\
\hline $\mathrm{H}$ & -4.27833 & 3.896921 & -0.88102 \\
\hline $\mathrm{H}$ & -3.3394 & 4.873413 & 0.249708 \\
\hline$C$ & -1.69769 & 2.972111 & -0.75788 \\
\hline $\mathrm{H}$ & -2.1217 & 2.904534 & -1.76761 \\
\hline $\mathrm{H}$ & -0.8997 & 2.233212 & -0.66046 \\
\hline $\mathrm{H}$ & -1.24561 & 3.964725 & -0.65545 \\
\hline$C$ & -4.68637 & -1.17782 & -0.07845 \\
\hline $\mathrm{H}$ & -5.14385 & -2.1592 & -0.13154 \\
\hline$C$ & 7.494275 & -1.30989 & 0.17202 \\
\hline $\mathrm{H}$ & 7.158188 & -1.93942 & 1.00553 \\
\hline $\mathrm{H}$ & 8.544908 & -1.05405 & 0.310472 \\
\hline $\mathrm{H}$ & 7.372744 & -1.85304 & -0.77374 \\
\hline$C$ & -5.42223 & -0.01921 & -0.26599 \\
\hline 0 & 0.746247 & -0.96266 & 1.818804 \\
\hline
\end{tabular}

I) Optimized XYZ Coordinates (Å) for $\mathbf{3}^{+}$ 


\begin{tabular}{|c|c|c|c|}
\hline $\mathrm{Cu}$ & -0.04817 & 1.113781 & -0.0403 \\
\hline 0 & -1.35656 & -0.16878 & -0.14438 \\
\hline 0 & 1.42355 & -0.16465 & 0.088735 \\
\hline 0 & -6.80724 & 0.15085 & 0.202435 \\
\hline 0 & 6.781636 & 0.191641 & -0.10014 \\
\hline$N$ & -1.26721 & 2.549896 & 0.274689 \\
\hline$N$ & 1.277656 & 2.55422 & -0.31393 \\
\hline C & -2.64717 & -0.0431 & -0.05664 \\
\hline C & -3.47617 & -1.20363 & -0.20361 \\
\hline C & -4.83188 & -1.03751 & -0.10184 \\
\hline C & -5.46029 & 0.201898 & 0.134963 \\
\hline C & -4.69128 & 1.315138 & 0.270686 \\
\hline C & -3.27888 & 1.208986 & 0.177915 \\
\hline C & -7.50518 & 1.345982 & 0.434903 \\
\hline C & -2.86483 & -2.5839 & -0.46857 \\
\hline C & -3.93828 & -3.66961 & -0.58807 \\
\hline C & -2.08296 & -2.56678 & -1.7912 \\
\hline C & -1.93956 & -2.98083 & 0.691805 \\
\hline C & -2.55214 & 2.417567 & 0.3169 \\
\hline$C$ & 2.668486 & -0.05157 & 0.048413 \\
\hline C & 3.511895 & -1.23569 & 0.213247 \\
\hline C & 4.860208 & -1.06098 & 0.147441 \\
\hline$C$ & 5.466023 & 0.200837 & -0.06271 \\
\hline$C$ & 4.67459 & 1.34552 & -0.20991 \\
\hline$C$ & 3.304443 & 1.240724 & -0.15924 \\
\hline$C$ & 7.517616 & 1.389353 & -0.30485 \\
\hline$C$ & 2.882973 & -2.60673 & 0.44899 \\
\hline$C$ & 2.066765 & -2.58624 & 1.753206 \\
\hline$C$ & 1.981614 & -2.9829 & -0.73997 \\
\hline C & 3.954349 & -3.69206 & 0.585563 \\
\hline$C$ & 2.54521 & 2.468545 & -0.31241 \\
\hline$C$ & -0.63015 & 3.843461 & 0.414673 \\
\hline$C$ & 0.607637 & 3.838997 & -0.48088 \\
\hline $\mathrm{H}$ & -5.49416 & -1.87752 & -0.20329 \\
\hline $\mathrm{H}$ & -5.12563 & 2.283326 & 0.450012 \\
\hline $\mathrm{H}$ & -7.32766 & 2.069073 & -0.36005 \\
\hline $\mathrm{H}$ & -7.2275 & 1.786826 & 1.391369 \\
\hline $\mathrm{H}$ & -8.55535 & 1.085928 & 0.452967 \\
\hline $\mathrm{H}$ & -4.52124 & -3.77274 & 0.324065 \\
\hline $\mathrm{H}$ & -4.62089 & -3.48343 & -1.41378 \\
\hline $\mathrm{H}$ & -3.45648 & -4.62563 & -0.77507 \\
\hline $\mathrm{H}$ & -2.74194 & -2.32802 & -2.62332 \\
\hline $\mathrm{H}$ & -1.65483 & -3.54945 & -1.97979 \\
\hline $\mathrm{H}$ & -1.27952 & -1.84099 & -1.77122 \\
\hline $\mathrm{H}$ & -1.51527 & -3.96555 & 0.504036 \\
\hline $\mathrm{H}$ & -1.12846 & -2.27426 & 0.813353 \\
\hline $\mathrm{H}$ & -2.49576 & -3.03286 & 1.625466 \\
\hline $\mathrm{H}$ & -3.1461 & 3.313982 & 0.469105 \\
\hline $\mathrm{H}$ & 5.536128 & -1.88848 & 0.255349 \\
\hline
\end{tabular}




$\begin{array}{lrrr}\text { H } & 5.130157 & 2.30817 & -0.36344 \\ \text { H } & 7.328584 & 2.096035 & 0.497528 \\ \text { H } & 7.271735 & 1.829443 & -1.2665 \\ \text { H } & 8.55704 & 1.095488 & -0.29383 \\ \text { H } & 2.696695 & -2.33118 & 2.602762 \\ \text { H } & 1.241168 & -1.88744 & 1.707254 \\ \text { H } & 1.656554 & -3.57656 & 1.932151 \\ \text { H } & 1.144254 & -2.30598 & -0.84919 \\ \text { H } & 1.585701 & -3.9827 & -0.58216 \\ \text { H } & 2.548185 & -2.99576 & -1.66868 \\ \text { H } & 3.465964 & -4.64699 & 0.753323 \\ \text { H } & 4.556838 & -3.79148 & -0.31439 \\ \text { H } & 4.61566 & -3.51522 & 1.430676 \\ \text { H } & 3.139127 & 3.369849 & -0.43534 \\ \text { H } & -1.29135 & 4.661112 & 0.13578 \\ \text { H } & -0.32692 & 3.995042 & 1.449977 \\ \text { H } & 1.271902 & 4.670166 & -0.25648 \\ \text { H } & 0.297804 & 3.913831 & -1.52133\end{array}$

J) Optimized XYZ Coordinates (Å) for $\mathbf{4}^{+}$

$\begin{array}{lrrr}\mathrm{Cu} & 0.049561 & 1.006804 & 0.046435 \\ \mathrm{O} & 1.349019 & -0.27076 & 0.249219 \\ \mathrm{O} & -1.41297 & -0.29086 & -0.13813 \\ \mathrm{O} & 6.784998 & -0.02291 & -0.32776 \\ \mathrm{O} & -6.76783 & 0.014516 & 0.142294 \\ \mathrm{~N} & 1.234428 & 2.35678 & -0.63155 \\ \mathrm{~N} & -1.26822 & 2.353223 & 0.668465 \\ \mathrm{C} & 2.638134 & -0.16794 & 0.100983 \\ \mathrm{C} & 3.475021 & -1.28725 & 0.413466 \\ \mathrm{C} & 4.82723 & -1.14088 & 0.244228 \\ \mathrm{C} & 5.43938 & 0.036345 & -0.22797 \\ \mathrm{C} & 4.657872 & 1.10514 & -0.54072 \\ \mathrm{C} & 3.251332 & 1.018718 & -0.37534 \\ \mathrm{C} & 7.46444 & 1.109929 & -0.8002 \\ \mathrm{C} & 2.87685 & -2.60681 & 0.912757 \\ \mathrm{C} & 2.106713 & -2.38127 & 2.223018 \\ \mathrm{C} & 1.943419 & -3.189 & -0.15964 \\ \mathrm{C} & 3.958283 & -3.6534 & 1.195747 \\ \mathrm{C} & 2.509816 & 2.174718 & -0.73509 \\ \mathrm{C} & -2.65726 & -0.19501 & -0.06994 \\ \mathrm{C} & -3.50029 & -1.33627 & -0.4267 \\ \mathrm{C} & -4.8485 & -1.17483 & -0.33089 \\ \mathrm{C} & -5.45231 & 0.028963 & 0.104504 \\ \mathrm{C} & -4.65903 & 1.126015 & 0.461641 \\ \mathrm{C} & -3.29037 & 1.036017 & 0.374238 \\ \mathrm{C} & -7.5019 & 1.15357 & 0.568642 \\ \mathrm{C} & -2.87041 & -2.65319 & -0.87231 \\ \mathrm{C} & -3.94123 & -3.69362 & -1.21134 \\ & & & \\ & & & \\ & & & \end{array}$




\begin{tabular}{rrrr} 
C & -2.01525 & -2.43352 & -2.13243 \\
C & -2.00641 & -3.21943 & 0.268255 \\
C & -2.5272 & 2.207735 & 0.76801 \\
C & 0.639966 & 3.598844 & -1.09179 \\
C & -0.00722 & 4.411286 & 0.036125 \\
C & -0.64246 & 3.579181 & 1.15425 \\
H & 5.496625 & -1.95061 & 0.46982 \\
H & 5.079417 & 2.023609 & -0.91085 \\
H & 7.305658 & 1.967974 & -0.14817 \\
H & 7.153461 & 1.365123 & -1.81244 \\
H & 8.515939 & 0.854376 & -0.80371 \\
H & 2.768751 & -1.99577 & 2.995696 \\
H & 1.289444 & -1.68324 & 2.091439 \\
H & 1.698985 & -3.32578 & 2.578099 \\
H & 1.138599 & -2.50521 & -0.39893 \\
H & 1.510729 & -4.12307 & 0.194434 \\
H & 2.495946 & -3.40424 & -1.07187 \\
H & 3.484995 & -4.56738 & 1.544773 \\
H & 4.530486 & -3.90396 & 0.305736 \\
H & 4.650103 & -3.32951 & 1.969758 \\
H & 3.093346 & 2.992738 & -1.14766 \\
H & -5.52523 & -1.97046 & -0.58066 \\
H & -5.11323 & 2.04058 & 0.800914 \\
H & -7.31151 & 1.998356 & -0.08637 \\
H & -7.25568 & 1.404447 & 1.595934 \\
H & -8.54178 & 0.868663 & 0.5026 \\
H & -4.56602 & -3.93629 & -0.35498 \\
H & -4.58102 & -3.3743 & -2.03094 \\
H & -3.45191 & -4.61144 & -1.52241 \\
H & -2.61361 & -2.02275 & -2.94303 \\
H & -1.62231 & -3.39033 & -2.46572 \\
H & -1.17633 & -1.77488 & -1.94752 \\
H & -1.18617 & -2.56141 & 0.525588 \\
H & -2.60685 & -3.39746 & 1.157811 \\
H & -1.5864 & -4.17239 & -0.04283 \\
H & -3.11616 & 3.017522 & 1.189588 \\
H & 1.37877 & 4.21467 & -1.60051 \\
\hline & -0.11871 & 3.348954 & -1.8304 \\
\hline & -1.732421 & 5.055507 & 0.504614 \\
H & -1275532 & 5.063974 & -0.40747 \\
\hline & 3.27065 & 1.855473 \\
H & 4.181982 & 1.705093
\end{tabular}

K) Optimized XYZ Coordinates (Å) for $\mathbf{5}^{+}$

\begin{tabular}{|c|c|c|c|}
\hline Co & -0.01283 & -1.16565 & 0.083461 \\
\hline 0 & 0.211414 & -1.2063 & 2.240829 \\
\hline 0 & -1.30825 & 0.143996 & -0.07 \\
\hline
\end{tabular}




\begin{tabular}{|c|c|c|c|}
\hline 0 & 1.297147 & 0.151922 & 0.067085 \\
\hline 0 & -6.81404 & -0.01048 & -0.05244 \\
\hline 0 & 6.781496 & -0.07463 & -0.37695 \\
\hline $\mathrm{N}$ & -1.29283 & -2.53686 & 0.138557 \\
\hline $\mathrm{N}$ & 1.216214 & -2.51648 & -0.33862 \\
\hline C & -2.62028 & 0.066429 & -0.09199 \\
\hline C & -3.40441 & 1.264235 & -0.27691 \\
\hline C & -4.78144 & 1.135497 & -0.24235 \\
\hline $\mathrm{H}$ & -5.41564 & 2.005466 & -0.3596 \\
\hline C & -5.45622 & -0.09672 & -0.06309 \\
\hline C & -4.72135 & -1.25567 & 0.070663 \\
\hline $\mathrm{H}$ & -5.19301 & -2.22507 & 0.184549 \\
\hline C & -3.30565 & -1.18165 & 0.055352 \\
\hline C & -2.74288 & 2.636196 & -0.50974 \\
\hline C & -3.78525 & 3.759895 & -0.68174 \\
\hline $\mathrm{H}$ & -4.44267 & 3.587797 & -1.54148 \\
\hline $\mathrm{H}$ & -3.26144 & 4.706356 & -0.85343 \\
\hline $\mathrm{H}$ & -4.4085 & 3.886654 & 0.210908 \\
\hline C & -1.85428 & 3.010772 & 0.700418 \\
\hline $\mathrm{H}$ & -2.45323 & 3.062529 & 1.617851 \\
\hline $\mathrm{H}$ & -1.40387 & 3.997268 & 0.538359 \\
\hline $\mathrm{H}$ & -1.05175 & 2.287453 & 0.846533 \\
\hline C & -1.89839 & 2.5853 & -1.80667 \\
\hline $\mathrm{H}$ & -1.12517 & 1.817089 & -1.75376 \\
\hline $\mathrm{H}$ & -1.41158 & 3.553627 & -1.97239 \\
\hline $\mathrm{H}$ & -2.53685 & 2.377448 & -2.67367 \\
\hline C & -7.55819 & -1.2138 & 0.124534 \\
\hline $\mathrm{H}$ & -7.36662 & -1.92254 & -0.69023 \\
\hline $\mathrm{H}$ & -8.60833 & -0.92018 & 0.111908 \\
\hline $\mathrm{H}$ & -7.3218 & -1.68828 & 1.084742 \\
\hline C & -2.58749 & -2.41087 & 0.140479 \\
\hline $\mathrm{H}$ & -3.18094 & -3.32481 & 0.200144 \\
\hline C & -0.67866 & -3.87127 & 0.236997 \\
\hline $\mathrm{H}$ & -0.42055 & -4.05918 & 1.285776 \\
\hline $\mathrm{H}$ & -1.36829 & -4.65068 & -0.09985 \\
\hline C & 0.583849 & -3.82068 & -0.61169 \\
\hline $\mathrm{H}$ & 0.324822 & -3.86904 & -1.67585 \\
\hline $\mathrm{H}$ & 1.265857 & -4.64416 & -0.38017 \\
\hline C & 2.506859 & -2.39001 & -0.47365 \\
\hline $\mathrm{H}$ & 3.071543 & -3.28524 & -0.74087 \\
\hline C & 3.252482 & -1.18968 & -0.30637 \\
\hline C & 4.662907 & -1.27894 & -0.43839 \\
\hline $\mathrm{H}$ & 5.106438 & -2.2436 & -0.65603 \\
\hline C & 5.425847 & -0.14337 & -0.28197 \\
\hline C & 4.78689 & 1.087774 & 0.010892 \\
\hline $\mathrm{H}$ & 5.444449 & 1.939965 & 0.129416 \\
\hline C & 3.419355 & 1.236517 & 0.145442 \\
\hline C & 2.602131 & 0.056813 & -0.02639 \\
\hline C & 2.802029 & 2.611441 & 0.46869 \\
\hline
\end{tabular}




$\begin{array}{lrrr}\text { C } & 1.865353 & 3.047587 & -0.68275 \\ \text { H } & 2.425508 & 3.139974 & -1.62094 \\ \text { H } & 1.427396 & 4.026894 & -0.4554 \\ \text { H } & 1.053663 & 2.33469 & -0.83006 \\ \text { C } & 2.018598 & 2.53908 & 1.802283 \\ \text { H } & 1.188333 & 1.833096 & 1.741323 \\ \text { H } & 1.606105 & 3.525106 & 2.046022 \\ \text { H } & 2.678452 & 2.238095 & 2.624744 \\ \text { C } & 3.878255 & 3.704606 & 0.630849 \\ \text { H } & 4.571617 & 3.485985 & 1.451062 \\ \text { H } & 3.386188 & 4.655702 & 0.860699 \\ \text { H } & 4.46093 & 3.850245 & -0.2857 \\ \text { C } & 7.490963 & -1.27568 & -0.67191 \\ \text { H } & 7.190773 & -1.68468 & -1.6443 \\ \text { H } & 7.332303 & -2.03149 & 0.106929 \\ \text { H } & 8.545574 & -0.99996 & -0.7025 \\ \text { H } & -0.60782 & -1.2633 & 2.761856 \\ \text { H } & 0.632537 & -0.37224 & 2.51187\end{array}$

L) Optimized XYZ Coordinates ( $(\AA)$ for $\boldsymbol{6}^{+}$

$\begin{array}{lrrr}\text { Co } & -0.00657 & -0.81241 & 0.446083 \\ \text { O } & 1.470077 & 0.357395 & -0.01357 \\ \text { O } & -1.47015 & 0.383988 & 0.293242 \\ \text { O } & 6.91488 & 0.067683 & -0.3088 \\ \text { O } & -6.79396 & -0.32996 & -0.90615 \\ \text { N } & 1.42847 & -2.26933 & 0.793656 \\ \text { N } & -1.00253 & -2.20783 & -0.66758 \\ \text { C } & 2.754903 & 0.258409 & -0.0817 \\ \text { C } & 3.547262 & 1.401456 & -0.5048 \\ \text { C } & 4.916198 & 1.244615 & -0.54963 \\ \text { C } & 5.571679 & 0.03486 & -0.20323 \\ \text { C } & 4.825367 & -1.06749 & 0.20639 \\ \text { C } & 3.428904 & -0.97327 & 0.267045 \\ \text { C } & 7.665567 & -1.10905 & 0.010284 \\ \text { C } & 2.876696 & 2.733111 & -0.88992 \\ \text { C } & 1.922441 & 2.516429 & -2.09114 \\ \text { C } & 2.089648 & 3.298315 & 0.319189 \\ \text { C } & 3.914382 & 3.795406 & -1.30602 \\ \text { C } & 2.710109 & -2.15902 & 0.683852 \\ \text { C } & -2.73339 & 0.182477 & 0.011387 \\ \text { C } & -3.70963 & 1.210153 & 0.282084 \\ \text { C } & -5.02873 & 0.949367 & -0.0484 \\ \text { C } & -5.457 & -0.25572 & -0.64962 \\ \text { C } & -4.52882 & -1.2408 & -0.93144 \\ \text { C } & -3.16849 & -1.04086 & -0.59664 \\ \text { C } & -7.29298 & -1.51535 & -1.51935 \\ \text { C } & -3.29904 & 2.556557 & 0.91335 \\ \text { C } & -4.51368 & 3.478204 & 1.146977\end{array}$




\begin{tabular}{|c|c|c|c|}
\hline C & -2.62625 & 2.328386 & 2.289627 \\
\hline C & -2.32184 & 3.299323 & -0.03063 \\
\hline C & -2.26636 & -2.11155 & -0.94634 \\
\hline C & 0.861154 & -3.56936 & 1.163212 \\
\hline C & 0.248493 & -4.29504 & -0.06003 \\
\hline C & -0.26354 & -3.36901 & -1.17631 \\
\hline $\mathrm{H}$ & 5.560571 & 2.05813 & -0.85851 \\
\hline $\mathrm{H}$ & 5.304246 & -2.00144 & 0.477406 \\
\hline $\mathrm{H}$ & 7.390419 & -1.93865 & -0.64985 \\
\hline $\mathrm{H}$ & 7.513791 & -1.39754 & 1.055901 \\
\hline $\mathrm{H}$ & 8.709943 & -0.84239 & -0.14966 \\
\hline $\mathrm{H}$ & 2.472602 & 2.138798 & -2.96111 \\
\hline $\mathrm{H}$ & 1.124391 & 1.811748 & -1.85133 \\
\hline $\mathrm{H}$ & 1.464993 & 3.47211 & -2.37264 \\
\hline $\mathrm{H}$ & 1.29048 & 2.623945 & 0.630862 \\
\hline $\mathrm{H}$ & 1.641174 & 4.260811 & 0.046567 \\
\hline $\mathrm{H}$ & 2.757185 & 3.469027 & 1.17221 \\
\hline $\mathrm{H}$ & 3.390449 & 4.720038 & -1.56989 \\
\hline $\mathrm{H}$ & 4.611411 & 4.032806 & -0.4943 \\
\hline $\mathrm{H}$ & 4.494924 & 3.485408 & -2.18229 \\
\hline $\mathrm{H}$ & 3.336425 & -3.02819 & 0.913667 \\
\hline $\mathrm{H}$ & -5.80157 & 1.683749 & 0.142664 \\
\hline $\mathrm{H}$ & -4.8184 & -2.17304 & -1.40327 \\
\hline $\mathrm{H}$ & -7.11105 & -2.39575 & -0.89073 \\
\hline $\mathrm{H}$ & -6.84177 & -1.67317 & -2.50667 \\
\hline $\mathrm{H}$ & -8.36743 & -1.36471 & -1.63096 \\
\hline $\mathrm{H}$ & -5.02782 & 3.728293 & 0.212286 \\
\hline $\mathrm{H}$ & -5.24311 & 3.034155 & 1.834154 \\
\hline $\mathrm{H}$ & -4.16942 & 4.417531 & 1.592984 \\
\hline $\mathrm{H}$ & -3.28647 & 1.767127 & 2.96177 \\
\hline $\mathrm{H}$ & -2.4072 & 3.292975 & 2.762553 \\
\hline $\mathrm{H}$ & -1.68427 & 1.78662 & 2.182431 \\
\hline $\mathrm{H}$ & -1.42245 & 2.709874 & -0.21836 \\
\hline $\mathrm{H}$ & -2.80271 & 3.516247 & -0.99175 \\
\hline $\mathrm{H}$ & -2.02329 & 4.254426 & 0.418733 \\
\hline $\mathrm{H}$ & -2.72261 & -2.92594 & -1.5195 \\
\hline $\mathrm{H}$ & 1.617187 & -4.20816 & 1.63512 \\
\hline $\mathrm{H}$ & 0.080307 & -3.37516 & 1.903333 \\
\hline $\mathrm{H}$ & 0.998825 & -4.95496 & -0.50933 \\
\hline $\mathrm{H}$ & -0.567 & -4.93191 & 0.299222 \\
\hline $\mathrm{H}$ & 0.588538 & -2.98256 & -1.74599 \\
\hline $\mathrm{H}$ & -0.88407 & -3.94839 & -1.87092 \\
\hline 0 & -0.37862 & -0.88853 & 2.553563 \\
\hline $\mathrm{H}$ & -1.00689 & -0.19897 & 2.827656 \\
\hline $\mathrm{H}$ & 0.359195 & 7 & 3.1853 \\
\hline
\end{tabular}

M) Optimized XYZ Coordinates $(\AA \AA)$ for high-spin $\left(5^{+}\right)_{2}\left(\mu-\mathrm{OAc}^{-}\right)(S=2)$

Co $\quad \begin{array}{llll}\text { C } & -1.61352575 & 2.52702450 & -1.02349003\end{array}$ 


\begin{tabular}{|c|c|c|c|}
\hline Co & 1.68496299 & -2.62887488 & -1.07896394 \\
\hline 0 & -1.11528674 & 2.89249201 & 0.73122579 \\
\hline 0 & 3.04895565 & -1.55418640 & -0.43130153 \\
\hline $\mathrm{O}$ & -3.23964529 & 1.90309097 & -0.38242795 \\
\hline $\mathrm{O}$ & 2.85948080 & 6.02426160 & 2.92689304 \\
\hline $\mathrm{O}$ & -2.31268585 & -6.62322425 & 2.91108390 \\
\hline 0 & 1.36732458 & -3.19284091 & 0.65641905 \\
\hline $\mathrm{C}$ & -4.24722744 & 1.21385684 & -2.45077957 \\
\hline C & -0.16550073 & 3.65841308 & 1.22076713 \\
\hline 0 & 0.49536986 & -1.08761963 & -1.64974291 \\
\hline $\mathrm{C}$ & 1.83301073 & 5.11953554 & 0.90867854 \\
\hline $\mathrm{H}$ & 2.53020104 & 5.59041399 & 0.22455478 \\
\hline $\mathrm{C}$ & 1.92333448 & 5.28766477 & 2.27076763 \\
\hline $\mathrm{C}$ & 0.79189186 & 4.31005638 & 0.37767642 \\
\hline $\mathrm{C}$ & -5.26661281 & 0.50223390 & -3.13702855 \\
\hline $\mathrm{H}$ & -5.23659582 & 0.45887889 & -4.22015525 \\
\hline $\mathrm{N}$ & -2.30743620 & 2.62928753 & -2.78518640 \\
\hline $\mathrm{O}$ & -0.61181174 & 0.81492519 & -1.59883134 \\
\hline $\mathrm{C}$ & -2.49426379 & 3.71055256 & 3.31094152 \\
\hline $\mathrm{H}$ & -2.56777112 & 4.79995260 & 3.41198650 \\
\hline $\mathrm{H}$ & -3.20692993 & 3.26153604 & 4.01274676 \\
\hline $\mathrm{H}$ & -2.78933882 & 3.43472059 & 2.29869090 \\
\hline $\mathrm{C}$ & -0.29158712 & -0.28410818 & -1.05583781 \\
\hline $\mathrm{C}$ & -0.39735231 & -4.60838264 & -1.08674336 \\
\hline $\mathrm{H}$ & -1.09033483 & -5.23081382 & -1.65902335 \\
\hline C & 0.49491822 & -4.04537877 & 1.15699525 \\
\hline $\mathrm{N}$ & 2.51805338 & -2.80239988 & -2.76894040 \\
\hline C & -0.06528607 & 3.86385341 & 2.64883229 \\
\hline $\mathrm{C}$ & 0.96909185 & 4.65802345 & 3.10760698 \\
\hline $\mathrm{H}$ & 1.09178396 & 4.83697104 & 4.16837317 \\
\hline $\mathrm{N}$ & 0.42191140 & -3.85678548 & -1.75948042 \\
\hline $\mathrm{N}$ & -0.17908743 & 3.53164439 & -1.70700393 \\
\hline$C$ & -4.22323020 & 1.30762717 & -1.02198423 \\
\hline $\mathrm{C}$ & 0.72233961 & 4.18872984 & -1.03523959 \\
\hline $\mathrm{H}$ & 1.49702876 & 4.70134805 & -1.61106539 \\
\hline $\mathrm{C}$ & 5.51184122 & 1.48567862 & 1.63268339 \\
\hline $\mathrm{H}$ & 6.56449457 & 1.19299414 & 1.54876084 \\
\hline $\mathrm{H}$ & 5.30758150 & 1.66655193 & 2.69325998 \\
\hline $\mathrm{H}$ & 5.37337496 & 2.43717328 & 1.10568649 \\
\hline $\mathrm{C}$ & 3.53594312 & -2.10855253 & -3.19935758 \\
\hline $\mathrm{H}$ & 3.86872908 & -2.29376154 & -4.22425234 \\
\hline $\mathrm{C}$ & -0.47546834 & -5.18155442 & 3.05293861 \\
\hline $\mathrm{H}$ & -0.53074265 & -5.40875358 & 4.11022866 \\
\hline$C$ & 3.97875879 & -0.89666319 & -1.07751140 \\
\hline$C$ & 4.78020802 & 0.08182915 & -0.37518646 \\
\hline $\mathrm{C}$ & -0.76408469 & 3.62414575 & 5.09875552 \\
\hline $\mathrm{H}$ & -0.82977917 & 4.70646311 & 5.25764341 \\
\hline $\mathrm{H}$ & 0.22356645 & 3.28373974 & 5.43032623 \\
\hline $\mathrm{H}$ & -1.50645405 & 3.15300132 & 5.75161667 \\
\hline$C$ & 4.26047267 & -1.13672224 & -2.46288076 \\
\hline$C$ & -3.32215464 & 1.95477319 & -3.23807241 \\
\hline $\mathrm{H}$ & -3.51439114 & 1.99378428 & -4.31374007 \\
\hline
\end{tabular}




\begin{tabular}{|c|c|c|c|}
\hline E & -1.41986614 & -5.61076943 & 0.87944262 \\
\hline $\mathrm{H}$ & -2.11709728 & -6.10425944 & 0.21136736 \\
\hline F & 5.29741193 & -0.44027364 & -3.14501057 \\
\hline $\mathrm{H}$ & 5.47519363 & -0.66274881 & -4.19133774 \\
\hline 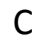 & -1.44142950 & -5.82201799 & 2.24017516 \\
\hline U & 7.05471760 & 1.24274905 & -2.97859493 \\
\hline C & -3.29426092 & -7.32031738 & 2.15720737 \\
\hline $\mathrm{H}$ & -3.87801027 & -7.89146923 & 2.88017018 \\
\hline t & -2.83141884 & -8.00892071 & 1.43796632 \\
\hline $\mathrm{H}$ & -3.95547696 & -6.62563000 & 1.62227621 \\
\hline 0 & -7.29226114 & -0.83879420 & -2.91862871 \\
\hline C & -0.45284624 & -4.72578716 & 0.33219778 \\
\hline C & 0.41711561 & -3.85398947 & -3.22540055 \\
\hline$\dashv$ & -0.10233825 & -2.95122581 & -3.56577081 \\
\hline $\mathrm{H}$ & -0.09208708 & -4.73719808 & -3.62695371 \\
\hline C & 1.34792961 & -4.16140419 & 4.97706707 \\
\hline$\dashv$ & 2.12357617 & -3.70491349 & 5.60089593 \\
\hline H & 1.43683294 & -5.24783791 & 5.08943338 \\
\hline H & 0.37803712 & -3.84908894 & 5.38112502 \\
\hline C & 3.11131115 & 0.89488568 & 1.34548251 \\
\hline $\mathrm{H}$ & 2.95311711 & 1.10367879 & 2.41010447 \\
\hline $\mathrm{H}$ & 2.37429897 & 0.15701553 & 1.02643314 \\
\hline $\mathrm{H}$ & 2.93322516 & 1.82578218 & 0.79381856 \\
\hline C & 1.54660417 & -3.70586956 & 3.51695357 \\
\hline C & -6.26787612 & -0.10134256 & -2.41066680 \\
\hline C & -6.29349936 & 0.06281240 & -1.00406449 \\
\hline H & -7.13622863 & -0.39102488 & -0.49799031 \\
\hline C & 3.84420966 & 6.69398875 & 2.15220359 \\
\hline $\mathrm{H}$ & 4.48302159 & 7.21632998 & 2.86541425 \\
\hline$H$ & 3.38785270 & 7.42302767 & 1.46977128 \\
\hline$H$ & 4.44874576 & 5.98266736 & 1.57383499 \\
\hline C & 1.88278365 & -3.79767126 & -3.64556659 \\
\hline $\mathrm{H}$ & 1.99424000 & -3.52429260 & -4.70089379 \\
\hline $\mathrm{H}$ & 2.35644301 & -4.77525611 & -3.48802935 \\
\hline C & 5.76362931 & 0.73325040 & -1.09382275 \\
\hline $\mathrm{H}$ & 6.38816864 & 1.47838972 & -0.61675961 \\
\hline C & 1.44371485 & -2.16217258 & 3.49571751 \\
\hline $\mathrm{H}$ & 0.44719787 & -1.83541987 & 3.81694197 \\
\hline $\mathrm{H}$ & 1.64051935 & -1.76157558 & 2.50092153 \\
\hline $\mathrm{H}$ & 2.17586186 & -1.73286643 & 4.18986500 \\
\hline C & -0.97087094 & 1.68717193 & 3.54890278 \\
\hline $\mathrm{H}$ & -1.68833246 & 1.22988440 & 4.24049905 \\
\hline $\mathrm{H}$ & 0.03186106 & 1.34229393 & 3.82897992 \\
\hline $\mathrm{H}$ & -1.18945048 & 1.33093109 & 2.54227572 \\
\hline C & 4.55660045 & 0.38956272 & 1.11801387 \\
\hline C & -5.51328857 & 0.99033361 & 1.22933006 \\
\hline C & -1.06124993 & 3.22885757 & 3.63770281 \\
\hline C & 4.82074632 & -0.88637822 & 1.95245195 \\
\hline $\mathrm{H}$ & 5.85634646 & -1.22335544 & 1.82557737 \\
\hline $\mathrm{H}$ & 4.15376432 & -1.69910798 & 1.66437497 \\
\hline$H$ & 4.66582255 & -0.67376323 & 3.01698204 \\
\hline C & -0.08017184 & 3.42005546 & -3.16628088 \\
\hline
\end{tabular}




\begin{tabular}{|c|c|c|c|}
\hline $\mathrm{H}$ & 0.53731822 & 4.22109717 & -3.58787053 \\
\hline $\mathrm{H}$ & 0.37289492 & 2.45020031 & -3.39824861 \\
\hline C & -5.34338410 & 0.76636408 & -0.28617876 \\
\hline C & 7.42105220 & 1.02406225 & -4.33338844 \\
\hline $\mathrm{H}$ & 8.25094664 & 1.70295565 & -4.53391565 \\
\hline $\mathrm{H}$ & 6.59060153 & 1.25277461 & -5.01485552 \\
\hline $\mathrm{H}$ & 7.74922570 & -0.01091766 & -4.49609741 \\
\hline C & 0.49823673 & -4.32356593 & 2.57125193 \\
\hline C & -1.50986604 & 3.46396626 & -3.69267696 \\
\hline $\mathrm{H}$ & -1.89320619 & 4.49185281 & -3.65791798 \\
\hline $\mathrm{H}$ & -1.57779782 & 3.10452945 & -4.72571988 \\
\hline C & 2.96018559 & -4.15976286 & 3.07674629 \\
\hline $\mathrm{H}$ & 3.71602960 & -3.71875046 & 3.73704168 \\
\hline $\mathrm{H}$ & 3.18094790 & -3.85493815 & 2.05238425 \\
\hline $\mathrm{H}$ & 3.05329161 & -5.25019644 & 3.14313947 \\
\hline C & 6.04107403 & 0.49300839 & -2.46374147 \\
\hline C & -7.36883992 & -0.99112621 & -4.32893229 \\
\hline $\mathrm{H}$ & -8.25025956 & -1.60544138 & -4.51715398 \\
\hline $\mathrm{H}$ & -6.47907425 & -1.49833629 & -4.72539286 \\
\hline $\mathrm{H}$ & -7.48740399 & -0.02131120 & -4.82966878 \\
\hline C & -5.63929118 & 2.51139631 & 1.49173954 \\
\hline $\mathrm{H}$ & -4.76491499 & 3.05654527 & 1.13168573 \\
\hline $\mathrm{H}$ & -5.73984406 & 2.69909447 & 2.56705903 \\
\hline $\mathrm{H}$ & -6.52915696 & 2.91628357 & 0.99577833 \\
\hline C & -4.31558367 & 0.40660540 & 2.01207342 \\
\hline $\mathrm{H}$ & -4.20867978 & -0.66752288 & 1.81746491 \\
\hline $\mathrm{H}$ & -4.47863191 & 0.53545502 & 3.08855078 \\
\hline $\mathrm{H}$ & -3.38458150 & 0.90559336 & 1.74687988 \\
\hline C & -6.79068991 & 0.31923107 & 1.77476903 \\
\hline $\mathrm{H}$ & -7.69994586 & 0.70665693 & 1.30182524 \\
\hline $\mathrm{H}$ & -6.86926506 & 0.52500875 & 2.84739418 \\
\hline $\mathrm{H}$ & -6.77219860 & -0.76995465 & 1.65151453 \\
\hline C & -0.85042028 & -0.65880575 & 0.30131601 \\
\hline $\mathrm{H}$ & -1.15580911 & -1.70834364 & 0.31049279 \\
\hline $\mathrm{H}$ & -1.69467731 & -0.02397286 & 0.56440014 \\
\hline $\mathrm{H}$ & -0.06935183 & -0.54115985 & 1.05986323 \\
\hline
\end{tabular}

N) Optimized XYZ Coordinates ( $\AA$ ) for CoSal(OAC) adduct $(S=1)$

$\begin{array}{cccc}\text { Co } & 0.02285975 & -0.97192163 & -0.17538085 \\ \text { O } & 1.34205290 & 0.33624273 & -0.41994516 \\ \text { O } & -1.25642473 & 0.38283190 & -0.15177321 \\ \text { O } & 6.85183110 & 0.12462097 & -0.14703271 \\ \text { C } & -3.20024778 & -0.86703905 & -0.79989663 \\ \text { C } & 2.65145971 & 0.24644809 & -0.35455009 \\ \text { O } & -0.78147473 & -3.28114361 & 1.98709906 \\ \text { C } & 4.73864401 & -1.10044774 & -0.24626595 \\ \text { H } & 5.19891508 & -2.08230482 & -0.21564066 \\ \text { C } & 5.48607426 & 0.05483325 & -0.22167544 \\ \text { C } & 3.32282154 & -1.01129182 & -0.30838200 \\ \text { C } & -4.61442031 & -0.92469561 & -0.96060012 \\ \text { H } & -5.05467100 & -1.82834316 & -1.36801742\end{array}$




\begin{tabular}{|c|c|c|c|}
\hline N & -1.16016392 & -2.15898384 & -1.08118325 \\
\hline U & -0.03678547 & -1.15605019 & 1.76106225 \\
\hline C & 1.97064659 & 2.96685823 & -1.73518293 \\
\hline H & 2.61587703 & 2.86132438 & -2.61617589 \\
\hline $\mathrm{H}$ & 1.49863491 & 3.95590363 & -1.78176680 \\
\hline $\mathrm{H}$ & 1.18846060 & 2.20828242 & -1.78495360 \\
\hline C & -0.38939636 & -2.20692961 & 2.45011707 \\
\hline C & 3.45232734 & 1.44627065 & -0.36383267 \\
\hline & 4.82741506 & 1.30350358 & -0.28592337 \\
\hline$\dashv$ & 5.47010670 & 2.17529773 & -0.27149370 \\
\hline V & 1.30011917 & -2.35425438 & -0.43280002 \\
\hline 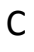 & -2.55350336 & 0.29419425 & -0.26553867 \\
\hline C & 2.58976338 & -2.23096665 & -0.42994863 \\
\hline $\mathrm{H}$ & 3.17803923 & -3.14330368 & -0.56505266 \\
\hline 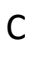 & 3.84852817 & 3.97118786 & -0.44376636 \\
\hline H & 4.52185574 & 3.90563072 & -1.30661616 \\
\hline$H$ & 4.45619883 & 3.98135654 & 0.46856879 \\
\hline $\mathrm{H}$ & 3.33222685 & 4.93571433 & -0.50296982 \\
\hline$c$ & -2.44761894 & -1.99641912 & -1.21304650 \\
\hline $\mathrm{H}$ & -3.00727933 & -2.80965400 & -1.68420280 \\
\hline O & -6.74182833 & 0.24108498 & -0.67658319 \\
\hline C & -5.37864292 & 0.15296894 & -0.58843716 \\
\hline C & -4.74402030 & 1.30146646 & -0.05252445 \\
\hline $\mathrm{H}$ & -5.40475591 & 2.11215305 & 0.22980842 \\
\hline C & 7.56694139 & -1.09533909 & -0.07691789 \\
\hline$H$ & 8.62268011 & -0.82486713 & -0.01395681 \\
\hline$H$ & 7.40414599 & -1.71174106 & -0.97227854 \\
\hline$H$ & 7.28761624 & -1.67663734 & 0.81285941 \\
\hline C & 1.89687575 & 3.05176808 & 0.80474010 \\
\hline $\mathrm{H}$ & 1.42778209 & 4.04241035 & 0.75939353 \\
\hline$H$ & 2.48982067 & 3.00243811 & 1.72604530 \\
\hline H & 1.10987742 & 2.29911117 & 0.86010034 \\
\hline C & -2.75665742 & 2.69200021 & 0.72347019 \\
\hline C & 2.80035933 & 2.84005955 & -0.43407539 \\
\hline C & 0.66564212 & -3.65705527 & -0.66689398 \\
\hline $\mathrm{H}$ & 1.37228945 & -4.36639526 & -1.11358775 \\
\hline $\mathrm{H}$ & 0.29591241 & -4.02497319 & 0.29433962 \\
\hline C & -3.37913863 & 1.41798189 & 0.12270533 \\
\hline C & -0.51878818 & -3.38015481 & -1.58775067 \\
\hline $\mathrm{H}$ & -0.17103785 & -3.20098762 & -2.61448032 \\
\hline $\mathrm{H}$ & -1.22390231 & -4.21916216 & -1.59948451 \\
\hline C & -7.43745495 & -0.88019165 & -1.19070598 \\
\hline $\mathrm{H}$ & -8.49598809 & -0.61405386 & -1.17444565 \\
\hline $\mathrm{H}$ & -7.27773899 & -1.77289931 & -0.57018542 \\
\hline $\mathrm{H}$ & -7.13553213 & -1.10356167 & -2.22358905 \\
\hline C & -1.79797701 & 3.33825694 & -0.30524389 \\
\hline $\mathrm{H}$ & -0.99044775 & 2.65945258 & -0.57941740 \\
\hline $\mathrm{H}$ & -1.35650665 & 4.24887221 & 0.11812260 \\
\hline $\mathrm{H}$ & -2.34190868 & 3.62078170 & -1.21517334 \\
\hline C & -1.99657110 & 2.33884766 & 2.02627094 \\
\hline $\mathrm{H}$ & -2.69233340 & 1.95428833 & 2.78216832 \\
\hline 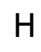 & -1.52301956 & 3.23996437 & 2.43531031 \\
\hline
\end{tabular}




$\begin{array}{lrrr}\text { H } & -1.22452433 & 1.58682720 & 1.85832194 \\ \mathrm{C} & -3.82575035 & 3.74384437 & 1.08255011 \\ \mathrm{H} & -4.38973370 & 4.07943596 & 0.20417546 \\ \mathrm{H} & -3.33125016 & 4.62321671 & 1.50970841 \\ \mathrm{H} & -4.53741869 & 3.37327973 & 1.82957707 \\ \mathrm{C} & -0.29681884 & -1.98826715 & 3.95779005 \\ \mathrm{H} & -0.59893340 & -2.89282969 & 4.48936867 \\ \mathrm{H} & -0.94016024 & -1.15246300 & 4.25241410 \\ \mathrm{H} & 0.72788357 & -1.71786157 & 4.23484552\end{array}$




\section{III.2 TD-DFT excitation energies and oscillator strengths}

A) TD-DFT excitation energies and oscillator strengths for $\mathbf{1}^{+}$

Excited State 1: $3.000-\mathrm{A} \quad 1.5122 \mathrm{eV} 819.89 \mathrm{~nm} \mathrm{f}=0.0000\left\langle\mathrm{~S}^{* *} 2\right\rangle=2.000$

$\begin{array}{ll}137 A->139 A & 0.68207 \\ 138 A->139 A & -0.13168 \\ 137 B->139 B & -0.68207 \\ 138 B->139 B & 0.13168\end{array}$

This state for optimization and/or second-order correction.

Total Energy, E(TD-HF/TD-KS) = -2517.80359811

Copying the excited state density for this state as the 1-particle RhoCl density.

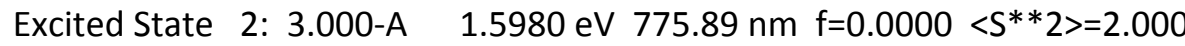

$\begin{array}{lr}137 \mathrm{~A}->139 \mathrm{~A} & 0.13184 \\ 138 \mathrm{~A}->139 \mathrm{~A} & 0.68418 \\ 137 \mathrm{~B}->139 \mathrm{~B} & -0.13184 \\ 138 \mathrm{~B}->139 \mathrm{~B} & -0.68418\end{array}$

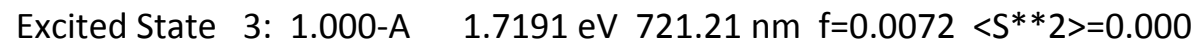
$137 \mathrm{~A} \rightarrow 139 \mathrm{~A} \quad 0.65325$

$138 \mathrm{~A} \rightarrow 139 \mathrm{~A} \quad 0.24517$

$137 \mathrm{~B}->139 \mathrm{~B} \quad 0.65325$

$138 \mathrm{~B}->139 \mathrm{~B} \quad 0.24517$

Excited State 4: $1.000-\mathrm{A} \quad 1.7371 \mathrm{eV} 713.74 \mathrm{~nm} \quad \mathrm{f}=0.0410<\mathrm{S}^{* *} 2>=0.000$

$137 \mathrm{~A}->139 \mathrm{~A} \quad-0.23977$

$138 \mathrm{~A} \rightarrow 139 \mathrm{~A} \quad 0.65391$

$137 \mathrm{~B}->139 \mathrm{~B} \quad-0.23977$

$138 \mathrm{~B}->139 \mathrm{~B} \quad 0.65391$

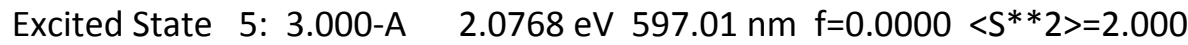
137A $\rightarrow 140 \mathrm{~A}$
0.46978
$138 \mathrm{~A}->140 \mathrm{~A} \quad-0.46168$
$138 \mathrm{~A} \rightarrow 142 \mathrm{~A} \quad-0.12065$
$138 \mathrm{~A} \rightarrow 143 \mathrm{~A} \quad 0.13509$
$137 \mathrm{~B}->140 \mathrm{~B} \quad-0.46978$
$138 \mathrm{~B}->140 \mathrm{~B} \quad 0.46168$
$138 \mathrm{~B}->142 \mathrm{~B} \quad 0.12065$
$138 \mathrm{~B}->143 \mathrm{~B} \quad-0.13509$

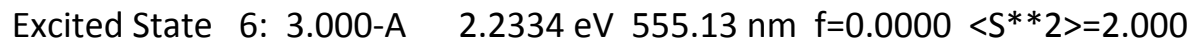
$137 \mathrm{~A} \rightarrow 140 \mathrm{~A}$
0.41207
$137 \mathrm{~A} \rightarrow 142 \mathrm{~A}$
$-0.28598$
$138 \mathrm{~A} \rightarrow>140 \mathrm{~A}$
0.42373
$138 \mathrm{~A} \rightarrow 142 \mathrm{~A}$
$-0.21398$
$137 \mathrm{~B}->140 \mathrm{~B}$
$-0.41207$
$137 \mathrm{~B}->142 \mathrm{~B}$
0.28598
$138 \mathrm{~B}->140 \mathrm{~B}$
$-0.42373$ 


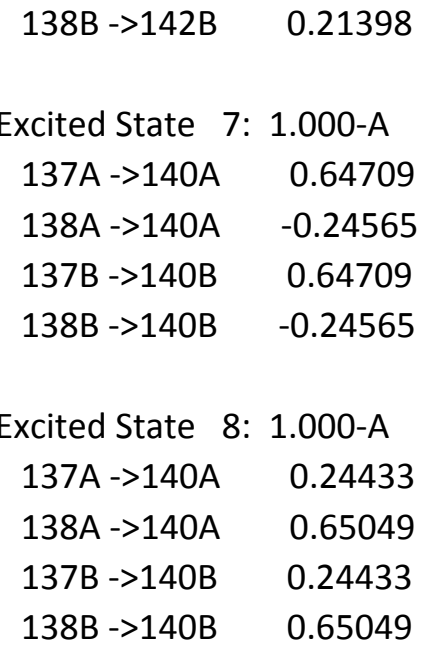
$137 \mathrm{~A} \rightarrow 140 \mathrm{~A} \quad 0.24433$

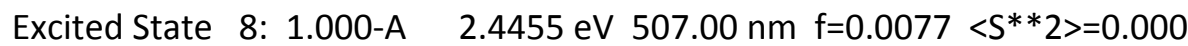

Excited State 9: $3.000-\mathrm{A} \quad 2.5852 \mathrm{eV} \quad 479.59 \mathrm{~nm} \quad \mathrm{f}=0.0000<\mathrm{S}^{* *} 2>=2.000$

$\begin{array}{ll}137 A->140 A & 0.27011 \\ 137 A->142 A & 0.41966 \\ 137 A->143 A & 0.16405 \\ 138 A->140 A & 0.22638 \\ 138 A->142 A & 0.36455 \\ 137 B->140 B & -0.27011 \\ 137 B->142 B & -0.41966 \\ 137 B->143 B & -0.16405 \\ 138 B->140 B & -0.22638 \\ 138 B->142 B & -0.36455\end{array}$

Excited State 10: $3.000-A \quad 2.6510 \mathrm{eV} \quad 467.69 \mathrm{~nm} f=0.0000\left\langle S^{* *} 2>=2.000\right.$ 137A $->141 \mathrm{~A} \quad 0.66929$

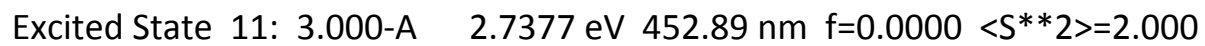
$136 \mathrm{~A}->139 \mathrm{~A} \quad 0.20657$

$137 \mathrm{~A} \rightarrow 141 \mathrm{~A} \quad 0.10265$

$138 \mathrm{~A} \rightarrow 141 \mathrm{~A} \quad 0.63417$

$136 \mathrm{~B}->139 \mathrm{~B} \quad-0.20657$

$137 \mathrm{~B}->141 \mathrm{~B} \quad-0.10265$

$138 \mathrm{~B}->141 \mathrm{~B} \quad-0.63417$

Excited State 12: $3.000-\mathrm{A} \quad 2.8250 \mathrm{eV} \quad 438.88 \mathrm{~nm} f=0.0000\left\langle S^{* *} 2\right\rangle=2.000$ $135 \mathrm{~A} \rightarrow 139 \mathrm{~A} \quad 0.19295$

$136 \mathrm{~A} \rightarrow 139 \mathrm{~A} \quad 0.57369$

136A ->142A 0.14065

$137 \mathrm{~A} \rightarrow 141 \mathrm{~A} \quad-0.10702$

$138 \mathrm{~A} \rightarrow 141 \mathrm{~A} \quad-0.22784$

$138 \mathrm{~A}->142 \mathrm{~A} \quad 0.10108$

$135 B->139 B \quad-0.19295$ 


$\begin{array}{lr}136 \mathrm{~B}->139 \mathrm{~B} & -0.57369 \\ 136 \mathrm{~B}->142 \mathrm{~B} & -0.14065 \\ 137 \mathrm{~B}->141 \mathrm{~B} & 0.10702 \\ 138 \mathrm{~B}->141 \mathrm{~B} & 0.22784 \\ 138 \mathrm{~B}->142 \mathrm{~B} & -0.10108\end{array}$

Excited State 13: $3.000-\mathrm{A} \quad 2.8601 \mathrm{eV} \quad 433.50 \mathrm{~nm} f=0.0000<\mathrm{S}^{* *} 2>=2.000$

$135 \mathrm{~A} \rightarrow 139 \mathrm{~A} \quad-0.20081$

$135 \mathrm{~A} \rightarrow 140 \mathrm{~A} \quad 0.13862$

$136 \mathrm{~A} \rightarrow 139 \mathrm{~A} \quad 0.17295$

$137 A->140 A \quad-0.10283$

$137 \mathrm{~A} \rightarrow 142 \mathrm{~A} \quad 0.18840$

$137 A->143 A \quad-0.31855$

$138 \mathrm{~A}->140 \mathrm{~A} \quad 0.18271$

$138 \mathrm{~A} \rightarrow 142 \mathrm{~A} \quad-0.19212$

$138 \mathrm{~A} \rightarrow 143 \mathrm{~A} \quad 0.38179$

$135 B->139 B \quad 0.20081$

$135 \mathrm{~B}->140 \mathrm{~B} \quad-0.13862$

$136 B->139 B \quad-0.17295$

$137 \mathrm{~B}->140 \mathrm{~B} \quad 0.10283$

$137 B->142 B \quad-0.18840$

$137 \mathrm{~B}->143 \mathrm{~B} \quad 0.31855$

$138 \mathrm{~B}->140 \mathrm{~B} \quad-0.18271$

$138 \mathrm{~B}->142 \mathrm{~B} \quad 0.19212$

$138 \mathrm{~B}->143 \mathrm{~B} \quad-0.38179$

Excited State 14: $1.000-\mathrm{A} \quad 2.8698 \mathrm{eV} \quad 432.03 \mathrm{~nm} f=0.0643\left\langle\mathrm{~S}^{* *} 2>=0.000\right.$ $136 \mathrm{~A} \rightarrow 139 \mathrm{~A} \quad 0.15684$

$137 A->141 A \quad 0.29445$

$138 \mathrm{~A}->141 \mathrm{~A} \quad 0.61058$

$136 \mathrm{~B}->139 \mathrm{~B} \quad 0.15684$

137B $->141 B \quad 0.29445$

$138 \mathrm{~B}->141 \mathrm{~B} \quad 0.61058$

Excited State 15: $1.000-\mathrm{A} \quad 2.9077 \mathrm{eV} \quad 426.39 \mathrm{~nm} \mathrm{f}=0.0407\left\langle\mathrm{~S}^{* *} 2\right\rangle=0.000$ $135 \mathrm{~A} \rightarrow 139 \mathrm{~A} \quad 0.13752$

$136 \mathrm{~A}->139 \mathrm{~A} \quad 0.37903$

$137 \mathrm{~A}->141 \mathrm{~A} \quad 0.45996$

$138 \mathrm{~A}->141 \mathrm{~A} \quad-0.32939$

$135 B->139 B \quad 0.13752$

$136 \mathrm{~B}->139 \mathrm{~B} \quad 0.37903$

$137 \mathrm{~B}->141 \mathrm{~B} \quad 0.45996$

$138 \mathrm{~B}->141 \mathrm{~B} \quad-0.32939$

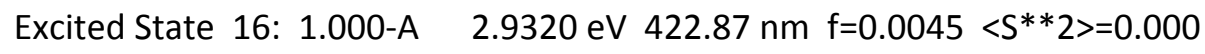

$136 \mathrm{~A} \rightarrow 139 \mathrm{~A} \quad 0.56610$

$137 A->141 A \quad-0.39247$

$136 \mathrm{~B}->139 \mathrm{~B} \quad 0.56610$ 


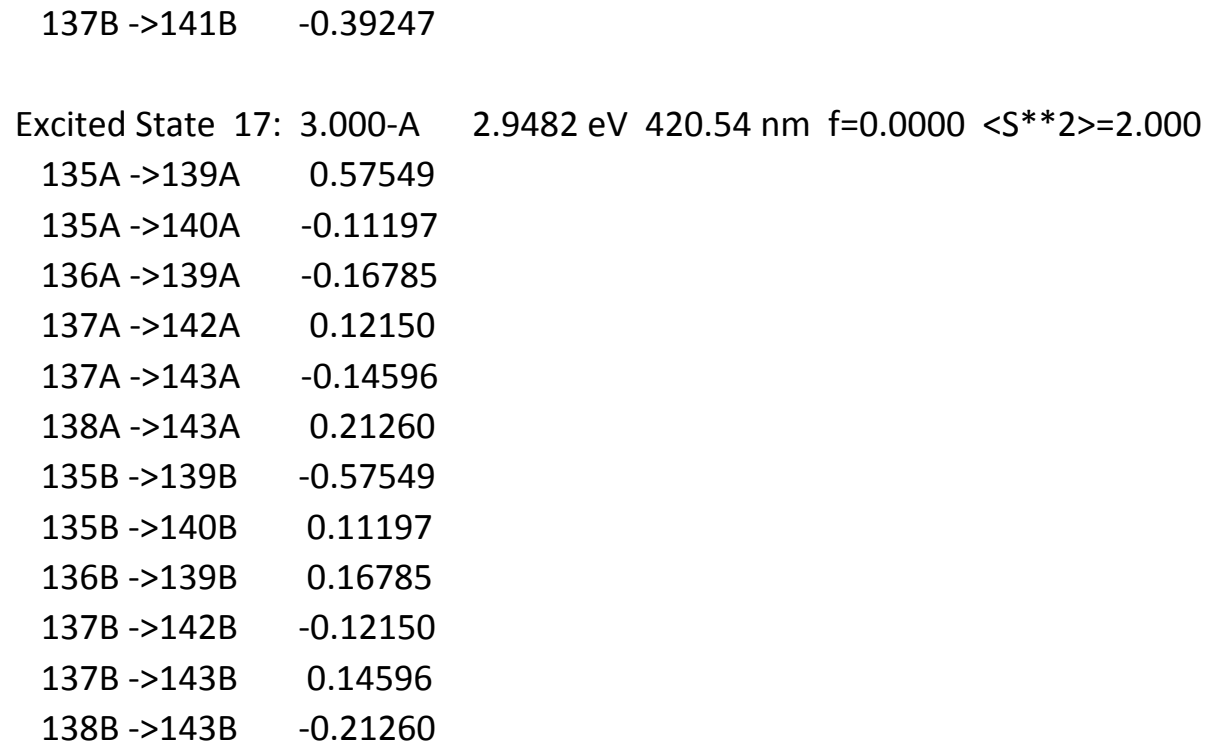

Excited State 18: $1.000-\mathrm{A} \quad 3.0193 \mathrm{eV} \quad 410.65 \mathrm{~nm} \mathrm{f}=0.0022\left\langle\mathrm{~S}^{* *} 2\right\rangle=0.000$ $135 \mathrm{~A} \rightarrow 139 \mathrm{~A} \quad 0.67823$

Excited State 19: $3.000-A \quad 3.1174 \mathrm{eV} \quad 397.72 \mathrm{~nm} f=0.0000\left\langle S^{* *} 2\right\rangle=2.000$ $135 \mathrm{~A}->139 \mathrm{~A} \quad 0.21760$

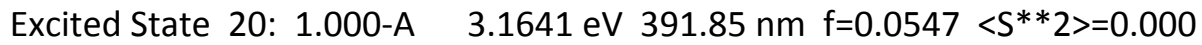
$137 \mathrm{~A} \rightarrow 142 \mathrm{~A} \quad 0.53678$

$137 \mathrm{~A} \rightarrow 143 \mathrm{~A} \quad 0.10313$

$138 \mathrm{~A}->142 \mathrm{~A} \quad 0.41252$

$137 \mathrm{~B}->142 \mathrm{~B} \quad 0.53678$

$137 B->143 B \quad 0.10313$

$138 \mathrm{~B}->142 \mathrm{~B} \quad 0.41252$ 


$\begin{array}{cc}\text { Excited State } 21: & 3.000-A \\ 133 A->139 A & -0.23833 \\ 135 A->139 A & -0.11357 \\ 135 A->140 A & -0.18270 \\ 135 A->142 A & 0.17278 \\ 136 A->139 A & -0.15224 \\ 136 A->140 A & -0.27909 \\ 136 A->142 A & 0.36569 \\ 138 A->142 A & 0.14425 \\ 138 A->143 A & 0.10120 \\ 133 B->139 B & 0.23833 \\ 135 B->139 B & 0.11357 \\ 135 B->140 B & 0.18270 \\ 135 B->142 B & -0.17278 \\ 136 B->139 B & 0.15224 \\ 136 B->140 B & 0.27909 \\ 136 B->142 B & -0.36569 \\ 138 B->142 B & -0.14425 \\ 138 B->143 B & -0.10120\end{array}$

Excited State 22: 1.000-A $137 \mathrm{~A} \rightarrow 142 \mathrm{~A} \quad-0.42283$ $138 \mathrm{~A} \rightarrow 142 \mathrm{~A} \quad 0.54884$ $137 \mathrm{~B}->142 B \quad-0.42283$ $138 \mathrm{~B}->142 \mathrm{~B} \quad 0.54884$

Excited State 23: 3.000-A 126A $>$ 139A $\quad-0.10116$ $132 A->139 A \quad 0.13232$ $133 \mathrm{~A}->139 \mathrm{~A} \quad 0.35034$ $134 \mathrm{~A} \rightarrow 139 \mathrm{~A} \quad 0.13815$ $137 \mathrm{~A}->142 \mathrm{~A} \quad-0.27509$ $137 A->143 A \quad-0.16884$ $138 \mathrm{~A}->142 \mathrm{~A} \quad 0.35599$ $138 \mathrm{~A} \rightarrow 143 \mathrm{~A} \quad 0.19730$ $126 \mathrm{~B}->139 \mathrm{~B} \quad 0.10116$ $132 B->139 B \quad-0.13232$ $133 B->139 B \quad-0.35034$ $134 B->139 B \quad-0.13815$ 137B $->142 B \quad 0.27509$ $137 \mathrm{~B}->143 B \quad 0.16884$ $138 \mathrm{~B}->142 \mathrm{~B} \quad-0.35599$ $138 \mathrm{~B}->143 \mathrm{~B} \quad-0.19730$

Excited State 24: 3.000-A 132A $\rightarrow 139 A$ 0.11793

$133 A->139 A$ 0.35728

$134 A->139 A$ 0.13104

3.2019 eV $\left.387.23 \mathrm{~nm} \mathrm{f}=0.0000<S^{* *} 2\right\rangle=2.000$

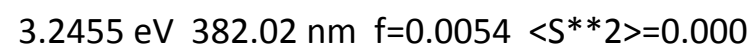

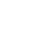




$$
\begin{array}{ll}
135 \mathrm{~A}->139 \mathrm{~A} & -0.11930 \\
135 \mathrm{~A}->140 \mathrm{~A} & -0.12097 \\
136 \mathrm{~A}->139 \mathrm{~A} & -0.10774 \\
136 \mathrm{~A}->140 \mathrm{~A} & -0.11865 \\
136 \mathrm{~A}->142 \mathrm{~A} & 0.18689 \\
137 \mathrm{~A}->142 \mathrm{~A} & 0.26085 \\
137 \mathrm{~A}->143 \mathrm{~A} & 0.14611 \\
138 \mathrm{~A}->142 \mathrm{~A} & -0.26334 \\
138 \mathrm{~A}->143 \mathrm{~A} & -0.12275 \\
132 \mathrm{~B}->139 \mathrm{~B} & -0.11793 \\
133 \mathrm{~B}->139 \mathrm{~B} & -0.35728 \\
134 \mathrm{~B}->139 \mathrm{~B} & -0.13104 \\
135 \mathrm{~B}->139 \mathrm{~B} & 0.11930 \\
135 \mathrm{~B}->140 \mathrm{~B} & 0.12097 \\
136 \mathrm{~B}->139 \mathrm{~B} & 0.10774 \\
136 \mathrm{~B}->140 \mathrm{~B} & 0.11865 \\
136 \mathrm{~B}->142 \mathrm{~B} & -0.18689 \\
137 \mathrm{~B}->142 \mathrm{~B} & -0.26085 \\
137 \mathrm{~B}->143 \mathrm{~B} & -0.14611 \\
138 \mathrm{~B}->142 \mathrm{~B} & 0.26334 \\
138 \mathrm{~B}->143 \mathrm{~B} & 0.12275
\end{array}
$$

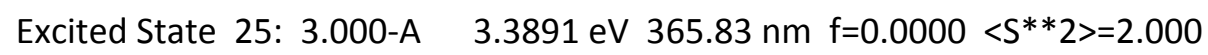

$$
\begin{array}{ll}
112 A->139 A & 0.12975 \\
120 A->139 A & -0.19377 \\
121 A->139 A & -0.13869 \\
124 A->139 A & -0.10278 \\
131 A->139 A & 0.47572 \\
131 A->140 A & 0.10146 \\
134 A->139 A & 0.17188 \\
112 B->139 B & -0.12975 \\
120 B->139 B & 0.19377 \\
121 B->139 B & 0.13869 \\
124 B->139 B & 0.10278 \\
131 B->139 B & -0.47572 \\
131 B->140 B & -0.10146 \\
134 B->139 B & -0.17188
\end{array}
$$

Excited State 26: $1.000-\mathrm{A} \quad 3.4652 \mathrm{eV} \quad 357.80 \mathrm{~nm} f=0.0420\left\langle S^{* *} 2>=0.000\right.$

$$
\begin{array}{cr}
135 A->140 A & -0.14747 \\
136 A->140 A & 0.11573 \\
137 A->143 A & -0.44015 \\
138 A->143 A & 0.47569 \\
135 B->140 B & -0.14747 \\
136 B->140 B & 0.11573 \\
137 B->143 B & -0.44015 \\
138 B->143 B & 0.47569
\end{array}
$$


$\begin{array}{cc}\text { Excited State } 27: & 3.000-A \\ 137 A->142 A & -0.13232 \\ 137 A->143 A & 0.51121 \\ 138 A->143 A & 0.43105 \\ 137 B->142 B & 0.13232 \\ 137 B->143 B & -0.51121 \\ 138 B->143 B & -0.43105\end{array}$

Excited State 28: $1.000-\mathrm{A} \quad 3.5708 \mathrm{eV} \quad 347.22 \mathrm{~nm} \mathrm{f}=0.0014\left\langle\mathrm{~S}^{* *} 2\right\rangle=0.000$ $137 \mathrm{~A} \rightarrow 143 \mathrm{~A} \quad 0.48076$ $138 \mathrm{~A} \rightarrow 143 \mathrm{~A} \quad 0.47555$ $137 B->143 B \quad 0.48076$ $138 \mathrm{~B}->143 \mathrm{~B} \quad 0.47555$

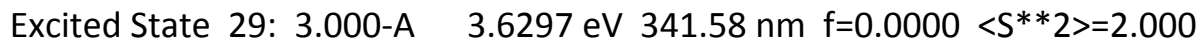
$\begin{array}{ll}131 \mathrm{~A}->139 \mathrm{~A} & -0.14807 \\ 135 \mathrm{~A}->140 \mathrm{~A} & 0.25961 \\ 135 \mathrm{~A}->142 \mathrm{~A} & 0.10972 \\ 136 \mathrm{~A}->140 \mathrm{~A} & 0.40143 \\ 136 \mathrm{~A}->142 \mathrm{~A} & 0.23497 \\ 137 \mathrm{~A}->144 \mathrm{~A} & -0.11188 \\ 138 \mathrm{~A}->144 \mathrm{~A} & -0.10154 \\ 131 \mathrm{~B}->139 \mathrm{~B} & 0.14807 \\ 135 \mathrm{~B}->140 \mathrm{~B} & -0.25961 \\ 135 \mathrm{~B}->142 \mathrm{~B} & -0.10972 \\ 136 \mathrm{~B}->140 \mathrm{~B} & -0.40143 \\ 136 \mathrm{~B}->142 \mathrm{~B} & -0.23497 \\ 137 \mathrm{~B}->144 \mathrm{~B} & 0.11188 \\ 138 \mathrm{~B}->144 \mathrm{~B} & 0.10154\end{array}$

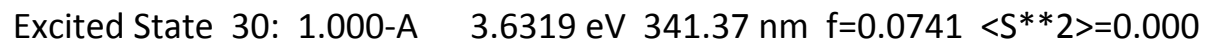
$136 \mathrm{~A} \rightarrow 140 \mathrm{~A} \quad 0.67215$

$137 A->143 A \quad 0.13660$

$136 \mathrm{~B}->140 \mathrm{~B} \quad 0.67215$

$137 \mathrm{~B}->143 \mathrm{~B} \quad 0.13660$

B) TD-DFT excitation energies and oscillator strengths for $\mathbf{2}^{+}$

Excited State 1: $3.000-\mathrm{A} \quad 1.4383 \mathrm{eV} 862.00 \mathrm{~nm} \mathrm{f}=0.0000\left\langle\mathrm{~S}^{* *} 2\right\rangle=2.000$
$141 \mathrm{~A} \rightarrow 143 \mathrm{~A}$
0.53512
$142 \mathrm{~A}->143 \mathrm{~A}$
$-0.44518$
$141 B->143 B \quad-0.53512$
$142 B->143 B \quad 0.44518$

This state for optimization and/or second-order correction.

Total Energy, E(TD-HF/TD-KS) = -2557.12356518

Copying the excited state density for this state as the 1-particle RhoCl density.

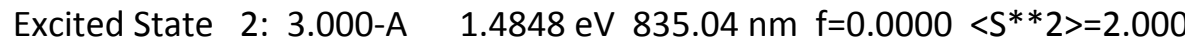
$141 \mathrm{~A} \rightarrow 143 \mathrm{~A} \quad 0.44378$

$142 \mathrm{~A} \rightarrow 143 \mathrm{~A} \quad 0.53793$ 


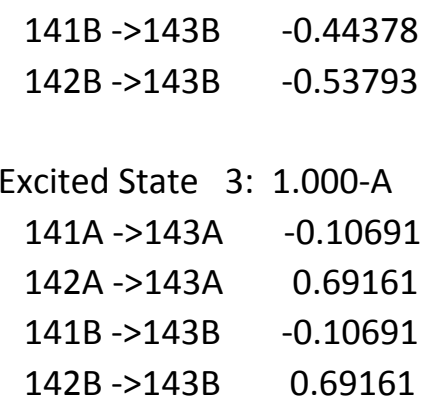
141A $->143 A \quad-0.10691$

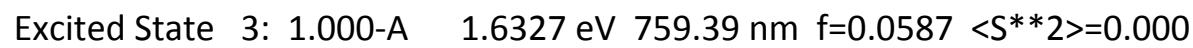

Excited State $4: 1.000-\mathrm{A} \quad 1.7418 \mathrm{eV} 711.82 \mathrm{~nm} \mathrm{f}=0.0162\left\langle\mathrm{~S}^{* *} 2>=0.000\right.$ $141 \mathrm{~A} \rightarrow 143 \mathrm{~A} \quad 0.68479$

$142 \mathrm{~A} \rightarrow 143 \mathrm{~A} \quad 0.10620$

$141 B->143 B \quad 0.68479$

$142 B->143 B \quad 0.10620$

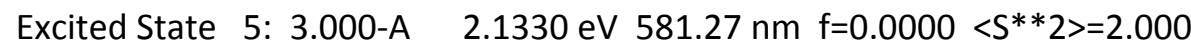
$141 \mathrm{~A} \rightarrow 144 \mathrm{~A} \quad 0.48438$

$141 \mathrm{~A} \rightarrow 146 \mathrm{~A} \quad-0.15918$

$141 \mathrm{~A} \rightarrow>147 \mathrm{~A} \quad-0.11282$

$142 \mathrm{~A} \rightarrow 144 \mathrm{~A} \quad 0.43447$

$141 \mathrm{~B}->144 \mathrm{~B} \quad-0.48438$

$141 \mathrm{~B}->146 \mathrm{~B} \quad 0.15918$

$141 \mathrm{~B}->147 \mathrm{~B} \quad 0.11282$

$142 B->144 B \quad-0.43447$

Excited State 6: $3.000-\mathrm{A} \quad 2.2083 \mathrm{eV} 561.45 \mathrm{~nm} \quad \mathrm{f}=0.0000<\mathrm{S}^{* *} 2>=2.000$ $141 \mathrm{~A} \rightarrow 144 \mathrm{~A} \quad 0.41598$

$141 \mathrm{~A} \rightarrow 146 \mathrm{~A} \quad 0.11471$

$142 \mathrm{~A} \rightarrow 144 \mathrm{~A} \quad-0.46660$

$142 \mathrm{~A}->146 \mathrm{~A} \quad-0.27926$

$141 \mathrm{~B}->144 \mathrm{~B} \quad-0.41598$

$141 \mathrm{~B}->146 \mathrm{~B} \quad-0.11471$

$142 B->144 B \quad 0.46660$

$142 B->146 B \quad 0.27926$

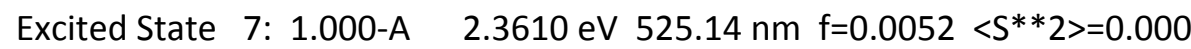
$141 \mathrm{~A} \rightarrow 144 \mathrm{~A} \quad-0.16126$

$142 \mathrm{~A} \rightarrow 144 \mathrm{~A} \quad 0.67561$

$141 B->144 B \quad-0.16126$

$142 B->144 B \quad 0.67561$

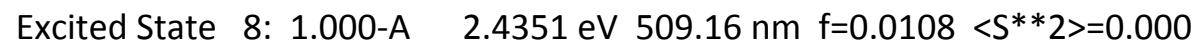
$\begin{array}{ll}141 A->144 A & 0.67175 \\ 142 A->144 A & 0.16096 \\ 141 B->144 B & 0.67175 \\ 142 B->144 B & 0.16096\end{array}$

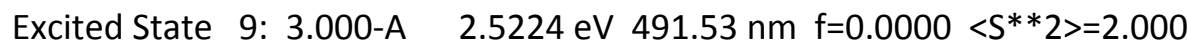




$$
\begin{array}{lr}
141 \mathrm{~A}->144 \mathrm{~A} & -0.13038 \\
141 \mathrm{~A}->145 \mathrm{~A} & -0.25626 \\
141 \mathrm{~A}->146 \mathrm{~A} & 0.19950 \\
142 \mathrm{~A}->144 \mathrm{~A} & 0.17559 \\
142 \mathrm{~A}->145 \mathrm{~A} & 0.37315 \\
142 \mathrm{~A}->146 \mathrm{~A} & -0.40424 \\
142 \mathrm{~A}->147 \mathrm{~A} & 0.13547 \\
141 \mathrm{~B}->144 \mathrm{~B} & 0.13038 \\
141 \mathrm{~B}->145 \mathrm{~B} & 0.25626 \\
141 \mathrm{~B}->146 \mathrm{~B} & -0.19950 \\
142 \mathrm{~B}->144 \mathrm{~B} & -0.17559 \\
142 \mathrm{~B}->145 \mathrm{~B} & -0.37315 \\
142 \mathrm{~B}->146 \mathrm{~B} & 0.40424 \\
142 \mathrm{~B}->147 \mathrm{~B} & -0.13547
\end{array}
$$

Excited State 10: $3.000-A \quad 2.6169 \mathrm{eV} \quad 473.78 \mathrm{~nm} f=0.0000\left\langle S^{* *} 2>=2.000\right.$

$$
\begin{array}{lr}
141 \mathrm{~A} \rightarrow>144 \mathrm{~A} & 0.11297 \\
141 \mathrm{~A}->145 \mathrm{~A} & 0.58502 \\
142 \mathrm{~A}->144 \mathrm{~A} & 0.11040 \\
142 \mathrm{~A}->145 \mathrm{~A} & 0.31494 \\
141 \mathrm{~B}->144 \mathrm{~B} & -0.11297 \\
141 \mathrm{~B}->145 \mathrm{~B} & -0.58502 \\
142 \mathrm{~B}->144 \mathrm{~B} & -0.11040 \\
142 \mathrm{~B}->145 \mathrm{~B} & -0.31494
\end{array}
$$

Excited State 11: 3.000-A $2.6274 \mathrm{eV} \quad 471.89 \mathrm{~nm} \mathrm{f=0.0000}\left\langle\mathrm{S}^{* *} 2>=2.000\right.$

$$
\begin{array}{lr}
140 \mathrm{~A}->143 \mathrm{~A} & 0.17023 \\
140 \mathrm{~A}->146 \mathrm{~A} & 0.11717 \\
141 \mathrm{~A}->144 \mathrm{~A} & -0.14348 \\
141 \mathrm{~A}->145 \mathrm{~A} & 0.21084 \\
141 \mathrm{~A}->146 \mathrm{~A} & 0.11867 \\
141 \mathrm{~A}->147 \mathrm{~A} & -0.12508 \\
142 \mathrm{~A}->144 \mathrm{~A} & 0.14675 \\
142 \mathrm{~A}->145 \mathrm{~A} & -0.46080 \\
142 \mathrm{~A}->146 \mathrm{~A} & -0.29706 \\
142 \mathrm{~A}->147 \mathrm{~A} & 0.12507 \\
140 \mathrm{~B}->143 \mathrm{~B} & -0.17023 \\
140 \mathrm{~B}->146 \mathrm{~B} & -0.11717 \\
141 \mathrm{~B}->144 \mathrm{~B} & 0.14348 \\
141 \mathrm{~B}->145 \mathrm{~B} & -0.21084 \\
141 \mathrm{~B}->146 \mathrm{~B} & -0.11867 \\
141 \mathrm{~B}->147 \mathrm{~B} & 0.12508 \\
142 \mathrm{~B}->144 \mathrm{~B} & -0.14675 \\
142 \mathrm{~B}->145 \mathrm{~B} & 0.46080 \\
142 \mathrm{~B}->146 \mathrm{~B} & 0.29706 \\
142 \mathrm{~B}->147 \mathrm{~B} & -0.12507
\end{array}
$$

Excited State 12: $1.000-A \quad 2.7370 \mathrm{eV} \quad 452.99 \mathrm{~nm} f=0.0726\left\langle S^{* *} 2>=0.000\right.$ 


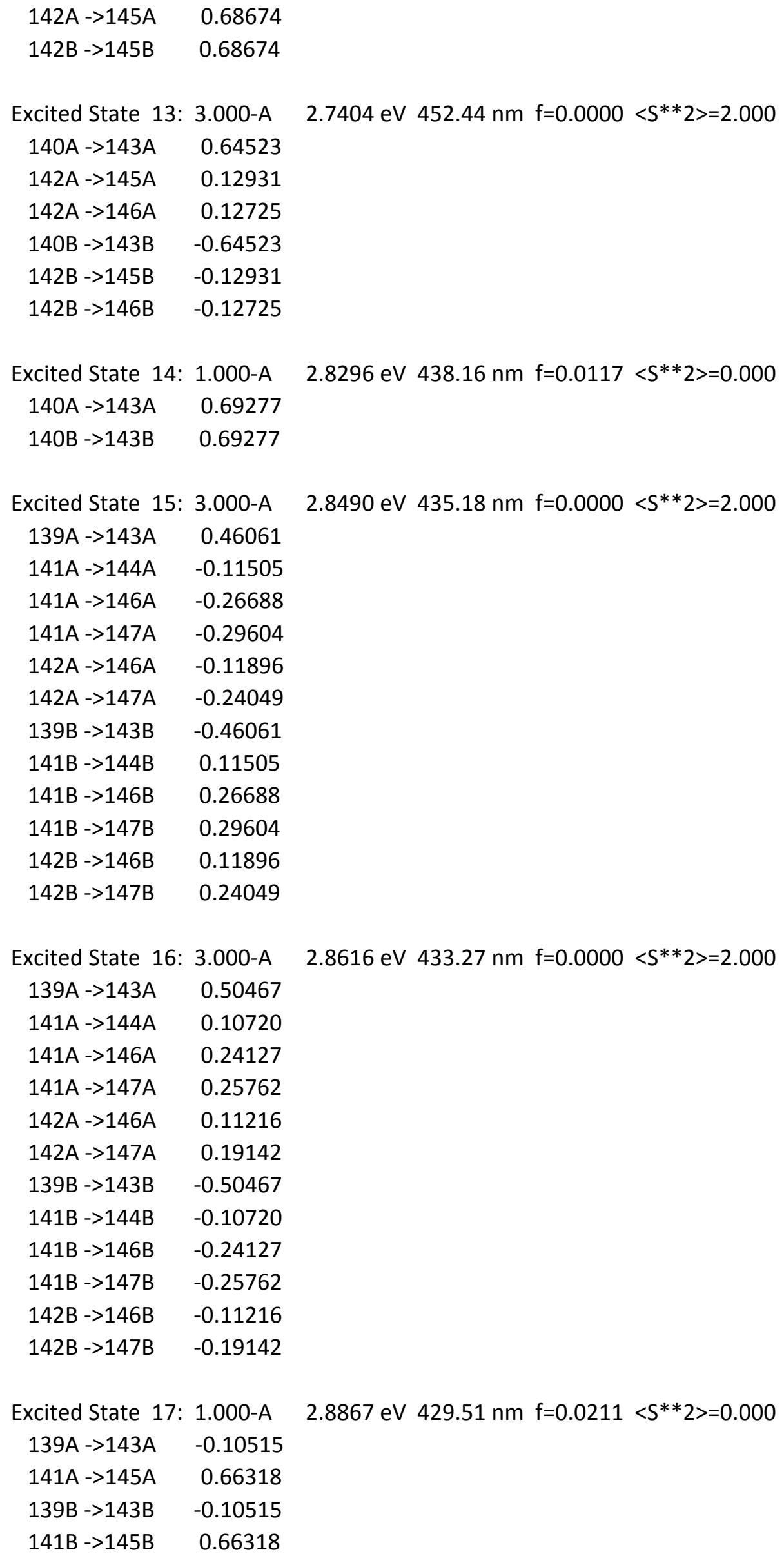

Excited State 16: $3.000-\mathrm{A} \quad 2.8616 \mathrm{eV} \quad 433.27 \mathrm{~nm} f=0.0000\left\langle S^{* *} 2>=2.000\right.$ $139 \mathrm{~A} \rightarrow 143 \mathrm{~A} \quad 0.50467$

Excited State 17: $1.000-\mathrm{A} \quad 2.8867 \mathrm{eV} \quad 429.51 \mathrm{~nm} \mathrm{f}=0.0211\left\langle S^{* *} 2\right\rangle=0.000$ $139 \mathrm{~A} \rightarrow 143 \mathrm{~A} \quad-0.10515$ 
$\begin{array}{cc}\text { Excited State } 18: & 1.000-A \\ 139 A->143 A & 0.69169 \\ 141 A->145 A & 0.10765 \\ 139 B->143 B & 0.69169 \\ 141 B->145 B & 0.10765\end{array}$

Excited State 19: $3.000-\mathrm{A} \quad 3.1037 \mathrm{eV} \quad 399.48 \mathrm{~nm} \quad \mathrm{f}=0.0000<S^{* *} 2>=2.000$ 139A $>144 \mathrm{~A} \quad-0.50114$ $139 A->146 A \quad 0.23474$ $139 A->147 A \quad 0.29240$ $141 \mathrm{~A} \rightarrow 150 \mathrm{~A} \quad-0.11096$ $139 \mathrm{~B}->144 \mathrm{~B} \quad 0.50114$ $139 B->146 B \quad-0.23474$ $139 B->147 B \quad-0.29240$ $141 \mathrm{~B}->150 \mathrm{~B} \quad 0.11096$

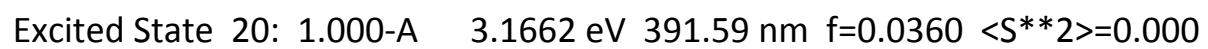
$141 \mathrm{~A} \rightarrow 146 \mathrm{~A} \quad-0.14213$ $142 \mathrm{~A} \rightarrow 146 \mathrm{~A} \quad 0.66438$ $141 \mathrm{~B}->146 \mathrm{~B} \quad-0.14213$ $142 B->146 B \quad 0.66438$

Excited State 21: $3.000-\mathrm{A} \quad 3.1735 \mathrm{eV} 390.68 \mathrm{~nm} f=0.0000<\mathrm{S}^{* *} 2>=2.000$ $135 \mathrm{~A}->143 \mathrm{~A} \quad 0.10390$ $136 \mathrm{~A} \rightarrow 143 \mathrm{~A} \quad 0.14718$ $137 \mathrm{~A} \rightarrow 143 \mathrm{~A} \quad-0.21710$ $140 \mathrm{~A} \rightarrow 144 \mathrm{~A} \quad 0.33385$ $140 \mathrm{~A}->146 \mathrm{~A} \quad 0.42324$ $140 \mathrm{~A} \rightarrow 147 \mathrm{~A} \quad-0.11335$ $140 \mathrm{~A} \rightarrow 148 \mathrm{~A} \quad-0.10193$ $141 \mathrm{~A} \rightarrow 146 \mathrm{~A} \quad-0.10897$ $135 B->143 B \quad-0.10390$ $136 B->143 B \quad-0.14718$ $137 \mathrm{~B}->143 \mathrm{~B} \quad 0.21710$ $140 \mathrm{~B}->144 \mathrm{~B} \quad-0.33385$ $140 \mathrm{~B}->146 \mathrm{~B} \quad-0.42324$ $140 \mathrm{~B}->147 \mathrm{~B} \quad 0.11335$ $140 \mathrm{~B}->148 \mathrm{~B} \quad 0.10193$ $141 \mathrm{~B}->146 \mathrm{~B} \quad 0.10897$

Excited State 22: $3.000-\mathrm{A} \quad 3.2079 \mathrm{eV} \quad 386.50 \mathrm{~nm} f=0.0000\left\langle\mathrm{~S}^{* *} 2>=2.000\right.$ $125 \mathrm{~A} \rightarrow 143 \mathrm{~A} \quad-0.10357$ $128 \mathrm{~A}->143 \mathrm{~A} \quad 0.13975$ $134 \mathrm{~A} \rightarrow 143 \mathrm{~A} \quad 0.14952$ $136 \mathrm{~A} \rightarrow 143 \mathrm{~A} \quad-0.32647$ $137 A->143 A \quad 0.43863$ $138 \mathrm{~A} \rightarrow 143 \mathrm{~A} \quad 0.10371$

$2.9147 \mathrm{eV} 425.37 \mathrm{~nm} \mathrm{f}=0.0014\left\langle\mathrm{~S}^{* *} 2\right\rangle=0.000$ 


$$
\begin{array}{lr}
140 \mathrm{~A}->143 \mathrm{~A} & -0.15684 \\
140 \mathrm{~A}->144 \mathrm{~A} & 0.14793 \\
140 \mathrm{~A}->146 \mathrm{~A} & 0.18913 \\
125 \mathrm{~B}->143 \mathrm{~B} & 0.10357 \\
128 \mathrm{~B}->143 \mathrm{~B} & -0.13975 \\
134 \mathrm{~B}->143 \mathrm{~B} & -0.14952 \\
136 \mathrm{~B}->143 \mathrm{~B} & 0.32647 \\
137 \mathrm{~B}->143 \mathrm{~B} & -0.43863 \\
138 \mathrm{~B}->143 \mathrm{~B} & -0.10371 \\
140 \mathrm{~B}->143 \mathrm{~B} & 0.15684 \\
140 \mathrm{~B}->144 \mathrm{~B} & -0.14793 \\
140 \mathrm{~B}->146 \mathrm{~B} & -0.18913
\end{array}
$$

Excited State 23: $3.000-\mathrm{A} \quad 3.2364 \mathrm{eV} \quad 383.09 \mathrm{~nm} \mathrm{f}=0.0000\left\langle\mathrm{~S}^{* *} 2\right\rangle=2.000$

$$
\begin{array}{ll}
115 A->143 A & -0.14366 \\
135 A->143 A & 0.39027 \\
138 A->143 A & 0.38850 \\
139 A->143 A & 0.14045 \\
140 A->143 A & 0.10315 \\
115 B->143 B & 0.14366 \\
135 B->143 B & -0.39027 \\
138 B->143 B & -0.38850 \\
139 B->143 B & -0.14045 \\
140 B->143 B & -0.10315
\end{array}
$$

Excited State 24: $1.000-\mathrm{A} \quad 3.3151 \mathrm{eV} \quad 374.00 \mathrm{~nm} f=0.0363\left\langle\mathrm{~S}^{* *} 2\right\rangle=0.000$

$$
\begin{array}{ll}
141 \mathrm{~A}->146 \mathrm{~A} & 0.66594 \\
142 \mathrm{~A}->146 \mathrm{~A} & 0.15250 \\
141 \mathrm{~B}->146 \mathrm{~B} & 0.66594 \\
142 \mathrm{~B}->146 \mathrm{~B} & 0.15250
\end{array}
$$

Excited State 25: 3.000-A $3.3652 \mathrm{eV} 368.43 \mathrm{~nm} f=0.0000<\mathrm{S}^{* *} 2>=2.000$

$$
\begin{array}{lr}
141 \mathrm{~A}->146 \mathrm{~A} & 0.44870 \\
141 \mathrm{~A}->147 \mathrm{~A} & -0.36598 \\
141 \mathrm{~A}->148 \mathrm{~A} & 0.12513 \\
142 \mathrm{~A}->146 \mathrm{~A} & 0.24185 \\
142 \mathrm{~A}->147 \mathrm{~A} & -0.23952 \\
141 \mathrm{~B}->146 \mathrm{~B} & -0.44870 \\
141 \mathrm{~B}->147 \mathrm{~B} & 0.36598 \\
141 \mathrm{~B}->148 \mathrm{~B} & -0.12513 \\
142 \mathrm{~B}->146 \mathrm{~B} & -0.24185 \\
142 \mathrm{~B}->147 \mathrm{~B} & 0.23952
\end{array}
$$

Excited State 26: $1.000-\mathrm{A} \quad 3.4844 \mathrm{eV} 355.83 \mathrm{~nm} f=0.0279\left\langle S^{* *} 2\right\rangle=0.000$

$$
\begin{array}{lr}
139 A->144 A & 0.13444 \\
141 A->146 A & -0.10403 \\
141 A->147 A & 0.28508 \\
142 A->147 A & 0.59332
\end{array}
$$




$$
\begin{array}{lr}
139 B->144 B & 0.13444 \\
141 B->146 B & -0.10403 \\
141 B->147 B & 0.28508 \\
142 B->147 B & 0.59332
\end{array}
$$

Excited State 27: $3.000-\mathrm{A} \quad 3.4972 \mathrm{eV} 354.52 \mathrm{~nm} f=0.0000\left\langle S^{* *} 2\right\rangle=2.000$

$$
141 \mathrm{~A} \rightarrow 146 \mathrm{~A} \quad-0.13203
$$$$
141 \mathrm{~A}->147 \mathrm{~A} \quad-0.37394
$$$$
142 \mathrm{~A}->146 \mathrm{~A} \quad 0.18602
$$$$
142 \mathrm{~A} \rightarrow 147 \mathrm{~A} \quad 0.52649
$$$$
141 B->146 B \quad 0.13203
$$$$
141 B->147 B \quad 0.37394
$$$$
142 B->146 B \quad-0.18602
$$$$
142 B->147 B \quad-0.52649
$$

Excited State 28: $1.000-\mathrm{A}$ 139A $\rightarrow 144 \mathrm{~A} \quad 0.12901$ $140 \mathrm{~A} \rightarrow 144 \mathrm{~A} \quad 0.12451$ $141 \mathrm{~A}->147 \mathrm{~A} \quad 0.56864$ $142 \mathrm{~A}->146 \mathrm{~A} \quad-0.12252$ $142 \mathrm{~A} \rightarrow 147 \mathrm{~A} \quad-0.30261$ $139 B->144 B \quad 0.12901$ $140 \mathrm{~B}->144 \mathrm{~B} \quad 0.12451$ $141 B->147 B \quad 0.56864$ $142 B->146 B \quad-0.12252$ $142 B->147 B \quad-0.30261$

Excited State 29: $3.000-\mathrm{A} \quad 3.5725 \mathrm{eV} 347.06 \mathrm{~nm} f=0.0000<\mathrm{S}^{* *} 2>=2.000$ $112 \mathrm{~A} \rightarrow 144 \mathrm{~A} \quad-0.10413$ $138 \mathrm{~A} \rightarrow 143 \mathrm{~A} \quad 0.11343$ $140 \mathrm{~A} \rightarrow 144 \mathrm{~A} \quad 0.48905$ $140 \mathrm{~A} \rightarrow 145 \mathrm{~A} \quad 0.14456$ $140 \mathrm{~A} \rightarrow 146 \mathrm{~A} \quad-0.24022$ $140 \mathrm{~A}->147 \mathrm{~A} \quad 0.14906$ $142 \mathrm{~A} \rightarrow 148 \mathrm{~A} \quad 0.10495$ $142 \mathrm{~A} \rightarrow 151 \mathrm{~A} \quad 0.10450$ $112 B->144 B \quad 0.10413$ $138 \mathrm{~B}->143 \mathrm{~B} \quad-0.11343$ $140 \mathrm{~B}->144 \mathrm{~B} \quad-0.48905$ $140 \mathrm{~B}->145 \mathrm{~B} \quad-0.14456$ $140 \mathrm{~B}->146 \mathrm{~B} \quad 0.24022$ $140 B->147 B \quad-0.14906$ $142 B->148 B \quad-0.10495$ $142 B->151 B \quad-0.10450$

Excited State 30: $1.000-\mathrm{A} \quad 3.5817 \mathrm{eV} \quad 346.16 \mathrm{~nm} f=0.0148\left\langle\mathrm{~S}^{* *} 2>=0.000\right.$ $140 \mathrm{~A} \rightarrow 144 \mathrm{~A} \quad 0.67137$ $141 \mathrm{~A} \rightarrow 147 \mathrm{~A} \quad-0.11808$

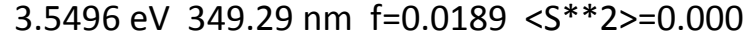
. 
$\begin{array}{ll}140 \mathrm{~B}->144 \mathrm{~B} & 0.67137 \\ 141 \mathrm{~B}->147 \mathrm{~B} & -0.11808\end{array}$

C) TD-DFT excitation energies and oscillator strengths for $\mathbf{3}^{+}$

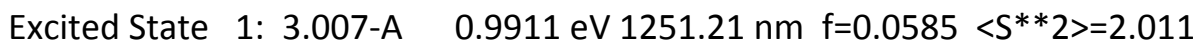
$131 \mathrm{~B}->132 \mathrm{~B} \quad 1.03707$

$131 \mathrm{~B}<-132 \mathrm{~B} \quad-0.29200$

This state for optimization and/or second-order correction.

Total Energy, E(TD-HF/TD-KS) = -3062.53988915

Copying the excited state density for this state as the 1-particle RhoCl density.

Excited State $2: \quad 3.022-\mathrm{A} \quad 1.6701 \mathrm{eV} \quad 742.36 \mathrm{~nm} \quad \mathrm{f}=0.0006<\mathrm{S}^{* *} 2>=2.033$ 131B ->133B 0.98652

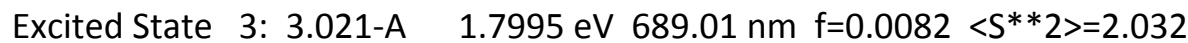
$128 \mathrm{~B}->132 \mathrm{~B} \quad 0.36490$ $130 \mathrm{~B}->132 \mathrm{~B} \quad 0.91827$

Excited State $4: 3.021-\mathrm{A} \quad 1.8391 \mathrm{eV} \quad 674.15 \mathrm{~nm} \quad \mathrm{f}=0.0015<\mathrm{S}^{* *} 2>=2.032$ $128 \mathrm{~B}->132 \mathrm{~B} \quad 0.91966$ $130 \mathrm{~B}->132 \mathrm{~B} \quad-0.36598$

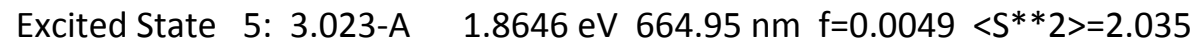
129B ->132B 0.99056

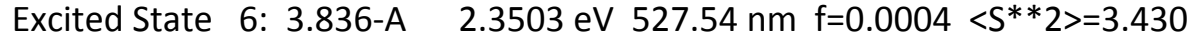
$132 \mathrm{~A} \rightarrow 134 \mathrm{~A} \quad-0.38585$

$133 \mathrm{~A} \rightarrow 135 \mathrm{~A} \quad 0.40683$

$127 \mathrm{~B}->132 \mathrm{~B} \quad 0.13391$

$131 \mathrm{~B}->134 \mathrm{~B} \quad 0.80991$

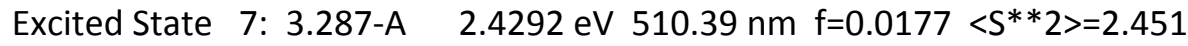
$132 \mathrm{~A} \rightarrow 135 \mathrm{~A} \quad 0.25808$

$133 \mathrm{~A} \rightarrow 134 \mathrm{~A} \quad-0.41644$

$98 \mathrm{~B}->133 \mathrm{~B} \quad-0.11160$

$99 B->133 B \quad-0.16150$

$104 B->133 B \quad-0.18417$

$109 B->133 B \quad-0.24734$

$118 \mathrm{~B}->133 \mathrm{~B} \quad 0.22654$

$120 B->133 B \quad 0.15903$

$123 B->133 B \quad 0.13076$

$126 \mathrm{~B}->132 \mathrm{~B} \quad-0.18800$

$128 \mathrm{~B}->133 \mathrm{~B} \quad 0.51646$

$130 \mathrm{~B}->133 \mathrm{~B} \quad 0.12184$

$131 B->135 B \quad-0.40178$

Excited State 8: $3.435-\mathrm{A} \quad 2.4544 \mathrm{eV} 505.15 \mathrm{~nm} \quad \mathrm{f}=0.0565<\mathrm{S} * * 2>=2.699$

$132 \mathrm{~A} \rightarrow 135 \mathrm{~A} \quad-0.26951$

$133 \mathrm{~A}->134 \mathrm{~A} \quad 0.41417$ 


$\begin{array}{cc}99 B->133 B & -0.11655 \\ 104 B->133 B & -0.14276 \\ 109 B->133 B & -0.19193 \\ 118 B->133 B & 0.16684 \\ 125 B->132 B & -0.10794 \\ 126 B->132 B & -0.36186 \\ 128 B->133 B & 0.37776 \\ 131 B->135 B & 0.54698\end{array}$

Excited State $\quad$ 9: $3.044-\mathrm{A} \quad 2.5274 \mathrm{eV} \quad 490.55 \mathrm{~nm} f=0.0036<\mathrm{S}^{* *} 2>=2.066$ 104B $->133 B \quad-0.11362$

$109 \mathrm{~B}->133 \mathrm{~B} \quad-0.13881$

$117 B->132 B \quad-0.11990$

$118 \mathrm{~B}->133 \mathrm{~B} \quad 0.12874$

$124 B->132 B \quad-0.12781$

$125 \mathrm{~B}->132 \mathrm{~B} \quad-0.12279$

$126 \mathrm{~B}->132 \mathrm{~B} \quad 0.87557$

$128 \mathrm{~B}->133 \mathrm{~B} \quad 0.23349$

$131 B->135 B \quad 0.12214$

Excited State 10: 3.070-A $2.5995 \mathrm{eV} \quad 476.95 \mathrm{~nm} f=0.0004\left\langle S^{* *} 2>=2.107\right.$

$129 \mathrm{~A}->134 \mathrm{~A} \quad-0.13151$

$130 \mathrm{~A}->135 \mathrm{~A} \quad-0.13049$

$127 \mathrm{~B}->132 \mathrm{~B} \quad 0.94177$

131B $->134 B \quad-0.19076$

Excited State 11: 3.019-A

99B $->133 B \quad-0.14830$

$102 B->133 B \quad-0.17603$

104B ->133B 0.17276

$115 B->133 B \quad 0.26887$

116B ->133B 0.21057

$118 \mathrm{~B}->133 \mathrm{~B} \quad-0.10426$

$120 \mathrm{~B}->133 \mathrm{~B} \quad 0.27438$

$123 B->133 B \quad 0.65973$

127B $->133 B \quad 0.43921$

130B $->133 B \quad-0.13649$

$131 B->135 B \quad 0.11587$

Excited State 12: $\left.3.013-\mathrm{A} \quad 2.7248 \mathrm{eV} 455.03 \mathrm{~nm} f=0.0000<S^{* *} 2\right\rangle=2.019$
$131 \mathrm{~A} \rightarrow 134 \mathrm{~A}$
$-0.12559$
$101 B->133 B$
0.20527
$105 B->133 B$
0.25441
$106 \mathrm{~B}->133 \mathrm{~B}$
0.18149
$110 \mathrm{~B}->133 \mathrm{~B}$
0.18728
$119 B->133 B \quad 0.45567$
$122 \mathrm{~B}->133 \mathrm{~B}$
0.59216
$129 B->133 B$
$-0.43909$

$2.6666 \mathrm{eV} 464.96 \mathrm{~nm} f=0.0020<S^{* *} 2>=2.029$ 
Excited State 13: 3.014-A

98B $->133 B \quad-0.10157$

$99 B->133 B \quad-0.14210$

$107 \mathrm{~B}->133 \mathrm{~B} \quad 0.14552$

$109 B->133 B \quad-0.39205$

$111 B->133 B \quad 0.19256$

$115 B->133 B \quad 0.54254$

$120 B->133 B \quad-0.28968$

$123 B->133 B \quad-0.38979$

$127 B->133 B \quad 0.24266$

$128 \mathrm{~B}->133 \mathrm{~B} \quad 0.14509$

$130 \mathrm{~B}->133 \mathrm{~B} \quad-0.30564$

Excited State 14: $3.136-\mathrm{A} \quad 2.9067 \mathrm{eV} \quad 426.54 \mathrm{~nm} \mathrm{f}=0.0985\left\langle\mathrm{~S}^{* *} 2\right\rangle=2.209$

$\begin{array}{ll}129 A->135 A & 0.15192 \\ 130 A->134 A & 0.16187 \\ 133 A->134 A & -0.18828 \\ 125 B->132 B & 0.85793 \\ 131 B->135 B & 0.33475\end{array}$

Excited State 15: $3.264-\mathrm{A} \quad 3.0415 \mathrm{eV} \quad 407.64 \mathrm{~nm} f=0.0069\left\langle\mathrm{~S}^{* *} 2>=2.413\right.$ $130 \mathrm{~A}->134 \mathrm{~A} \quad 0.13051$

$133 \mathrm{~A}->134 \mathrm{~A} \quad 0.67430$

$122 B->132 B \quad-0.11658$

$125 B->132 B \quad 0.31297$

$129 B->135 B \quad-0.10571$

$130 B->134 B \quad-0.12403$

$131 B->135 B \quad-0.57726$

Excited State 16: $3.067-\mathrm{A} \quad 3.0464 \mathrm{eV} \quad 406.99 \mathrm{~nm} f=0.1480<S^{* *} 2>=2.102$

$131 \mathrm{~A}->134 \mathrm{~A} \quad-0.14395$

$132 \mathrm{~A} \rightarrow 134 \mathrm{~A} \quad-0.49680$

$133 \mathrm{~A}->135 \mathrm{~A} \quad 0.58933$

$127 \mathrm{~B}->132 \mathrm{~B} \quad-0.12729$

$129 B->134 B \quad-0.10269$

$130 \mathrm{~B}->135 \mathrm{~B} \quad-0.11310$

$131 B->134 B \quad-0.52653$

Excited State 17: $3.221-\mathrm{A} \quad 3.2402 \mathrm{eV} 382.65 \mathrm{~nm} f=0.0014\left\langle\mathrm{~S}^{* *} 2\right\rangle=2.344$

$128 \mathrm{~A} \rightarrow 135 \mathrm{~A} \quad 0.10803$

$131 \mathrm{~A} \rightarrow 134 \mathrm{~A} \quad 0.64553$

$132 \mathrm{~A}->134 \mathrm{~A} \quad 0.50668$

$133 \mathrm{~A} \rightarrow 135 \mathrm{~A} \quad 0.50877$

Excited State 18: $3.174-\mathrm{A} \quad 3.2910 \mathrm{eV} \quad 376.73 \mathrm{~nm} f=0.0045<S^{* *} 2>=2.269$

$130 \mathrm{~A} \rightarrow 135 \mathrm{~A} \quad 0.15176$ 


$$
\begin{array}{ll}
131 \mathrm{~A}->134 \mathrm{~A} & -0.20039 \\
132 \mathrm{~A}->134 \mathrm{~A} & 0.25289 \\
120 \mathrm{~B}->132 \mathrm{~B} & 0.31037 \\
123 \mathrm{~B}->132 \mathrm{~B} & 0.81715 \\
129 \mathrm{~B}->134 \mathrm{~B} & -0.12909 \\
130 \mathrm{~B}->135 \mathrm{~B} & -0.13576
\end{array}
$$

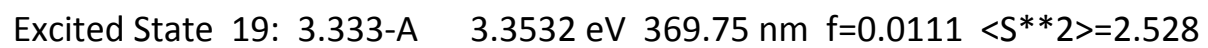

$$
\begin{array}{ll}
129 A->134 A & -0.12107 \\
130 A->135 A & -0.14476 \\
131 A->134 A & 0.64012 \\
132 A->134 A & -0.45526 \\
133 A->135 A & -0.26282 \\
120 B->132 B & 0.13009 \\
123 B->132 B & 0.40130 \\
129 B->134 B & 0.12224 \\
130 B->135 B & 0.12071
\end{array}
$$

Excited State 20: $3.234-\mathrm{A} \quad 3.3608 \mathrm{eV} 368.91 \mathrm{~nm} \mathrm{f}=0.0056\left\langle\mathrm{~S}^{* *} 2>=2.365\right.$

$$
\begin{array}{ll}
128 \mathrm{~A}->134 \mathrm{~A} & 0.11359 \\
131 \mathrm{~A}->135 \mathrm{~A} & 0.49906 \\
132 \mathrm{~A}->135 \mathrm{~A} & 0.76954 \\
133 \mathrm{~A}->134 \mathrm{~A} & 0.28306 \\
131 \mathrm{~B}->135 \mathrm{~B} & 0.16083
\end{array}
$$

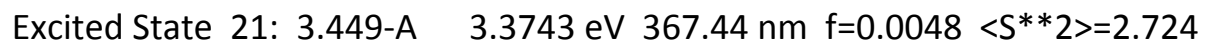

$$
\begin{array}{ll}
129 A->135 A & -0.23298 \\
130 A->134 A & -0.28283 \\
132 A->135 A & -0.11002 \\
132 A->137 A & 0.14967 \\
133 A->134 A & 0.16072 \\
133 A->136 A & -0.16865 \\
119 B->132 B & 0.32717 \\
122 B->132 B & 0.63634 \\
124 B->132 B & 0.10741 \\
125 B->132 B & 0.16555 \\
129 B->135 B & 0.22949 \\
130 B->134 B & 0.28198 \\
131 B->137 B & -0.14978
\end{array}
$$

Excited State 22: $3.846-\mathrm{A} \quad 3.4394 \mathrm{eV} \quad 360.49 \mathrm{~nm} \mathrm{f}=0.0021<\mathrm{S}^{* *} 2>=3.449$

$$
\begin{array}{ll}
129 A->134 A & 0.37287 \\
130 A->135 A & 0.35518 \\
131 A->134 A & 0.22496 \\
132 A->134 A & -0.22771 \\
132 A->136 A & -0.19083 \\
133 A->135 A & -0.35052 \\
133 A->137 A & 0.19075
\end{array}
$$




$\begin{array}{lr}123 B->132 B & -0.14798 \\ 127 B->132 B & 0.12568 \\ 129 B->134 B & -0.35418 \\ 130 B->135 B & -0.34905 \\ 131 B->136 B & 0.22575\end{array}$

Excited State 23: $3.234-\mathrm{A} \quad 3.4422 \mathrm{eV} \quad 360.19 \mathrm{~nm} f=0.0009\left\langle S^{* *} 2>=2.365\right.$

$129 \mathrm{~A} \rightarrow 135 \mathrm{~A} \quad 0.16366$

$130 \mathrm{~A} \rightarrow 134 \mathrm{~A} \quad 0.19935$

$131 \mathrm{~A} \rightarrow 135 \mathrm{~A} \quad-0.13241$

$133 A->136 A \quad 0.10157$

$122 B->132 B \quad 0.27715$

$124 \mathrm{~B}->132 \mathrm{~B} \quad 0.82065$

$125 B->132 B \quad-0.14984$

$126 B->132 B \quad 0.10239$

$129 B->135 B \quad-0.15730$

$130 \mathrm{~B}->134 \mathrm{~B} \quad-0.19440$

$131 \mathrm{~B}->137 \mathrm{~B} \quad 0.11301$

Excited State 24: $3.340-\mathrm{A} \quad 3.4553 \mathrm{eV} 358.82 \mathrm{~nm} \mathrm{f}=0.0002\left\langle\mathrm{~S}^{* *} 2\right\rangle=2.540$

$129 \mathrm{~A} \rightarrow 135 \mathrm{~A} \quad 0.18920$

$130 \mathrm{~A} \rightarrow 134 \mathrm{~A} \quad 0.23488$

$131 \mathrm{~A} \rightarrow>135 \mathrm{~A} \quad-0.28538$

$132 \mathrm{~A}->135 \mathrm{~A} \quad 0.16023$

$132 \mathrm{~A}->137 \mathrm{~A} \quad-0.11020$

$133 \mathrm{~A} \rightarrow 136 \mathrm{~A} \quad 0.12109$

$119 \mathrm{~B}->132 \mathrm{~B} \quad 0.17343$

$122 B->132 B \quad 0.53022$

$124 B->132 B \quad-0.52813$

$125 B->132 B \quad-0.15521$

$126 \mathrm{~B}->132 \mathrm{~B} \quad-0.11738$

$129 B->135 B \quad-0.18058$

$130 B->134 B \quad-0.22388$

$131 \mathrm{~B}->137 \mathrm{~B} \quad 0.13521$

Excited State 25: $3.327-\mathrm{A} \quad 3.5173 \mathrm{eV} 352.50 \mathrm{~nm} f=0.0096\left\langle\mathrm{~S}^{* *} 2>=2.517\right.$

$128 \mathrm{~A}->134 \mathrm{~A} \quad 0.13888$

$129 A->135 A \quad 0.11887$

$130 \mathrm{~A} \rightarrow 134 \mathrm{~A} \quad 0.11578$

$131 \mathrm{~A} \rightarrow 135 \mathrm{~A} \quad 0.74717$

$132 \mathrm{~A}->135 \mathrm{~A} \quad-0.44443$

$133 \mathrm{~A}->134 \mathrm{~A} \quad-0.18020$

$122 B$->132B $\quad 0.21265$

$129 B->135 B \quad-0.11283$

$130 \mathrm{~B}->133 \mathrm{~B} \quad 0.14099$

$130 \mathrm{~B}->134 \mathrm{~B} \quad-0.12417$

131B $->135 B \quad-0.12945$ 
Excited State 26: $3.015-\mathrm{A}$

121B $->132 B$

0.98804

Excited State 27: 3.036-A

$131 \mathrm{~A}->135 \mathrm{~A} \quad-0.13137$

109B $->133 B \quad-0.10446$

$111 B->133 B \quad 0.10369$

$115 B->133 B \quad 0.24834$

$127 \mathrm{~B}->133 B \quad 0.13267$

$128 \mathrm{~B}->133 \mathrm{~B} \quad-0.13565$

130B ->133B 0.89823

Excited State 28: 3.021-A

115B $->132 B \quad-0.17999$

$118 B$->132B $\quad 0.33572$

$120 B->132 B \quad 0.84653$

$123 B->132 B \quad-0.32625$

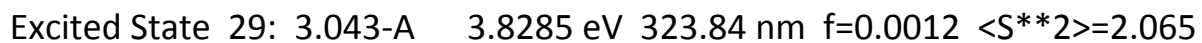
$133 \mathrm{~A} \rightarrow 137 \mathrm{~A} \quad 0.10209$

$115 B->132 B \quad-0.10282$

$116 \mathrm{~B}->132 \mathrm{~B} \quad-0.18657$

$118 \mathrm{~B}->132 \mathrm{~B} \quad 0.86055$

$120 B->132 B \quad-0.32920$

$126 B->133 B \quad 0.18612$

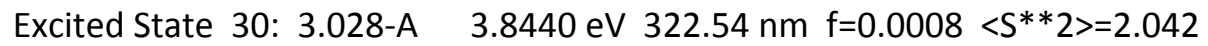
$119 \mathrm{~B}->132 \mathrm{~B} \quad 0.90716$

$122 B->132 B \quad-0.37592$

D) TD-DFT excitation energies and oscillator strengths for $4^{+}$

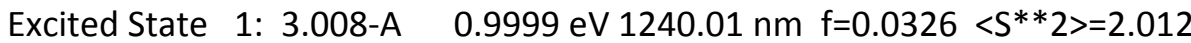
135B $->136 \mathrm{~B} \quad 1.04564$

$135 B<-136 B \quad-0.32361$

This state for optimization and/or second-order correction.

Total Energy, E(TD-HF/TD-KS) $=-3101.86460560$

Copying the excited state density for this state as the 1-particle RhoCl density.

Excited State 2: $3.018-\mathrm{A} \quad 1.4175 \mathrm{eV} 874.66 \mathrm{~nm} \mathrm{f}=0.0008<\mathrm{S}^{* *} 2>=2.027$

126B $->137 \mathrm{~B} \quad-0.10656$

$135 B->137 B \quad 0.98020$

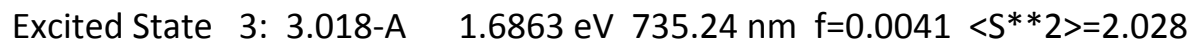

$131 \mathrm{~B}->136 \mathrm{~B} \quad 0.13000$

$132 \mathrm{~B}->136 \mathrm{~B} \quad 0.48718$

$134 \mathrm{~B}->136 \mathrm{~B} \quad 0.84922$

Excited State $4: 3.023-\mathrm{A} \quad 1.8172 \mathrm{eV} \quad 682.30 \mathrm{~nm} \quad \mathrm{f}=0.0013<\mathrm{S}^{* *} 2>=2.034$

$133 \mathrm{~B}->136 \mathrm{~B} \quad 0.99112$ 


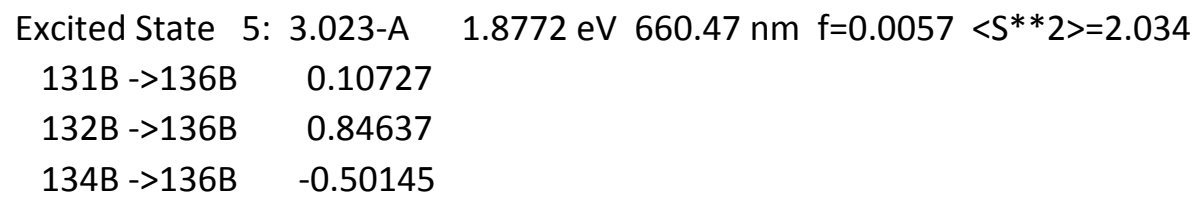

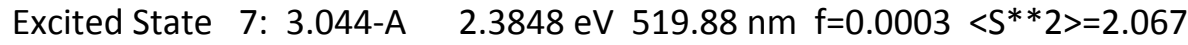
$136 \mathrm{~A} \rightarrow 139 \mathrm{~A} \quad-0.12493$

Excited State 8: $3.725-\mathrm{A} \quad 2.4112 \mathrm{eV} 514.20 \mathrm{~nm} \mathrm{f}=0.0000<\mathrm{S}^{* *} 2>=3.219$

Excited State 9: $3.753-\mathrm{A} \quad 2.4207 \mathrm{eV} 512.19 \mathrm{~nm} \mathrm{f}=0.0079<\mathrm{S}^{* *} 2>=3.272$ $136 \mathrm{~A} \rightarrow 139 \mathrm{~A} \quad-0.36836$ 


$\begin{array}{cc}\text { Excited State } 10: & 3.077-A \\ 136 \mathrm{~A}->138 \mathrm{~A} & 0.10848 \\ 137 \mathrm{~A}->139 \mathrm{~A} & 0.10068 \\ 106 \mathrm{~B}->137 \mathrm{~B} & 0.13072 \\ 108 \mathrm{~B}->137 \mathrm{~B} & 0.20924 \\ 111 \mathrm{~B}->137 \mathrm{~B} & 0.13072 \\ 118 \mathrm{~B}->137 \mathrm{~B} & -0.21479 \\ 123 \mathrm{~B}->137 \mathrm{~B} & 0.36604 \\ 126 \mathrm{~B}->137 \mathrm{~B} & 0.67014 \\ 131 \mathrm{~B}->136 \mathrm{~B} & -0.12973 \\ 133 \mathrm{~B}->137 \mathrm{~B} & -0.34894 \\ 135 \mathrm{~B}->137 \mathrm{~B} & 0.12413 \\ 135 \mathrm{~B}->138 \mathrm{~B} & -0.23358\end{array}$

Excited State 11: 3.059-A

$\begin{array}{ll}127 \mathrm{~B}->136 \mathrm{~B} & -0.12106 \\ 128 \mathrm{~B}->137 \mathrm{~B} & -0.18833 \\ 129 \mathrm{~B}->136 \mathrm{~B} & -0.32897 \\ 130 \mathrm{~B}->136 \mathrm{~B} & 0.85500 \\ 135 \mathrm{~B}->139 \mathrm{~B} & 0.17637\end{array}$

Excited State 12: 3.012-A 102B ->137B 0.19503 $104 B->137 B \quad 0.11384$ 105B $->137 B \quad 0.14156$ $109 B->137 B \quad 0.22988$ $112 B->137 B \quad 0.36954$ $116 \mathrm{~B}->137 \mathrm{~B} \quad-0.15237$ 117B $->137 B \quad-0.31010$ $121 B$->137B 0.43235 $122 B->137 B \quad 0.28081$ $128 B$->137B $\quad 0.34926$ $130 B->136 B \quad 0.14391$ $131 B->137 B \quad-0.15730$ $132 B->137 B \quad-0.26902$ $134 B$->137B 0.27103

Excited State 13: $3.088-\mathrm{A} \quad 2.5533 \mathrm{eV} \quad 485.58 \mathrm{~nm} f=0.0005\left\langle\mathrm{~S}^{* *} 2\right\rangle=2.134$ $133 \mathrm{~A} \rightarrow 138 \mathrm{~A} \quad 0.11768$ $134 \mathrm{~A} \rightarrow 139 \mathrm{~A} \quad 0.11801$ $131 \mathrm{~B}->136 \mathrm{~B} \quad 0.91797$ $132 B->136 B \quad-0.15870$ $135 B->138 B \quad-0.23958$

Excited State 14: 3.099-A $133 \mathrm{~A} \rightarrow 139 \mathrm{~A}$ $-0.14826$

$134 \mathrm{~A} \rightarrow 138 \mathrm{~A}$ $-0.14674$

$136 \mathrm{~A}->139 \mathrm{~A}$

0.12735

2.4731 eV $501.34 \mathrm{~nm} \mathrm{f}=0.0002\left\langle S^{* *} 2>=2.117\right.$

2.5256 eV $490.92 \mathrm{~nm} f=0.0060<S^{* *} 2>=2.089$

2.5467 eV $486.85 \mathrm{~nm} \mathrm{f}=0.0026\left\langle\mathrm{~S}^{* *} 2\right\rangle=2.018$

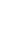




$\begin{array}{ll}137 A->138 A & 0.14466 \\ 129 B->136 B & 0.84930 \\ 130 B->136 B & 0.29782 \\ 135 B->139 B & 0.25319\end{array}$

Excited State 15: 3.116-A

134A $->138 \mathrm{~A} \quad 0.10481$

$136 \mathrm{~A} \rightarrow 139 \mathrm{~A} \quad 0.33119$

$137 \mathrm{~A}->138 \mathrm{~A} \quad 0.63946$

$126 \mathrm{~B}->136 \mathrm{~B} \quad 0.17770$

129B $->136 B \quad-0.24139$

$133 B->139 B \quad 0.11282$

$134 \mathrm{~B}->138 \mathrm{~B} \quad 0.10503$

$135 B->139 B \quad 0.54459$

Excited State 16: 3.089-A

136A $>138 \mathrm{~A} \quad 0.36127$

$137 A->139 A \quad 0.65418$

130B $->137 B \quad-0.10059$

$131 \mathrm{~B}->136 \mathrm{~B} \quad 0.10365$

135B ->138B 0.58328

Excited State 17: $3.132-\mathrm{A} \quad 3.2050 \mathrm{eV} \quad 386.84 \mathrm{~nm} \mathrm{f}=0.0005\left\langle\mathrm{~S}^{* *} 2\right\rangle=2.202$

$133 \mathrm{~A} \rightarrow 138 \mathrm{~A} \quad-0.13909$

$134 \mathrm{~A}->139 \mathrm{~A} \quad-0.14454$

$122 \mathrm{~B}->136 \mathrm{~B} \quad 0.11598$

$124 \mathrm{~B}->136 \mathrm{~B} \quad 0.18561$

$128 \mathrm{~B}->136 \mathrm{~B} \quad 0.90577$

$133 B->138 B \quad-0.12314$

$134 \mathrm{~B}->139 \mathrm{~B}-0.11436$

Excited State 18: 3.121-A $134 \mathrm{~A}->138 \mathrm{~A} \quad 0.11188$

$136 \mathrm{~A}->139 \mathrm{~A} \quad-0.40047$

$123 \mathrm{~B}->136 \mathrm{~B} \quad 0.27953$

$126 \mathrm{~B}->136 \mathrm{~B} \quad 0.79229$

$127 \mathrm{~B}->136 \mathrm{~B} \quad 0.11504$

$134 \mathrm{~B}->138 \mathrm{~B} \quad 0.10136$

$135 B->139 B \quad-0.12304$

Excited State 19: $3.270-\mathrm{A}$

$135 \mathrm{~A} \rightarrow 138 \mathrm{~A} \quad-0.29452$

$136 \mathrm{~A}->138 \mathrm{~A} \quad 0.77587$

$137 \mathrm{~A}->139 \mathrm{~A} \quad-0.51810$

Excited State $20: 3.239-\mathrm{A} \quad 3.3072 \mathrm{eV} 374.89 \mathrm{~nm} f=0.0001<S^{* *} 2>=2.373$

3.2915 eV $376.68 \mathrm{~nm} f=0.0061<S^{* *} 2>=2.185$

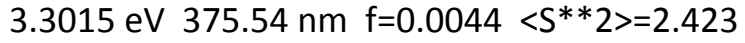

$\begin{array}{ll}135 A->139 A & -0.28044 \\ 136 A->139 A & 0.68008\end{array}$ 


$$
\begin{array}{ll}
137 \mathrm{~A}->138 \mathrm{~A} & -0.53990 \\
123 \mathrm{~B}->136 \mathrm{~B} & 0.10313 \\
126 \mathrm{~B}->136 \mathrm{~B} & 0.35531
\end{array}
$$

Excited State 21: $3.859-\mathrm{A} \quad 3.4166 \mathrm{eV} 362.89 \mathrm{~nm} \mathrm{f}=0.0057\left\langle\mathrm{~S}^{* *} 2>=3.473\right.$

$\begin{array}{lr}133 \mathrm{~A}->138 \mathrm{~A} & 0.36086 \\ 134 \mathrm{~A}->139 \mathrm{~A} & 0.36976 \\ 135 \mathrm{~A}->138 \mathrm{~A} & -0.16633 \\ 136 \mathrm{~A}->140 \mathrm{~A} & 0.20537 \\ 137 \mathrm{~A}->139 \mathrm{~A} & -0.15192 \\ 137 \mathrm{~A}->141 \mathrm{~A} & 0.21174 \\ 128 \mathrm{~B}->136 \mathrm{~B} & 0.27198 \\ 131 \mathrm{~B}->136 \mathrm{~B} & -0.14504 \\ 132 \mathrm{~B}->139 \mathrm{~B} & -0.10560 \\ 133 \mathrm{~B}->137 \mathrm{~B} & 0.24891 \\ 133 \mathrm{~B}->138 \mathrm{~B} & 0.35599 \\ 134 \mathrm{~B}->139 \mathrm{~B} & 0.36109 \\ 135 \mathrm{~B}->140 \mathrm{~B} & 0.23981\end{array}$

Excited State 22: $3.854-\mathrm{A} \quad 3.4326 \mathrm{eV} \quad 361.20 \mathrm{~nm} f=0.0024\left\langle S^{* *} 2\right\rangle=3.464$ $133 \mathrm{~A} \rightarrow 139 \mathrm{~A} \quad 0.36149$

$134 \mathrm{~A} \rightarrow 138 \mathrm{~A} \quad 0.37017$

$135 \mathrm{~A}->139 \mathrm{~A} \quad-0.17239$

$136 \mathrm{~A}->141 \mathrm{~A} \quad 0.20197$

$137 \mathrm{~A} \rightarrow>140 \mathrm{~A} \quad 0.21586$

$126 \mathrm{~B}->136 \mathrm{~B} \quad-0.26417$

$127 \mathrm{~B}->136 \mathrm{~B} \quad-0.13604$

$129 \mathrm{~B}->136 \mathrm{~B} \quad 0.22151$

$132 B->138 B \quad-0.10866$

$133 B->139 B \quad 0.35620$

$134 \mathrm{~B}->137 \mathrm{~B} \quad 0.19157$

$134 \mathrm{~B}->138 \mathrm{~B} \quad 0.35283$

$135 B->141 B \quad 0.23997$

Excited State 23: $3.028-\mathrm{A} \quad 3.4724 \mathrm{eV} \quad 357.06 \mathrm{~nm} f=0.0007<S^{* *} 2>=2.042$

$126 \mathrm{~B}->136 \mathrm{~B} \quad-0.15750$

$127 \mathrm{~B}->136 \mathrm{~B} \quad 0.96441$

$130 \mathrm{~B}->136 \mathrm{~B} \quad 0.13147$

Excited State 24: $\left.3.120-\mathrm{A} \quad 3.5496 \mathrm{eV} \quad 349.29 \mathrm{~nm} \mathrm{f}=0.0071<\mathrm{S}^{* *} 2\right\rangle=2.184$
$135 \mathrm{~A} \rightarrow 138 \mathrm{~A}$
0.41746
$136 \mathrm{~A} \rightarrow 138 \mathrm{~A}$
0.21012
$121 \mathrm{~B}->136 \mathrm{~B}$
$-0.17285$
$122 B->136 B$
$-0.19939$
$123 B->137 B$
0.10947
$124 \mathrm{~B}->136 \mathrm{~B}$
0.13882
$125 \mathrm{~B}->136 \mathrm{~B}$
$-0.16311$
$126 \mathrm{~B}->137 \mathrm{~B}$
0.19490 


$$
\begin{array}{ll}
130 \mathrm{~B}->137 \mathrm{~B} & 0.19085 \\
133 \mathrm{~B}->137 \mathrm{~B} & 0.70310
\end{array}
$$

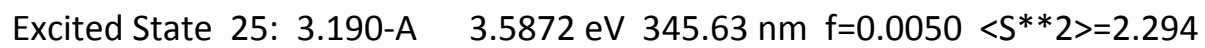

$\begin{array}{lr}132 \mathrm{~A}->138 \mathrm{~A} & 0.13238 \\ 135 \mathrm{~A}->139 \mathrm{~A} & 0.66876 \\ 136 \mathrm{~A}->139 \mathrm{~A} & 0.23747 \\ 137 \mathrm{~A}->138 \mathrm{~A} & -0.12659 \\ 117 \mathrm{~B}->137 \mathrm{~B} & 0.18344 \\ 122 \mathrm{~B}->137 \mathrm{~B} & -0.13865 \\ 131 \mathrm{~B}->137 \mathrm{~B} & 0.10715 \\ 132 \mathrm{~B}->137 \mathrm{~B} & -0.25558 \\ 134 \mathrm{~B}->137 \mathrm{~B} & 0.51827\end{array}$

Excited State 26: $3.204-\mathrm{A} \quad 3.6171 \mathrm{eV} 342.77 \mathrm{~nm} f=0.0011<S^{* *} 2>=2.317$

$\begin{array}{ll}132 \mathrm{~A}->139 \mathrm{~A} & 0.11396 \\ 133 \mathrm{~A}->138 \mathrm{~A} & 0.12874 \\ 134 \mathrm{~A}->139 \mathrm{~A} & 0.12619 \\ 135 \mathrm{~A}->138 \mathrm{~A} & 0.59692 \\ 136 \mathrm{~A}->138 \mathrm{~A} & 0.11613 \\ 137 \mathrm{~A}->139 \mathrm{~A} & -0.16579 \\ 122 \mathrm{~B}->136 \mathrm{~B} & -0.11249 \\ 125 \mathrm{~B}->136 \mathrm{~B} & -0.45598 \\ 126 \mathrm{~B}->137 \mathrm{~B} & -0.12513 \\ 133 \mathrm{~B}->137 \mathrm{~B} & -0.48066 \\ 134 \mathrm{~B}->139 \mathrm{~B} & 0.11263\end{array}$

Excited State 27: $3.096-\mathrm{A} \quad 3.6214 \mathrm{eV} \quad 342.37 \mathrm{~nm} \mathrm{f}=0.0028<S^{* *} 2>=2.146$ $135 \mathrm{~A}->138 \mathrm{~A} \quad 0.47760$

$136 \mathrm{~A} \rightarrow 138 \mathrm{~A} \quad 0.13523$

$122 \mathrm{~B}->136 \mathrm{~B} \quad 0.12713$

$125 B->136 B \quad 0.80940$

$128 \mathrm{~B}->136 \mathrm{~B}-0.13412$

Excited State 28: $3.195-\mathrm{A} \quad 3.6407 \mathrm{eV} 340.55 \mathrm{~nm} f=0.0011<S^{* *} 2>=2.302$
$132 \mathrm{~A} \rightarrow 138 \mathrm{~A}$
0.10519
$133 \mathrm{~A} \rightarrow 139 \mathrm{~A}$
0.14015
$134 \mathrm{~A} \rightarrow 138 \mathrm{~A}$
0.13668
$135 \mathrm{~A} \rightarrow>139 \mathrm{~A}$
0.57939
$136 \mathrm{~A} \rightarrow 139 \mathrm{~A}$
0.12327
$137 A->138 A \quad-0.12074$
$117 \mathrm{~B}->137 \mathrm{~B} \quad-0.15757$
$122 B->137 B \quad 0.13640$
$132 B->137 B \quad 0.31274$
$134 \mathrm{~B}->137 \mathrm{~B} \quad-0.57686$
$134 \mathrm{~B}->138 \mathrm{~B} \quad 0.12488$

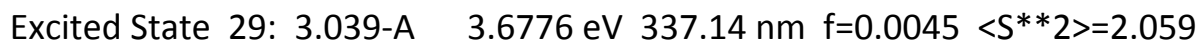




$$
\begin{array}{lr}
135 \mathrm{~A}->138 \mathrm{~A} & 0.17256 \\
117 \mathrm{~B}->136 \mathrm{~B} & -0.15274 \\
121 \mathrm{~B}->136 \mathrm{~B} & 0.16160 \\
122 \mathrm{~B}->136 \mathrm{~B} & 0.80649 \\
124 \mathrm{~B}->136 \mathrm{~B} & -0.21673 \\
125 \mathrm{~B}->136 \mathrm{~B} & -0.27615 \\
129 \mathrm{~B}->137 \mathrm{~B} & 0.11096 \\
130 \mathrm{~B}->137 \mathrm{~B} & -0.20411 \\
133 \mathrm{~B}->137 \mathrm{~B} & 0.14620
\end{array}
$$

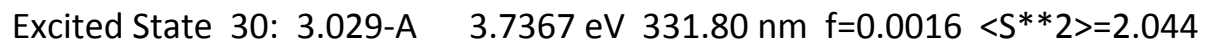

$\begin{array}{lr}135 \mathrm{~A}->138 \mathrm{~A} & -0.11400 \\ 121 \mathrm{~B}->136 \mathrm{~B} & -0.32464 \\ 122 \mathrm{~B}->136 \mathrm{~B} & 0.30757 \\ 124 \mathrm{~B}->136 \mathrm{~B} & 0.83466 \\ 128 \mathrm{~B}->136 \mathrm{~B} & -0.19440\end{array}$

E) TD-DFT excitation energies and oscillator strengths for $5^{+}$

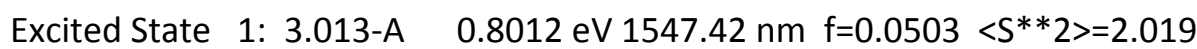
$126 \mathrm{~B}->136 \mathrm{~B} \quad-0.13217$
$130 \mathrm{~B}->136 \mathrm{~B} \quad 0.42878$
$130 \mathrm{~B}->137 \mathrm{~B} \quad-0.11049$
$131 B->136 B \quad-0.18794$
$132 \mathrm{~B}->136 \mathrm{~B} \quad-0.11133$
$133 B->136 B \quad 0.56324$
$134 \mathrm{~B}->136 \mathrm{~B} \quad 0.19402$
135B $->136 \mathrm{~B} \quad 0.59105$

This state for optimization and/or second-order correction.

Total Energy, E(TD-HF/TD-KS) $=-2881.30302442$

Copying the excited state density for this state as the 1-particle RhoCl density.

Excited State 2: $3.023-\mathrm{A} \quad 0.9564 \mathrm{eV} 1296.32 \mathrm{~nm} \quad \mathrm{f}=0.0270<\mathrm{S}^{* *} 2>=2.035$

$\begin{array}{ll}126 \mathrm{~B}->136 \mathrm{~B} & 0.31124 \\ 130 \mathrm{~B}->136 \mathrm{~B} & 0.18681 \\ 131 \mathrm{~B}->136 \mathrm{~B} & 0.67503 \\ 132 \mathrm{~B}->136 \mathrm{~B} & 0.39384 \\ 134 \mathrm{~B}->136 \mathrm{~B} & -0.24162 \\ 135 \mathrm{~B}->136 \mathrm{~B} & 0.36288\end{array}$

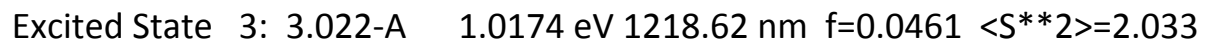

$\begin{array}{lr}130 \mathrm{~B}->137 \mathrm{~B} & 0.61518 \\ 130 \mathrm{~B}->139 \mathrm{~B} & 0.12002 \\ 133 \mathrm{~B}->136 \mathrm{~B} & -0.10112 \\ 133 \mathrm{~B}->137 \mathrm{~B} & 0.53578 \\ 135 \mathrm{~B}->136 \mathrm{~B} & 0.40402 \\ 135 \mathrm{~B}->137 \mathrm{~B} & 0.14767\end{array}$

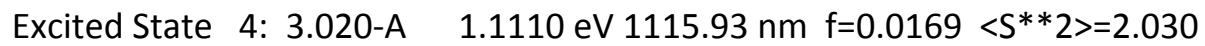
$126 \mathrm{~B}->137 \mathrm{~B} \quad 0.22666$ 


$$
\begin{array}{lr}
127 \mathrm{~B}->136 \mathrm{~B} & 0.14800 \\
130 \mathrm{~B}->136 \mathrm{~B} & -0.17553 \\
131 \mathrm{~B}->136 \mathrm{~B} & 0.11852 \\
131 \mathrm{~B}->137 \mathrm{~B} & 0.33046 \\
132 \mathrm{~B}->137 \mathrm{~B} & 0.16197 \\
133 \mathrm{~B}->136 \mathrm{~B} & -0.27365 \\
134 \mathrm{~B}->136 \mathrm{~B} & 0.74754 \\
135 \mathrm{~B}->136 \mathrm{~B} & 0.17330 \\
134 \mathrm{~B}<-136 \mathrm{~B} & 0.10225
\end{array}
$$

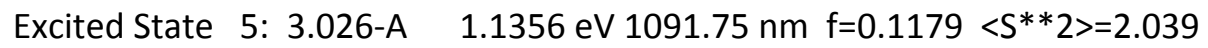
$130 \mathrm{~B}->136 \mathrm{~B} \quad-0.41547$

$130 \mathrm{~B}->137 \mathrm{~B} \quad-0.29217$

$131 B->136 B \quad-0.25419$

$132 B->136 B \quad-0.13162$

$133 B->136 B \quad-0.39395$

$133 \mathrm{~B}->137 \mathrm{~B} \quad-0.21858$

$134 \mathrm{~B}->136 \mathrm{~B} \quad-0.25431$

$135 B->136 B \quad 0.55620$

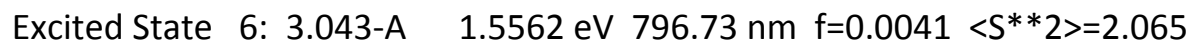

$126 \mathrm{~B}->137 \mathrm{~B} \quad 0.15210$

$127 \mathrm{~B}->137 \mathrm{~B} \quad 0.24915$

130B $>140 B \quad-0.13686$

$131 B->137 B \quad 0.40054$

$133 B->137 B \quad-0.14643$

$133 \mathrm{~B}->140 \mathrm{~B} \quad-0.11225$

$134 \mathrm{~B}->136 \mathrm{~B} \quad-0.32219$

$134 \mathrm{~B}->137 \mathrm{~B} \quad 0.70418$

$134 \mathrm{~B}->139 \mathrm{~B} \quad 0.13720$

Excited State 7: $3.030-\mathrm{A} \quad 1.8458 \mathrm{eV} \quad 671.70 \mathrm{~nm} \quad \mathrm{f}=0.0041 \quad\left\langle\mathrm{~S}^{* *} 2\right\rangle=2.045$

$126 \mathrm{~B}->137 \mathrm{~B} \quad-0.34594$

$130 B->137 B \quad-0.13866$

$131 B->136 B \quad 0.12395$

$131 \mathrm{~B}->137 \mathrm{~B} \quad-0.45751$

$132 \mathrm{~B}->136 \mathrm{~B} \quad 0.14854$

$132 B->137 B \quad-0.33756$

$134 \mathrm{~B}->136 \mathrm{~B} \quad 0.31338$

$134 \mathrm{~B}->137 \mathrm{~B} \quad 0.54737$

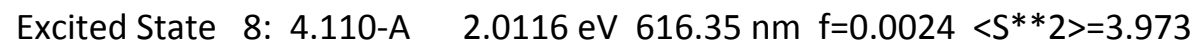

$102 \mathrm{~A}->138 \mathrm{~A} \quad 0.10602$

$124 \mathrm{~A}->138 \mathrm{~A} \quad-0.26271$

$124 \mathrm{~A}->139 \mathrm{~A} \quad-0.13793$

$126 \mathrm{~A}->138 \mathrm{~A} \quad-0.14767$

$129 \mathrm{~A}->138 \mathrm{~A} \quad 0.11746$

$133 \mathrm{~A}->138 \mathrm{~A} \quad-0.39019$ 


$$
\begin{array}{lr}
133 \mathrm{~A}->139 \mathrm{~A} & -0.19708 \\
133 \mathrm{~A}->140 \mathrm{~A} & -0.10607 \\
134 \mathrm{~A}->138 \mathrm{~A} & 0.12719 \\
135 \mathrm{~A}->138 \mathrm{~A} & 0.12417 \\
126 \mathrm{~B}->140 \mathrm{~B} & 0.34034 \\
131 \mathrm{~B}->140 \mathrm{~B} & 0.46036 \\
132 \mathrm{~B}->140 \mathrm{~B} & 0.25417 \\
133 \mathrm{~B}->140 \mathrm{~B} & -0.23762 \\
134 \mathrm{~B}->140 \mathrm{~B} & -0.10867
\end{array}
$$

Excited State 9: $3.764-\mathrm{A} \quad 2.1050 \mathrm{eV} 588.99 \mathrm{~nm} \quad \mathrm{f}=0.0001<\mathrm{S}^{* *} 2>=3.291$

$$
\begin{array}{ll}
104 \mathrm{~A}->138 \mathrm{~A} & 0.10294 \\
128 \mathrm{~A} \rightarrow 138 \mathrm{~A} & 0.10157
\end{array}
$$$$
129 \mathrm{~A}->138 \mathrm{~A} \quad 0.41634
$$$$
129 \mathrm{~A}->139 \mathrm{~A} \quad 0.21340
$$$$
129 \mathrm{~A}->140 \mathrm{~A} \quad 0.11851
$$$$
132 \mathrm{~A} \rightarrow>138 \mathrm{~A} \quad 0.16752
$$$$
135 \mathrm{~A} \rightarrow 138 \mathrm{~A} \quad 0.21050
$$$$
135 A->139 A \quad 0.10174
$$$$
136 \mathrm{~A}->138 \mathrm{~A} \quad 0.20919
$$$$
137 \mathrm{~A} \rightarrow 138 \mathrm{~A} \quad 0.42852
$$$$
137 \mathrm{~A} \rightarrow 139 \mathrm{~A} \quad 0.14278
$$$$
130 B->140 B \quad-0.37001
$$$$
131 B->140 B \quad-0.17989
$$$$
133 B->140 B \quad-0.24669
$$$$
134 \mathrm{~B}->140 \mathrm{~B} \quad-0.17517
$$

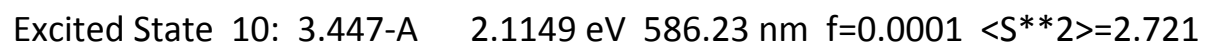
$116 \mathrm{~A} \rightarrow 138 \mathrm{~A}$
0.12482
$117 \mathrm{~A} \rightarrow 138 \mathrm{~A}$
$-0.11000$
$122 \mathrm{~A} \rightarrow 138 \mathrm{~A}$
0.10832
$129 A->138 A$
$-0.28885$
$129 A->139 A$
$-0.14738$
$132 \mathrm{~A} \rightarrow 138 \mathrm{~A}$
0.20957
$133 \mathrm{~A} \rightarrow 138 \mathrm{~A}$
$-0.12680$
$134 \mathrm{~A} \rightarrow 138 \mathrm{~A}$
$-0.17382$
$137 A->138 A$
0.61571
$137 A->139 A$
0.22248
$137 A->140 A$
0.12009
130B $->140 B$
0.26696
$133 B->140 B$
0.19157
134B ->140B
$-0.21215$

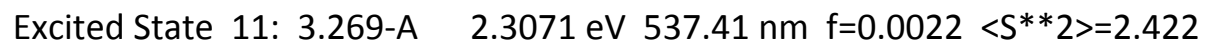
$136 \mathrm{~A} \rightarrow 138 \mathrm{~A}$
$-0.17172$
$136 \mathrm{~A}->139 \mathrm{~A}$
0.21682
$137 A->140 A$
0.31863
$131 \mathrm{~B}->136 \mathrm{~B}$
$-0.37407$ 


$\begin{array}{lr}132 \mathrm{~B}->136 \mathrm{~B} & 0.70000 \\ 134 \mathrm{~B}->139 \mathrm{~B} & -0.10414 \\ 135 \mathrm{~B}->137 \mathrm{~B} & 0.31169 \\ 135 \mathrm{~B}->138 \mathrm{~B} & 0.19527\end{array}$

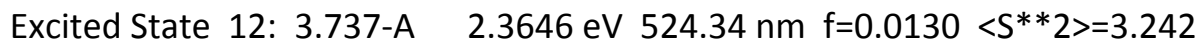

$136 \mathrm{~A} \rightarrow 138 \mathrm{~A} \quad-0.23984$

$136 \mathrm{~A} \rightarrow 140 \mathrm{~A} \quad 0.36895$

$137 \mathrm{~A}->138 \mathrm{~A} \quad-0.23026$

$137 \mathrm{~A} \rightarrow 139 \mathrm{~A} \quad 0.52340$

$130 \mathrm{~B}->136 \mathrm{~B} \quad 0.16956$

$133 B->136 B \quad-0.20170$

$134 B->138 B \quad-0.25390$

$135 B->137 B \quad-0.21562$

$135 \mathrm{~B}->138 \mathrm{~B} \quad 0.23940$

$135 B->139 B \quad 0.40460$

Excited State 13: $3.620-\mathrm{A} \quad 2.3984 \mathrm{eV} 516.94 \mathrm{~nm} \mathrm{f=0.0019}\left\langle\mathrm{S}^{* *} 2>=3.026\right.$ $136 \mathrm{~A}->138 \mathrm{~A} \quad 0.19296$

$136 \mathrm{~A}->139 \mathrm{~A} \quad-0.28116$

$137 \mathrm{~A} \rightarrow 140 \mathrm{~A} \quad-0.38050$

$131 \mathrm{~B}->136 \mathrm{~B} \quad-0.25138$

$132 B->136 B \quad 0.49527$

$134 \mathrm{~B}->139 \mathrm{~B} \quad 0.19081$

$135 B->137 B \quad-0.32947$

$135 \mathrm{~B}->138 \mathrm{~B} \quad-0.45703$

135B ->139B 0.18073

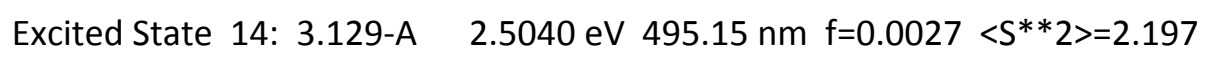
136A $>$ 139A -0.13392
$137 A->140 A \quad-0.17137$
130B $->136 \mathrm{~B} \quad 0.39541$
$133 B->136 B \quad-0.36313$
$135 B$->137B 0.69722
$135 \mathrm{~B}->138 \mathrm{~B} \quad-0.26160$
135B ->139B 0.13812

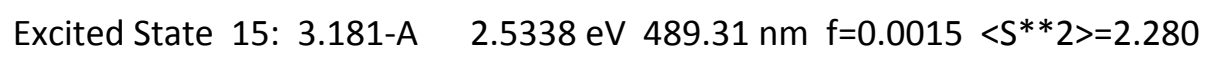

$136 \mathrm{~A}->140 \mathrm{~A} \quad-0.14958$

$137 \mathrm{~A} \rightarrow>139 \mathrm{~A} \quad-0.22508$

$130 \mathrm{~B}->136 \mathrm{~B} \quad 0.53347$

$131 \mathrm{~B}->136 \mathrm{~B} \quad-0.21400$

$133 B->136 B \quad-0.47244$

$135 B->137 B \quad-0.42916$

$135 B->139 B \quad-0.32058$

Excited State 16: $3.291-\mathrm{A} \quad 2.6359 \mathrm{eV} \quad 470.37 \mathrm{~nm} f=0.0022\left\langle\mathrm{~S}^{* *} 2>=2.457\right.$ $128 \mathrm{~A}->138 \mathrm{~A} \quad-0.15919$ 


$$
\begin{array}{ll}
131 \mathrm{~A}->138 \mathrm{~A} & -0.37317 \\
131 \mathrm{~A}->139 \mathrm{~A} & -0.18631 \\
133 \mathrm{~A}->138 \mathrm{~A} & 0.13142 \\
135 \mathrm{~A}->138 \mathrm{~A} & -0.11807 \\
136 \mathrm{~A}->138 \mathrm{~A} & 0.66307 \\
136 \mathrm{~A}->139 \mathrm{~A} & 0.30945 \\
136 \mathrm{~A}->140 \mathrm{~A} & 0.16627 \\
130 \mathrm{~B}->136 \mathrm{~B} & 0.17683 \\
135 \mathrm{~B}->138 \mathrm{~B} & 0.16593 \\
135 \mathrm{~B}->139 \mathrm{~B} & 0.11609
\end{array}
$$

Excited State 17: $3.374-\mathrm{A} \quad 2.7901 \mathrm{eV} \quad 444.37 \mathrm{~nm} f=0.0047\left\langle\mathrm{~S}^{* *} 2>=2.596\right.$

$\begin{array}{ll}124 \mathrm{~A}->138 \mathrm{~A} & 0.17742 \\ 133 \mathrm{~A}->138 \mathrm{~A} & 0.23607 \\ 137 \mathrm{~A}->138 \mathrm{~A} & -0.23551 \\ 137 \mathrm{~A}->139 \mathrm{~A} & -0.14517 \\ 120 \mathrm{~B}->136 \mathrm{~B} & -0.11994 \\ 125 \mathrm{~B}->136 \mathrm{~B} & 0.12715 \\ 126 \mathrm{~B}->140 \mathrm{~B} & 0.18170 \\ 128 \mathrm{~B}->136 \mathrm{~B} & -0.14316 \\ 129 \mathrm{~B}->136 \mathrm{~B} & 0.53560 \\ 131 \mathrm{~B}->140 \mathrm{~B} & 0.16269 \\ 132 \mathrm{~B}->140 \mathrm{~B} & 0.17643 \\ 134 \mathrm{~B}->140 \mathrm{~B} & -0.45887 \\ 135 \mathrm{~B}->138 \mathrm{~B} & -0.11751\end{array}$

Excited State 18: $3.086-\mathrm{A} \quad 2.8824 \mathrm{eV} \quad 430.15 \mathrm{~nm} f=0.0166\left\langle\mathrm{~S}^{* *} 2>=2.131\right.$ $124 \mathrm{~A}->138 \mathrm{~A} \quad-0.16455$

$133 \mathrm{~A}->138 \mathrm{~A} \quad-0.24441$

$133 \mathrm{~A} \rightarrow 139 \mathrm{~A} \quad-0.14617$

$136 \mathrm{~A} \rightarrow 138 \mathrm{~A} \quad 0.10864$

$137 A->138 A \quad-0.12578$

$137 A->139 A \quad 0.13478$

$120 B->136 B \quad-0.11538$

$125 \mathrm{~B}->136 \mathrm{~B} \quad 0.12916$

$126 B->136 B \quad-0.22974$

$126 \mathrm{~B}->140 \mathrm{~B} \quad-0.19072$

$128 \mathrm{~B}->136 \mathrm{~B} \quad-0.20670$

$129 B->136 B \quad 0.57276$

$131 \mathrm{~B}->136 \mathrm{~B} \quad 0.12378$

$131 \mathrm{~B}->138 \mathrm{~B} \quad-0.10117$

$131 B->140 B \quad-0.20686$

132B $->140 B \quad-0.15341$

$133 B->140 B \quad 0.11481$

$134 \mathrm{~B}->138 \mathrm{~B} \quad 0.18760$

134B ->140B 0.23958

135B $>139 B \quad-0.15004$ 


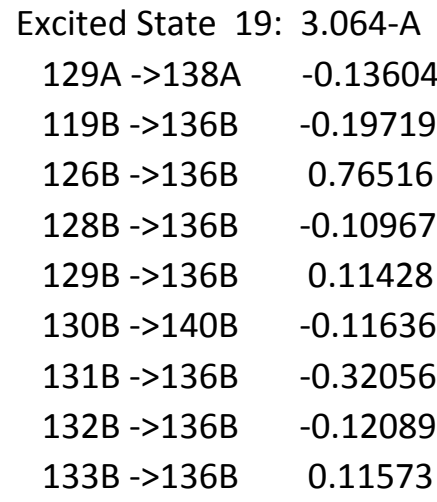

Excited State $20:$ 3.727-A $133 \mathrm{~A}->138 \mathrm{~A} \quad 0.22880$

$133 \mathrm{~A}->139 \mathrm{~A} \quad 0.10220$

136A $\rightarrow 138 \mathrm{~A} \quad-0.14800$

$137 \mathrm{~A} \rightarrow 138 \mathrm{~A} \quad 0.37955$

$126 \mathrm{~B}->140 \mathrm{~B} \quad 0.12986$

$127 \mathrm{~B}->140 \mathrm{~B} \quad 0.17972$

$129 \mathrm{~B}->136 \mathrm{~B} \quad 0.33100$

$131 B->140 B \quad 0.29527$

133B $->140 B \quad-0.14021$

$134 \mathrm{~B}->138 \mathrm{~B} \quad 0.14104$

$134 \mathrm{~B}->140 \mathrm{~B} \quad 0.50417$

$135 \mathrm{~B}->138 \mathrm{~B} \quad 0.19687$

135B ->139B 0.23855

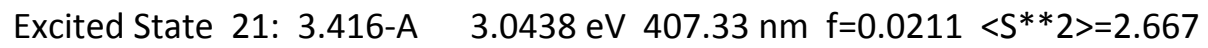

$124 \mathrm{~A}->138 \mathrm{~A} \quad 0.11148$

$131 \mathrm{~A}->138 \mathrm{~A} \quad 0.14192$

$133 \mathrm{~A}->138 \mathrm{~A} \quad 0.12160$

$136 \mathrm{~A}->138 \mathrm{~A} \quad 0.17928$

$136 \mathrm{~A}->139 \mathrm{~A} \quad 0.12141$

$137 A->138 A \quad-0.12462$

$137 \mathrm{~A}->139 \mathrm{~A} \quad 0.51194$

$137 \mathrm{~A} \rightarrow 140 \mathrm{~A} \quad 0.33235$

$126 \mathrm{~B}->140 \mathrm{~B} \quad 0.13007$

$128 \mathrm{~B}->136 \mathrm{~B} \quad 0.12176$

$131 \mathrm{~B}->140 \mathrm{~B} \quad 0.21521$

$133 B->138 B \quad-0.12655$

$134 \mathrm{~B}->138 \mathrm{~B} \quad 0.19253$

$134 \mathrm{~B}->140 \mathrm{~B} \quad 0.16306$

$135 \mathrm{~B}->138 \mathrm{~B} \quad-0.33345$

$135 B->139 B \quad-0.33994$

Excited State $\left.22: 3.361-\mathrm{A} \quad 3.0682 \mathrm{eV} \quad 404.09 \mathrm{~nm} \mathrm{f}=0.0819<\mathrm{S}^{* *} 2\right\rangle=2.574$

$131 \mathrm{~A} \rightarrow>138 \mathrm{~A} \quad-0.13305$

$135 \mathrm{~A} \rightarrow>139 \mathrm{~A} \quad-0.14270$

$136 \mathrm{~A}->138 \mathrm{~A} \quad-0.15181$

$\left.2.9446 \mathrm{eV} 421.06 \mathrm{~nm} f=0.0011<S^{* *} 2\right\rangle=2.096$

2.9563 eV $419.39 \mathrm{~nm} f=0.0072<S^{* *} 2>=3.223$

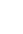




$$
\begin{array}{ll}
137 \mathrm{~A}->139 \mathrm{~A} & -0.27311 \\
137 \mathrm{~A}->140 \mathrm{~A} & 0.52312 \\
132 \mathrm{~B}->139 \mathrm{~B} & 0.14739 \\
133 \mathrm{~B}->138 \mathrm{~B} & -0.14970 \\
135 \mathrm{~B}->138 \mathrm{~B} & -0.50337 \\
135 \mathrm{~B}->139 \mathrm{~B} & 0.39266
\end{array}
$$

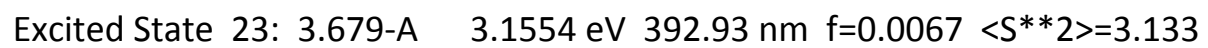

$\begin{array}{lr}128 \mathrm{~A}->138 \mathrm{~A} & 0.24468 \\ 128 \mathrm{~A}->139 \mathrm{~A} & 0.10311 \\ 131 \mathrm{~A}->138 \mathrm{~A} & 0.32078 \\ 131 \mathrm{~A}->139 \mathrm{~A} & 0.16048 \\ 133 \mathrm{~A}->138 \mathrm{~A} & -0.10033 \\ 134 \mathrm{~A}->138 \mathrm{~A} & -0.18885 \\ 134 \mathrm{~A}->139 \mathrm{~A} & 0.20823 \\ 134 \mathrm{~A}->140 \mathrm{~A} & 0.24371 \\ 136 \mathrm{~A}->138 \mathrm{~A} & 0.20338 \\ 136 \mathrm{~A}->139 \mathrm{~A} & 0.24263 \\ 136 \mathrm{~A}->140 \mathrm{~A} & 0.27490 \\ 137 \mathrm{~A}->139 \mathrm{~A} & -0.16137 \\ 127 \mathrm{~B}->136 \mathrm{~B} & 0.14572 \\ 128 \mathrm{~B}->136 \mathrm{~B} & 0.12759 \\ 132 \mathrm{~B}->138 \mathrm{~B} & -0.18412 \\ 133 \mathrm{~B}->138 \mathrm{~B} & 0.24326 \\ 133 \mathrm{~B}->139 \mathrm{~B} & 0.19185 \\ 134 \mathrm{~B}->138 \mathrm{~B} & -0.28292 \\ 134 \mathrm{~B}->140 \mathrm{~B} & 0.10525 \\ 135 \mathrm{~B}->138 \mathrm{~B} & -0.12663\end{array}$

Excited State 24: $3.439-\mathrm{A} \quad 3.1588 \mathrm{eV} 392.50 \mathrm{~nm} f=0.0138<\mathrm{S}^{* *} 2>=2.707$ $128 \mathrm{~A}->138 \mathrm{~A}$ 0.14173

$128 \mathrm{~A} \rightarrow 139 \mathrm{~A} \quad 0.10272$

$131 \mathrm{~A}->138 \mathrm{~A} \quad 0.26814$

$131 \mathrm{~A}->139 \mathrm{~A} \quad 0.12843$

$134 \mathrm{~A}->138 \mathrm{~A} \quad 0.17012$

$134 \mathrm{~A}->139 \mathrm{~A} \quad-0.12010$

$134 \mathrm{~A} \rightarrow 140 \mathrm{~A} \quad-0.20833$

$135 \mathrm{~A}->138 \mathrm{~A} \quad 0.10769$

$135 \mathrm{~A}->139 \mathrm{~A} \quad-0.15286$

$135 \mathrm{~A} \rightarrow 140 \mathrm{~A} \quad 0.16169$

136A $>138 \mathrm{~A} \quad 0.40078$

136A $>139 A \quad-0.37762$

137A $->139 A \quad-0.12905$

$137 A->140 A \quad 0.15148$

$127 \mathrm{~B}->136 \mathrm{~B} \quad-0.18235$

$132 B->139 B \quad 0.17368$

$133 B->138 B \quad-0.22831$

134B ->139B 0.16742 


$$
\begin{array}{cc}
135 B->138 B & 0.32049 \\
& \\
\hline \text { Excited State } 25: & 3.647-A \\
128 A->138 A & 0.14356 \\
131 A->138 A & 0.20908 \\
131 A->139 A & 0.11306 \\
134 A->138 A & 0.10630 \\
135 A->139 A & 0.35061 \\
135 A->140 A & -0.28487 \\
136 A->138 A & 0.12457 \\
136 A->139 A & 0.20518 \\
136 A->140 A & -0.12057 \\
137 A->139 A & -0.16360 \\
127 B->136 B & 0.15683 \\
128 B->136 B & -0.31020 \\
129 B->136 B & -0.12009 \\
132 B->138 B & 0.14233 \\
132 B->139 B & -0.17268 \\
133 B->139 B & -0.26649 \\
134 B->138 B & 0.16908 \\
134 B->139 B & -0.12146 \\
135 B->139 B & 0.35478
\end{array}
$$

Excited State 25: $3.647-\mathrm{A} \quad 3.1954 \mathrm{eV} 388.01 \mathrm{~nm} f=0.0071<S^{* *} 2>=3.076$

Excited State 26: $3.570-\mathrm{A} \quad 3.2769 \mathrm{eV} \quad 378.36 \mathrm{~nm} \quad \mathrm{f}=0.0054\left\langle\mathrm{~S}^{* *} 2\right\rangle=2.935$

$\begin{array}{ll}134 \mathrm{~A}->138 \mathrm{~A} & 0.16741 \\ 134 \mathrm{~A}->139 \mathrm{~A} & -0.16422 \\ 134 \mathrm{~A}->140 \mathrm{~A} & -0.20859 \\ 135 \mathrm{~A}->139 \mathrm{~A} & -0.12239 \\ 136 \mathrm{~A}->138 \mathrm{~A} & -0.14297 \\ 136 \mathrm{~A}->139 \mathrm{~A} & 0.55306 \\ 136 \mathrm{~A}->140 \mathrm{~A} & 0.25462 \\ 137 \mathrm{~A}->138 \mathrm{~A} & 0.14714 \\ 137 \mathrm{~A}->140 \mathrm{~A} & -0.43429 \\ 127 \mathrm{~B}->136 \mathrm{~B} & -0.23666 \\ 132 \mathrm{~B}->138 \mathrm{~B} & 0.11408 \\ 132 \mathrm{~B}->139 \mathrm{~B} & 0.14257 \\ 133 \mathrm{~B}->138 \mathrm{~B} & -0.26925 \\ 135 \mathrm{~B}->138 \mathrm{~B} & -0.11073\end{array}$

Excited State 27: $3.344-\mathrm{A} \quad 3.3245 \mathrm{eV} 372.94 \mathrm{~nm} f=0.0176<\mathrm{S}^{* *} 2>=2.545$

$135 \mathrm{~A}->139 \mathrm{~A} \quad 0.12635$

$135 \mathrm{~A} \rightarrow 140 \mathrm{~A} \quad-0.15693$

$136 \mathrm{~A} \rightarrow 139 \mathrm{~A} \quad-0.35048$

$136 \mathrm{~A} \rightarrow 140 \mathrm{~A} \quad 0.70870$

$137 \mathrm{~A} \rightarrow 138 \mathrm{~A} \quad 0.12961$

$137 \mathrm{~A} \rightarrow 139 \mathrm{~A} \quad-0.25527$

$137 A \rightarrow 140 A \quad 0.13062$

$132 \mathrm{~B}->138 \mathrm{~B} \quad 0.12408$ 


$$
\begin{array}{ll}
132 B->139 B & -0.11926 \\
133 B->139 B & -0.17633 \\
135 B->139 B & -0.30590
\end{array}
$$

Excited State 28: 3.339-A
$129 \mathrm{~A} \rightarrow 138 \mathrm{~A}$
0.26375
$129 A->139 A$
0.11596
$134 \mathrm{~A} \rightarrow 138 \mathrm{~A}$
0.13598
$135 \mathrm{~A}->138 \mathrm{~A}$
0.22007
$135 \mathrm{~A} \rightarrow 140 \mathrm{~A}$
0.10944
$136 \mathrm{~A} \rightarrow 138 \mathrm{~A}$
$-0.12504$
$136 \mathrm{~A} \rightarrow 140 \mathrm{~A}$
0.18046
$136 \mathrm{~A} \rightarrow 141 \mathrm{~A}$
$-0.10484$
$126 \mathrm{~B}->136 \mathrm{~B}$
0.16163
$127 \mathrm{~B}->136 \mathrm{~B}$
0.44092
$128 \mathrm{~B}->136 \mathrm{~B}$
0.19846
130B $->140 B$
0.27813
$133 B->140 B$
0.26485
$134 \mathrm{~B}->136 \mathrm{~B}$
$-0.10500$
$134 \mathrm{~B}->138 \mathrm{~B}$
0.32740
134B $->139 B$
0.20992
135B $->140 B$
0.27748

Excited State 29: 3.530-A 129A $>138$ A $-0.12880$

$134 \mathrm{~A}->138 \mathrm{~A}$

$-0.12556$

$136 \mathrm{~A} \rightarrow 140 \mathrm{~A}$

0.19008

$137 \mathrm{~A} \rightarrow 139 \mathrm{~A}$

$-0.11157$

$127 \mathrm{~B}->136 \mathrm{~B}$

$-0.23018$

$128 \mathrm{~B}->136 \mathrm{~B}$

0.47627

$129 B->136 B$

0.12172

130B $->140 B$

$-0.11645$

$134 \mathrm{~B}->138 \mathrm{~B}$

0.59385

$134 \mathrm{~B}->139 \mathrm{~B}$

$-0.23896$

134B $->140 B$

$-0.15134$

$135 B->139 B$

0.21678

$135 B->140 B$

$-0.17004$

Excited State 30: 3.364-A

$129 \mathrm{~A}->138 \mathrm{~A}$

$-0.19993$

$129 \mathrm{~A} \rightarrow>139 \mathrm{~A}$

$-0.11844$

$134 \mathrm{~A}->138 \mathrm{~A}$

$-0.17666$

$135 \mathrm{~A}->138 \mathrm{~A}$

$-0.13060$

$135 \mathrm{~A}->139 \mathrm{~A}$

$-0.14325$

$136 \mathrm{~A} \rightarrow 140 \mathrm{~A}$

0.11172

$137 \mathrm{~A}->142 \mathrm{~A}$

$-0.10258$

$126 \mathrm{~B}->136 \mathrm{~B}$

$-0.18072$

$127 \mathrm{~B}->136 \mathrm{~B}$

0.39765

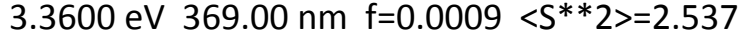

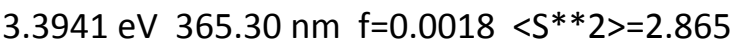




$$
\begin{array}{ll}
128 \mathrm{~B}->136 \mathrm{~B} & -0.31057 \\
129 \mathrm{~B}->136 \mathrm{~B} & -0.19587 \\
130 \mathrm{~B}->140 \mathrm{~B} & -0.23994 \\
133 \mathrm{~B}->140 \mathrm{~B} & -0.22710 \\
134 \mathrm{~B}->138 \mathrm{~B} & 0.30183 \\
134 \mathrm{~B}->139 \mathrm{~B} & 0.33729 \\
135 \mathrm{~B}->138 \mathrm{~B} & 0.11537 \\
135 \mathrm{~B}->140 \mathrm{~B} & -0.26916
\end{array}
$$

F) TD-DFT excitation energies and oscillator strengths for $\mathbf{6}^{+}$

$\begin{array}{ccc}\text { Excited State } 1: & 2.020-\mathrm{A} & 0.2974 \mathrm{eV} 4169.31 \mathrm{~nm} \mathrm{f}=0.0089<\mathrm{S}^{* *} 2>=0.770 \\ 150 \mathrm{~B} \rightarrow 151 \mathrm{~B} & 0.99779\end{array}$ This state for optimization and/or second-order correction.

Total Energy, E(TD-HF/TD-DFT) = -3072.73098037

Copying the excited state density for this state as the 1-particle RhoCl density.

Excited State $2: \quad 3.454-\mathrm{A} \quad 1.0278$ eV $1206.29 \mathrm{~nm} \quad \mathrm{f}=0.0000<\mathrm{S}^{* *} 2>=2.733$
$138 \mathrm{~A}->153 \mathrm{~A} \quad-0.11117$
$140 \mathrm{~A} \rightarrow 153 \mathrm{~A} \quad 0.12213$
$142 \mathrm{~A} \rightarrow 153 \mathrm{~A} \quad 0.11090$
$143 A->152 A \quad 0.14839$
$143 \mathrm{~A} \rightarrow 153 \mathrm{~A} \quad 0.20964$
$147 \mathrm{~A} \rightarrow 152 \mathrm{~A} \quad 0.17998$
$147 \mathrm{~A} \rightarrow 153 \mathrm{~A} \quad 0.24671$
$148 \mathrm{~A} \rightarrow 152 \mathrm{~A} \quad 0.12007$
$148 \mathrm{~A} \rightarrow 153 \mathrm{~A} \quad 0.20253$
$151 \mathrm{~A} \rightarrow 152 \mathrm{~A} \quad-0.24852$
$151 \mathrm{~A}->153 \mathrm{~A} \quad-0.35757$
$138 \mathrm{~B}->152 \mathrm{~B} \quad 0.10430$
$140 \mathrm{~B}->152 \mathrm{~B} \quad-0.21325$
$141 B->152 B \quad-0.12197$
$142 B->152 B \quad-0.15394$
$143 B->152 B \quad 0.16193$
$147 \mathrm{~B}->152 B \quad-0.34627$
$147 \mathrm{~B}->153 B \quad-0.11461$
$150 \mathrm{~B}->152 \mathrm{~B} \quad 0.38881$
$150 \mathrm{~B}->153 \mathrm{~B} \quad 0.13923$

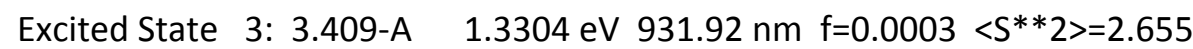

139A $->153 \mathrm{~A} \quad-0.11011$

$140 A->154 A \quad-0.10293$

$143 A->152 A \quad 0.11915$

$143 \mathrm{~A} \rightarrow 153 \mathrm{~A} \quad 0.14417$

144A $->152 A \quad-0.15832$

$144 \mathrm{~A}->153 \mathrm{~A} \quad-0.28730$

$144 \mathrm{~A}->154 \mathrm{~A} \quad-0.10169$

$147 A->152 A \quad 0.14103$

$147 A->153 A \quad 0.15521$

$148 \mathrm{~A} \rightarrow 153 \mathrm{~A} \quad-0.21675$

$148 \mathrm{~A} \rightarrow 154 \mathrm{~A} \quad-0.17225$ 


$$
\begin{array}{lr}
150 \mathrm{~A}->152 \mathrm{~A} & -0.12264 \\
150 \mathrm{~A}->153 \mathrm{~A} & -0.19200 \\
151 \mathrm{~A}->154 \mathrm{~A} & 0.20025 \\
133 \mathrm{~B}->152 \mathrm{~B} & 0.13792 \\
139 \mathrm{~B}->152 \mathrm{~B} & 0.11551 \\
139 \mathrm{~B}->153 \mathrm{~B} & 0.10015 \\
140 \mathrm{~B}->154 \mathrm{~B} & 0.10521 \\
142 \mathrm{~B}->152 \mathrm{~B} & -0.12794 \\
144 \mathrm{~B}->152 \mathrm{~B} & 0.28029 \\
144 \mathrm{~B}->153 \mathrm{~B} & 0.15166 \\
146 \mathrm{~B}->152 \mathrm{~B} & 0.10688 \\
147 \mathrm{~B}->153 \mathrm{~B} & 0.11693 \\
147 \mathrm{~B}->154 \mathrm{~B} & 0.13544 \\
148 \mathrm{~B}->152 \mathrm{~B} & 0.30175 \\
148 \mathrm{~B}->153 \mathrm{~B} & 0.16049 \\
150 \mathrm{~B}->153 \mathrm{~B} & -0.15942 \\
150 \mathrm{~B}->154 \mathrm{~B} & -0.15184
\end{array}
$$

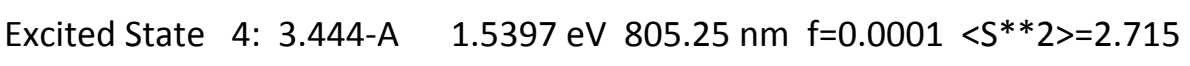
140A $>154 \mathrm{~A}$
$-0.13134$
$141 A->154 A$
$-0.15851$
$143 \mathrm{~A}->153 \mathrm{~A}$
$-0.14103$
$144 A->152 A$
0.12591
$144 \mathrm{~A} \rightarrow 153 \mathrm{~A}$
0.14435
$145 \mathrm{~A} \rightarrow 154 \mathrm{~A}$
0.10923
$147 \mathrm{~A} \rightarrow 153 \mathrm{~A}$
$-0.11599$
$147 A->154 A$
$-0.18098$
$148 \mathrm{~A} \rightarrow 152 \mathrm{~A}$
0.12373
$148 \mathrm{~A} \rightarrow 154 \mathrm{~A}$
$-0.18452$
149A $>>154 A$
0.10643
$151 \mathrm{~A}->152 \mathrm{~A}$
$-0.10472$
$151 \mathrm{~A} \rightarrow 154 \mathrm{~A}$
0.34484
$139 B->152 B$
$-0.12530$
$140 B->152 B$
$-0.12612$
140B $->153 B$
0.10081
$140 B->154 B$
0.17381
$142 B->152 B$
0.14626
$144 B->152 B$
$-0.15168$
$145 B->152 B$
$-0.16855$
$145 B$->154B
0.10156
$147 \mathrm{~B}->153 \mathrm{~B}$
0.14722
$147 \mathrm{~B}->154 \mathrm{~B}$
0.19729
$148 \mathrm{~B}->152 \mathrm{~B}$
$-0.18167$
$150 B->152 B$
0.14652
$150 \mathrm{~B}->153 \mathrm{~B}$
$-0.20644$
$150 B$->154B
$-0.26368$

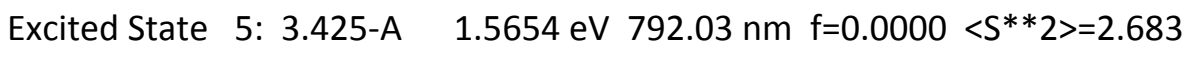

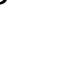




$$
\begin{array}{lr}
145 \mathrm{~A}->153 \mathrm{~A} & 0.27286 \\
145 \mathrm{~A}->154 \mathrm{~A} & -0.27338 \\
148 \mathrm{~A}->154 \mathrm{~A} & -0.15508 \\
149 \mathrm{~A}->153 \mathrm{~A} & 0.10388 \\
150 \mathrm{~A}->154 \mathrm{~A} & -0.11540 \\
131 \mathrm{~B}->152 \mathrm{~B} & -0.11515 \\
142 \mathrm{~B}->152 \mathrm{~B} & -0.19291 \\
143 \mathrm{~B}->152 \mathrm{~B} & 0.12913 \\
144 \mathrm{~B}->152 \mathrm{~B} & -0.16780 \\
144 \mathrm{~B}->154 \mathrm{~B} & 0.14744 \\
145 \mathrm{~B}->152 \mathrm{~B} & 0.36385 \\
145 \mathrm{~B}->154 \mathrm{~B} & -0.19290 \\
148 \mathrm{~B}->152 \mathrm{~B} & -0.11872 \\
148 \mathrm{~B}->154 \mathrm{~B} & 0.11606 \\
149 \mathrm{~B}->152 \mathrm{~B} & -0.13496 \\
150 \mathrm{~B}->154 \mathrm{~B} & -0.10780
\end{array}
$$

Excited State 6: $2.053-\mathrm{A} \quad 1.7298 \mathrm{eV} 716.76 \mathrm{~nm} f=0.0031<S^{* *} 2>=0.804$
$143 \mathrm{~A}->153 \mathrm{~A}$
$-0.11901$
$147 \mathrm{~A} \rightarrow 152 \mathrm{~A}$
$-0.10936$
$147 \mathrm{~A} \rightarrow 153 \mathrm{~A}$
$-0.15081$
$148 \mathrm{~A} \rightarrow 153 \mathrm{~A}$
$-0.16042$
$151 A->152 A$
0.34426
$151 \mathrm{~A} \rightarrow 153 \mathrm{~A} \quad 0.44539$
$140 \mathrm{~B}->152 \mathrm{~B} \quad-0.12806$
$142 B->152 B \quad-0.11415$
$147 \mathrm{~B}->152 \mathrm{~B} \quad-0.24816$
$147 \mathrm{~B}->153 \mathrm{~B} \quad-0.10018$
$149 \mathrm{~B}->151 \mathrm{~B} \quad 0.11969$
$150 B->152 B \quad 0.53443$
$150 B->153 B \quad 0.19526$

Excited State 7: 2.252-A $145 \mathrm{~A}->154 \mathrm{~A} \quad-0.10978$

$145 B \rightarrow 151 B \quad-0.12166$

$148 \mathrm{~B}->151 \mathrm{~B} \quad 0.12142$

$149 \mathrm{~B} \rightarrow 151 \mathrm{~B} \quad 0.90448$

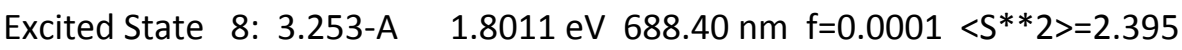
$132 \mathrm{~A} \rightarrow 154 \mathrm{~A}$
$-0.11553$
$133 A->154 A$
0.10226
$135 \mathrm{~A}->154 \mathrm{~A}$
$-0.10650$
$143 \mathrm{~A}->154 \mathrm{~A}$
$-0.15094$
$144 \mathrm{~A} \rightarrow 154 \mathrm{~A}$
0.26535
$145 \mathrm{~A} \rightarrow 153 \mathrm{~A}$
0.20685
$145 \mathrm{~A} \rightarrow 154 \mathrm{~A}$
0.14893
$147 \mathrm{~A} \rightarrow 154 \mathrm{~A}$
$-0.22066$
$148 \mathrm{~A}->154 \mathrm{~A}$
0.14336
$150 \mathrm{~A}->154 \mathrm{~A}$
0.22220
$151 \mathrm{~A}->153 \mathrm{~A}$
0.14239
$151 \mathrm{~A}->154 \mathrm{~A}$
0.16174
$133 B$->154B
$-0.11324$

1.7537 eV $706.99 \mathrm{~nm} f=0.0009\left\langle S^{* *} 2\right\rangle=1.018$

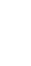




$$
\begin{array}{lr}
142 B->152 B & -0.12977 \\
144 B->153 B & -0.17969 \\
144 B->154 B & -0.21100 \\
145 B->152 B & 0.18479 \\
145 B->153 B & 0.11174 \\
148 B->153 B & -0.17550 \\
148 B->154 B & -0.21323 \\
149 B->151 B & 0.33377 \\
150 B->152 B & -0.12060
\end{array}
$$

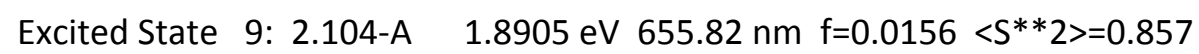
$\begin{array}{ll}146 B->151 B & -0.50162 \\ 147 B->151 B & -0.55890 \\ 148 B->151 B & 0.59823 \\ 149 B->151 B & -0.12776\end{array}$

Excited State 10: $3.407-\mathrm{A} \quad 1.9372 \mathrm{eV} 640.01 \mathrm{~nm} \mathrm{f}=0.0001<\mathrm{S}^{* *} 2>=2.652$
$131 \mathrm{~A} \rightarrow 154 \mathrm{~A} \quad-0.15766$
$139 \mathrm{~A} \rightarrow 154 \mathrm{~A} \quad 0.10812$
$141 \mathrm{~A} \rightarrow 154 \mathrm{~A} \quad 0.16665$
$143 \mathrm{~A} \rightarrow 154 \mathrm{~A} \quad-0.26524$
$144 \mathrm{~A} \rightarrow 154 \mathrm{~A} \quad 0.14702$
$145 \mathrm{~A} \rightarrow 153 \mathrm{~A} \quad-0.21736$
$145 \mathrm{~A} \rightarrow 154 \mathrm{~A} \quad-0.35547$
$147 \mathrm{~A} \rightarrow 154 \mathrm{~A} \quad-0.14864$
$149 \mathrm{~A}->154 \mathrm{~A} \quad-0.14174$
$151 \mathrm{~A} \rightarrow 154 \mathrm{~A} \quad 0.11458$
$142 B->153 B \quad 0.21540$
$142 B->154 B \quad 0.23242$
$145 B->152 B \quad-0.10377$
$145 B->153 B \quad-0.29073$
$145 B->154 B \quad-0.28249$
$148 \mathrm{~B} \rightarrow 154 \mathrm{~B} \quad-0.11067$
$149 B->151 B \quad-0.12630$
$149 \mathrm{~B} \rightarrow 153 \mathrm{~B} \quad 0.12202$
$149 \mathrm{~B}->154 \mathrm{~B} \quad 0.11372$

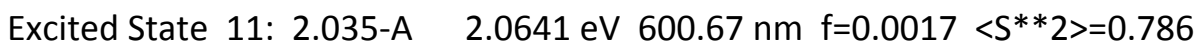
$140 \mathrm{~A}->154 \mathrm{~A} \quad-0.10506$

$144 \mathrm{~A}->153 \mathrm{~A} \quad-0.13231$

$144 \mathrm{~A}->154 \mathrm{~A} \quad-0.13095$

$148 \mathrm{~A}->153 \mathrm{~A} \quad-0.11270$

$148 \mathrm{~A}->154 \mathrm{~A} \quad-0.18921$

$150 A->153 A \quad-0.10251$

$151 A->152 A \quad-0.23552$

$151 \mathrm{~A} \rightarrow 153 \mathrm{~A} \quad 0.11983$

$151 \mathrm{~A} \rightarrow 154 \mathrm{~A} \quad 0.45651$

$140 B->154 B \quad-0.10052$

$144 B->152 B \quad-0.14050$

147B $->153 B \quad-0.11396$

147B $->154 B \quad-0.14975$

148B $->151 B \quad-0.15523$ 


$$
\begin{array}{lr}
148 B->152 B & -0.14079 \\
150 B->153 B & 0.39263 \\
150 B->154 B & 0.36483
\end{array}
$$

Excited State 12: 3.449-A $151 \mathrm{~A}->153 \mathrm{~A} \quad-0.25581$ $151 A->155 A \quad-0.62979$ $150 B->152 B \quad 0.14152$ 150B $>153 B \quad 0.19535$ 150B $->155 B \quad 0.63286$

Excited State 13: 2.048-A 151A $->154 \mathrm{~A} \quad 0.10883$ $143 B->151 B \quad 0.10345$ 145B $->151 B \quad-0.36749$ $146 \mathrm{~B} \rightarrow 151 \mathrm{~B} \quad 0.35803$ $147 \mathrm{~B}->151 \mathrm{~B} \quad 0.42518$ $148 \mathrm{~B}->151 \mathrm{~B} \quad 0.66449$ $150 \mathrm{~B}->153 \mathrm{~B} \quad 0.10134$

Excited State 14: 2.637-A $\begin{array}{ll}143 A->153 A & 0.13951 \\ 147 A->153 A & 0.14157 \\ 151 A->152 A & 0.72374 \\ 151 A->153 A & -0.26212 \\ 144 B->151 B & -0.17248 \\ 145 B->151 B & -0.30685 \\ 148 B->152 B & -0.12196 \\ 150 B->152 B & -0.11221\end{array}$

Excited State 15: 2.338-A 151A $\rightarrow 152 A \quad 0.39299$ $151 \mathrm{~A} \rightarrow 153 \mathrm{~A} \quad-0.16639$ $151 \mathrm{~A} \rightarrow 154 \mathrm{~A} \quad 0.19513$ $143 B->151 B \quad-0.15724$ $144 \mathrm{~B}->151 \mathrm{~B} \quad 0.28456$ $145 B->151 B \quad 0.62891$ 147B $->151 B \quad 0.25571$ $148 B->151 B \quad 0.26593$ $150 B->152 B \quad-0.11469$ $150 \mathrm{~B}->153 \mathrm{~B} \quad 0.10545$

Excited State 16: 2.490-A 144A $\rightarrow 153 \mathrm{~A}$ $-0.15159$ $145 \mathrm{~A} \rightarrow 154 \mathrm{~A} \quad-0.11896$ $148 \mathrm{~A} \rightarrow 153 \mathrm{~A} \quad-0.13221$ $150 A-152 A \quad-0.10424$ $150 \mathrm{~A} \rightarrow 153 \mathrm{~A} \quad-0.10198$ $151 \mathrm{~A} \rightarrow 152 \mathrm{~A} \quad-0.12080$ $151 \mathrm{~A}->153 \mathrm{~A} \quad-0.10511$ $151 \mathrm{~A}->154 \mathrm{~A} \quad-0.15645$ $142 B->152 B \quad 0.17052$
2.1261 eV $583.15 \mathrm{~nm} f=0.0000<S^{* *} 2>=2.723$

2.1306 eV $581.93 \mathrm{~nm} \mathrm{f}=0.0179<S^{* *} 2>=0.799$

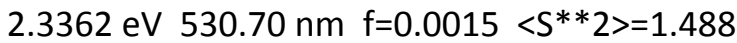

2.3735 eV $522.37 \mathrm{~nm} \mathrm{f}=0.0055<\mathrm{S}^{* *} 2>=1.116$ 


$$
\begin{array}{lr}
143 B->151 B & -0.13932 \\
143 B->152 B & -0.12394 \\
144 B->152 B & -0.12258 \\
144 B->153 B & -0.11534 \\
145 B->151 B & 0.14510 \\
145 B->152 B & -0.10383 \\
146 B->151 B & -0.31163 \\
147 B->151 B & 0.34236 \\
147 B->152 B & 0.17311 \\
148 B->151 B & 0.11368 \\
148 B->152 B & -0.13977 \\
148 B->153 B & -0.10745 \\
150 B->152 B & 0.41453 \\
150 B->153 B & -0.10258 \\
150 B->155 B & -0.10418
\end{array}
$$

Excited State 17: $2.604-\mathrm{A}$ 141A $\rightarrow 154 A \quad 0.10078$ $143 A->152 A \quad 0.10469$ $143 \mathrm{~A}->153 \mathrm{~A} \quad 0.13807$ $145 \mathrm{~A}->152 \mathrm{~A} \quad 0.10817$ $145 \mathrm{~A} \rightarrow 154 \mathrm{~A} \quad-0.21573$ $150 \mathrm{~A} \rightarrow 154 \mathrm{~A} \quad-0.10425$ $151 \mathrm{~A} \rightarrow 153 \mathrm{~A} \quad 0.38175$ $151 \mathrm{~A}->154 \mathrm{~A} \quad-0.16153$ $151 A->155 A \quad-0.17444$ $139 B->152 B \quad-0.10408$ $140 B->152 B \quad-0.14475$ $142 B->152 B \quad 0.10659$ 144B $->151 B \quad 0.12491$ $144 B->153 B \quad-0.10233$ $144 B->154 B \quad-0.10510$ 145B $->151 B \quad 0.26713$ 145B $->152 B \quad-0.19976$ $145 B->154 B \quad 0.14891$ $146 B->151 B \quad 0.29475$ $147 \mathrm{~B} \rightarrow 151 \mathrm{~B} \quad-0.14924$ $147 \mathrm{~B} \rightarrow 152 B \quad-0.10069$ $148 \mathrm{~B}->151 \mathrm{~B} \quad 0.13765$ $148 \mathrm{~B} \rightarrow 153 \mathrm{~B} \quad-0.11620$ $149 B->152 B \quad 0.12609$ 150B $->152 B \quad-0.23118$ $150 B->153 B \quad-0.11392$ 150B $->155 B \quad 0.15160$

Excited State 18: $2.511-\mathrm{A} \quad 2.4752 \mathrm{eV} 500.90 \mathrm{~nm} \quad \mathrm{f}=0.0188<\mathrm{S}^{* *} 2>=1.327$
2.4600 eV 503.99 nm f $=0.0122\langle S * * 2\rangle=1.445$

8
$151 \mathrm{~A} \rightarrow 153 \mathrm{~A} \quad-0.32839$
$140 \mathrm{~B}->152 \mathrm{~B} \quad 0.10222$
$143 B->151 B \quad 0.14751$
$144 \mathrm{~B}->151 \mathrm{~B} \quad 0.17007$
145B $->151 B \quad 0.13890$ 


$$
\begin{array}{cc}
146 B->151 B & 0.52832 \\
147 B->151 B & -0.44632 \\
147 B->152 B & 0.11795 \\
150 B->152 B & 0.30635 \\
150 B->153 B & -0.18653 \\
150 B->155 B & -0.12829
\end{array}
$$

Excited State 19: 2.437-A$$
\text { 141A }>154 \mathrm{~A} \quad 0.12304
$$$$
143 \mathrm{~A} \rightarrow 153 \mathrm{~A} \quad-0.10109
$$$$
145 \mathrm{~A} \rightarrow 152 \mathrm{~A} \quad 0.16092
$$$$
145 \mathrm{~A} \rightarrow 153 \mathrm{~A} \quad 0.10085
$$$$
145 \mathrm{~A} \rightarrow 154 \mathrm{~A} \quad-0.26314
$$$$
147 \mathrm{~A} \rightarrow 153 \mathrm{~A} \quad-0.14184
$$$$
149 \mathrm{~A} \rightarrow 154 \mathrm{~A} \quad-0.10617
$$$$
150 \mathrm{~A} \rightarrow 152 \mathrm{~A} \quad 0.14670
$$$$
150 \mathrm{~A} \rightarrow 153 \mathrm{~A} \quad 0.17197
$$$$
151 \mathrm{~A} \rightarrow 153 \mathrm{~A} \quad-0.26361
$$$$
151 \mathrm{~A} \rightarrow 154 \mathrm{~A} \quad 0.20322
$$$$
151 \mathrm{~A} \rightarrow 155 \mathrm{~A} \quad 0.14338
$$$$
\text { 140B }->154 B \quad 0.10609
$$$$
\text { 144B }->151 B \quad 0.12636
$$$$
144 B->152 B \quad 0.20272
$$$$
\text { 145B }->152 B \quad-0.16112
$$$$
\text { 145B }->153 B \quad 0.13318
$$$$
145 B->154 B \quad 0.20281
$$$$
146 \mathrm{~B} \rightarrow 152 \mathrm{~B} \quad 0.10097
$$$$
148 \mathrm{~B}->152 \mathrm{~B} \quad 0.21018
$$$$
149 B->154 B \quad-0.10208
$$$$
150 B->152 B \quad-0.10616
$$$$
\text { 150B }->153 B \quad 0.39929
$$$$
\text { 150B }->155 B \quad-0.10398
$$

Excited State 20: 2.642-A
$150 \mathrm{~A}->152 \mathrm{~A}$
0.23705
$151 \mathrm{~A}->154 \mathrm{~A}$
0.24869
$144 \mathrm{~B}->152 \mathrm{~B}$
0.11658
$146 B->151 B$
$-0.15859$
$148 \mathrm{~B}->152 \mathrm{~B}$
0.10414
$150 \mathrm{~B}->152 \mathrm{~B}$
0.10209
$150 \mathrm{~B}->153 \mathrm{~B}$
$-0.57030$
$150 \mathrm{~B}->154 \mathrm{~B}$
0.59791
$150 B->155 B$
0.14130

Excited State 21: 2.039-A
$143 \mathrm{~A} \rightarrow 152 \mathrm{~A}$
0.12504
$143 \mathrm{~A} \rightarrow 153 \mathrm{~A}$
0.15632
$144 \mathrm{~A} \rightarrow 154 \mathrm{~A}$
0.15748
$145 \mathrm{~A} \rightarrow 153 \mathrm{~A}$
0.12873
$147 A->152 A$
0.13356
$147 A->153 A$
0.12483
$148 \mathrm{~A} \rightarrow 153 \mathrm{~A}$
0.16265

2.6180 eV $473.59 \mathrm{~nm} f=0.0008\left\langle S^{* *} 2>=1.495\right.$

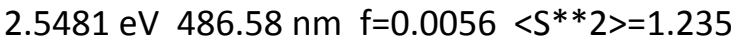




$$
\begin{array}{ll}
148 \mathrm{~A}->154 \mathrm{~A} & 0.12836 \\
150 \mathrm{~A}->154 \mathrm{~A} & 0.16108 \\
151 \mathrm{~A}->152 \mathrm{~A} & 0.14415 \\
151 \mathrm{~A}->153 \mathrm{~A} & 0.30002 \\
151 \mathrm{~A}->154 \mathrm{~A} & 0.18345 \\
140 \mathrm{~B}->152 \mathrm{~B} & 0.11937 \\
142 \mathrm{~B}->152 \mathrm{~B} & 0.14304 \\
143 \mathrm{~B}->152 \mathrm{~B} & -0.15798 \\
144 \mathrm{~B}->151 \mathrm{~B} & 0.20158 \\
144 \mathrm{~B}->153 \mathrm{~B} & 0.14840 \\
144 \mathrm{~B}->154 \mathrm{~B} & 0.14962 \\
145 \mathrm{~B}->151 \mathrm{~B} & -0.10491 \\
145 \mathrm{~B}->152 \mathrm{~B} & -0.12480 \\
147 \mathrm{~B}->151 \mathrm{~B} & -0.10471 \\
147 \mathrm{~B}->152 \mathrm{~B} & 0.23375 \\
148 \mathrm{~B}->153 \mathrm{~B} & 0.14066 \\
148 \mathrm{~B}->154 \mathrm{~B} & 0.16330 \\
150 \mathrm{~B}->152 \mathrm{~B} & 0.30142 \\
150 \mathrm{~B}->153 \mathrm{~B} & 0.16880
\end{array}
$$

Excited State 22: 2.109-A

$$
\begin{array}{lr}
150 A->152 A & 0.14663 \\
150 A->153 A & -0.12678 \\
151 A->154 A & -0.16550 \\
142 B->151 B & 0.19807 \\
143 B->151 B & 0.26612 \\
144 B->151 B & 0.74654 \\
145 B->151 B & -0.30209 \\
146 B->151 B & -0.19345
\end{array}
$$

Excited State 23: 3.429-A 141A $->154 \mathrm{~A} \quad-0.12083$ $142 A->154 A \quad-0.10621$ $143 \mathrm{~A}->154 \mathrm{~A} \quad-0.18035$ $147 A->154 A \quad-0.18664$ $148 \mathrm{~A}->154 \mathrm{~A} \quad-0.15153$ $151 \mathrm{~A}->154 \mathrm{~A} \quad-0.49607$ $140 \mathrm{~B}->153 \mathrm{~B} \quad 0.12425$ $140 B->154 B \quad 0.14592$ $143 B->153 B \quad-0.10259$ $143 B->154 B \quad-0.12128$ $147 \mathrm{~B}->153 \mathrm{~B} \quad 0.18123$ $147 \mathrm{~B}->154 \mathrm{~B} \quad 0.18935$ $150 \mathrm{~B}->153 \mathrm{~B} \quad 0.15174$ 150B $->154 B \quad 0.54695$

Excited State 24: 2.053-A $\begin{array}{ll}150 A->152 A & 0.29525 \\ 150 A->153 A & -0.16903 \\ 140 B->151 B & 0.11351 \\ 143 B->151 B & 0.74890 \\ 144 B->151 B & -0.31209\end{array}$

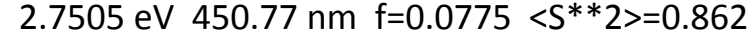

2.7924 eV $444.00 \mathrm{~nm} \mathrm{f}=0.0017<S^{* *} 2>=2.690$

2.8579 eV $433.84 \mathrm{~nm} f=0.0237<S^{* *} 2>=0.804$ 

145B $->151 B \quad 0.28323$
146B $->151 B \quad-0.14509$
Excited State 25: 2.175-A
146A $>152 A \quad 0.10673$
$150 \mathrm{~A} \rightarrow 152 \mathrm{~A} \quad 0.74225$
$150 \mathrm{~A} \rightarrow 153 \mathrm{~A} \quad-0.26493$
$151 \mathrm{~A} \rightarrow 155 \mathrm{~A} \quad 0.12171$
$143 B->151 B \quad-0.36310$
150B $->153 B \quad 0.11618$
150B $->154 B \quad-0.17295$
150B $->155 B \quad 0.10439$

Excited State 26: 2.017-A 150A $\rightarrow 152 A \quad-0.17665$

$151 \mathrm{~A}->155 \mathrm{~A} \quad 0.66146$

150B $->155 B \quad 0.66635$

Excited State 27: 2.134-A 141A $>154 \mathrm{~A} \quad-0.10341$

$145 \mathrm{~A} \rightarrow 153 \mathrm{~A} \quad 0.15810$

$145 \mathrm{~A} \rightarrow 154 \mathrm{~A} \quad 0.14839$

$146 \mathrm{~A} \rightarrow 152 \mathrm{~A} \quad-0.11628$

$148 \mathrm{~A} \rightarrow 154 \mathrm{~A} \quad-0.10966$

$149 A->153 A \quad 0.11936$

149A $>154 A \quad 0.12784$

$150 \mathrm{~A} \rightarrow 153 \mathrm{~A} \quad 0.32995$

$150 \mathrm{~A} \rightarrow 154 \mathrm{~A} \quad-0.15083$

$139 B->151 B \quad-0.20392$

$142 B->151 B \quad 0.58148$

$142 B->154 B \quad 0.10000$

144B $->151 B \quad-0.17893$

$144 \mathrm{~B}->152 B \quad 0.12658$

$145 B->152 B \quad-0.10600$

145B $->153 B \quad-0.11191$

$145 B->154 B \quad-0.13431$

$148 \mathrm{~B}->152 \mathrm{~B} \quad 0.18316$

Excited State 28: 2.175-A
144A $\rightarrow 154 A$
0.11682
$147 A->153 A$
$-0.10342$
$148 \mathrm{~A} \rightarrow 152 \mathrm{~A}$
$-0.14364$
$148 \mathrm{~A} \rightarrow 153 \mathrm{~A}$
$-0.11021$
$148 \mathrm{~A} \rightarrow 154 \mathrm{~A}$
0.14137
$149 \mathrm{~A} \rightarrow 153 \mathrm{~A}$
0.10169
$149 \mathrm{~A} \rightarrow 155 \mathrm{~A}$
0.14363
$150 A->153 A$
$-0.27179$
$150 \mathrm{~A} \rightarrow 154 \mathrm{~A}$
0.30527
$140 \mathrm{~B}->152 \mathrm{~B}$
$-0.12574$
$142 B->151 B$
0.50904
$143 B->152 B$
0.10164
144B ->151B
$-0.14489$

2.9644 eV $418.25 \mathrm{~nm} f=0.0399\left\langle S^{* *} 2>=0.932\right.$

3.0110 eV $411.77 \mathrm{~nm} f=0.0877\left\langle S^{* *} 2>=0.768\right.$

3.1127 eV $398.31 \mathrm{~nm} f=0.0103\left\langle S^{* *} 2>=0.888\right.$

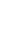

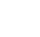




$$
\begin{array}{lr}
144 \mathrm{~B}->152 \mathrm{~B} & -0.13841 \\
144 \mathrm{~B}->154 \mathrm{~B} & 0.12544 \\
147 \mathrm{~B}->152 \mathrm{~B} & -0.20285 \\
148 \mathrm{~B}->152 \mathrm{~B} & -0.14744 \\
148 \mathrm{~B}->153 \mathrm{~B} & 0.10764 \\
148 \mathrm{~B}->154 \mathrm{~B} & 0.16352 \\
149 \mathrm{~B}->155 \mathrm{~B} & -0.14703 \\
150 \mathrm{~B}->152 \mathrm{~B} & -0.10872
\end{array}
$$

Excited State 29: 3.356-A
149A $>153 \mathrm{~A}$
0.12583
$149 \mathrm{~A}->155 \mathrm{~A}$
0.55373
149A $\rightarrow 159 A$
0.12248
150A $>>154 A$
$-0.11436$
$151 \mathrm{~A} \rightarrow>159 \mathrm{~A}$
$-0.24754$
$142 B->151 B$
$-0.13118$
$149 B->152 B$
$-0.17027$
$149 B->153 B \quad-0.20207$
149B $->155 B \quad-0.52878$
$149 \mathrm{~B}->158 \mathrm{~B} \quad-0.13128$
150B $->158 B$
0.24801

Excited State 30: 2.077-A $141 \mathrm{~A}->153 \mathrm{~A}$

0.15227

$145 \mathrm{~A} \rightarrow>153 \mathrm{~A}$

$-0.20742$

$145 \mathrm{~A} \rightarrow 154 \mathrm{~A}$

$-0.12027$

$148 \mathrm{~A} \rightarrow 152 \mathrm{~A}$

0.10464

$148 \mathrm{~A} \rightarrow 153 \mathrm{~A}$

0.10293

149A $>152 A$

$-0.16532$

149A $>>153 A$

$-0.28073$

149A $>154 A$

$-0.10426$

$150 \mathrm{~A} \rightarrow>152 \mathrm{~A}$

$-0.11075$

$150 \mathrm{~A}->153 \mathrm{~A}$

$-0.19944$

150A $>154 A$

$-0.24592$

$140 \mathrm{~B}->151 \mathrm{~B}$

0.10343

140B $->152 B$

0.14165

$141 B->151 B$

0.11198

$142 B->151 B$

0.38021

$142 B->152 B$

$-0.11109$

$144 \mathrm{~B}->151 \mathrm{~B}$

$-0.10851$

$145 B$ - $>152 B$

0.24362

$145 B->153 B$

0.18856

$147 \mathrm{~B}->152 \mathrm{~B}$

0.11888

149B $->152 B$

$-0.25145$

$149 B->153 B$

$-0.12920$

149B ->155B

0.13186

3.1787 eV $390.05 \mathrm{~nm} f=0.0003\left\langle S^{* *} 2>=2.566\right.$

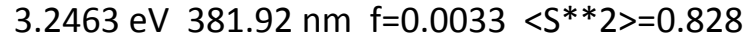

G) TD-DFT excitation energies and oscillator strengths for $)$ for high-spin $\left(5^{+}\right)_{2}(\mu-O A C)(S=2)$

Excitation energies and oscillator strengths:

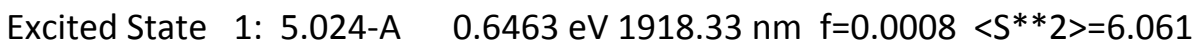




$\begin{array}{lr}252 B->278 B & -0.26022 \\ 253 B->278 B & -0.14028 \\ 260 B->278 B & -0.14555 \\ 264 B->278 B & 0.17459 \\ 265 B->278 B & 0.10931 \\ 267 B->278 B & 0.41784 \\ 271 B->277 B & -0.10445 \\ 271 B->278 B & 0.57472 \\ 272 B->278 B & -0.16586 \\ 273 B->278 B & 0.10999 \\ 274 B->278 B & -0.27674 \\ 275 B->278 B & -0.26230\end{array}$

This state for optimization and/or second-order correction.

Total Energy, E(TD-HF/TD-DFT) $=-5838.44135652$

Copying the excited state density for this state as the 1-particle RhoCl density.

\begin{tabular}{|c|c|}
\hline \multicolumn{2}{|c|}{ Excited State $2: 5.023-\mathrm{A}$} \\
\hline $252 B->277 B$ & 0.14341 \\
\hline $54 B->277 B$ & 0.17816 \\
\hline $255 B->277 B$ & 0.15558 \\
\hline $258 B->277 B$ & -0.17325 \\
\hline $260 B->277 B$ & -0.19398 \\
\hline $264 B->277 B$ & -0.11698 \\
\hline $265 B->277 B$ & -0.11083 \\
\hline $266 B->277 B$ & -0.13323 \\
\hline $268 B->277 B$ & 0.38775 \\
\hline $271 B->277 B$ & 0.17260 \\
\hline $272 B->277 B$ & 0.68950 \\
\hline $272 B->278 B$ & 0.10627 \\
\hline $276 B->277 B$ & 0.2000 \\
\hline
\end{tabular}

Excited State $3: 5.027-\mathrm{A} \quad 0.9378 \mathrm{eV} 1322.09 \mathrm{~nm} \quad \mathrm{f}=0.0011 \quad \mathrm{~S}^{* *} 2>=6.067$ $252 B->277 B \quad 0.12727$

$254 \mathrm{~B} \rightarrow>277 \mathrm{~B} \quad 0.20809$

$255 \mathrm{~B} \rightarrow>277 \mathrm{~B} \quad 0.14747$

$258 \mathrm{~B} \rightarrow>277 \mathrm{~B} \quad 0.17125$

$260 \mathrm{~B} \rightarrow>277 \mathrm{~B} \quad 0.13841$

$261 \mathrm{~B} \rightarrow>277 \mathrm{~B} \quad 0.17129$

$264 B->277 B \quad 0.20646$

$265 B \rightarrow 277 B \quad 0.23674$

$266 \mathrm{~B} \rightarrow>277 \mathrm{~B} \quad 0.40039$

$268 \mathrm{~B}->277 \mathrm{~B} \quad 0.49393$

$272 B->279 B \quad-0.15088$

$272 B->280 B \quad-0.20980$

$273 B->277 B \quad-0.21105$

$276 \mathrm{~B}->277 \mathrm{~B} \quad-0.18534$

Excited State $4: 5.027-\mathrm{A} \quad 0.9796 \mathrm{eV} 1265.70 \mathrm{~nm} \quad \mathrm{f}=0.0009<\mathrm{S}^{* *} 2>=6.067$ $252 \mathrm{~B}->278 \mathrm{~B} \quad-0.16443$

$253 \mathrm{~B}->278 \mathrm{~B} \quad-0.11823$

$254 \mathrm{~B}->278 \mathrm{~B} \quad 0.13236$

$255 \mathrm{~B}->278 \mathrm{~B} \quad 0.10638$

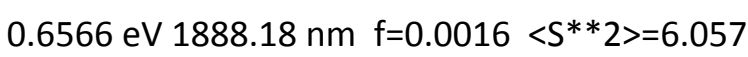

0.9378 eV $1322.09 \mathrm{~nm}+0.0011<\mathrm{S} * 2>6.067$

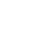




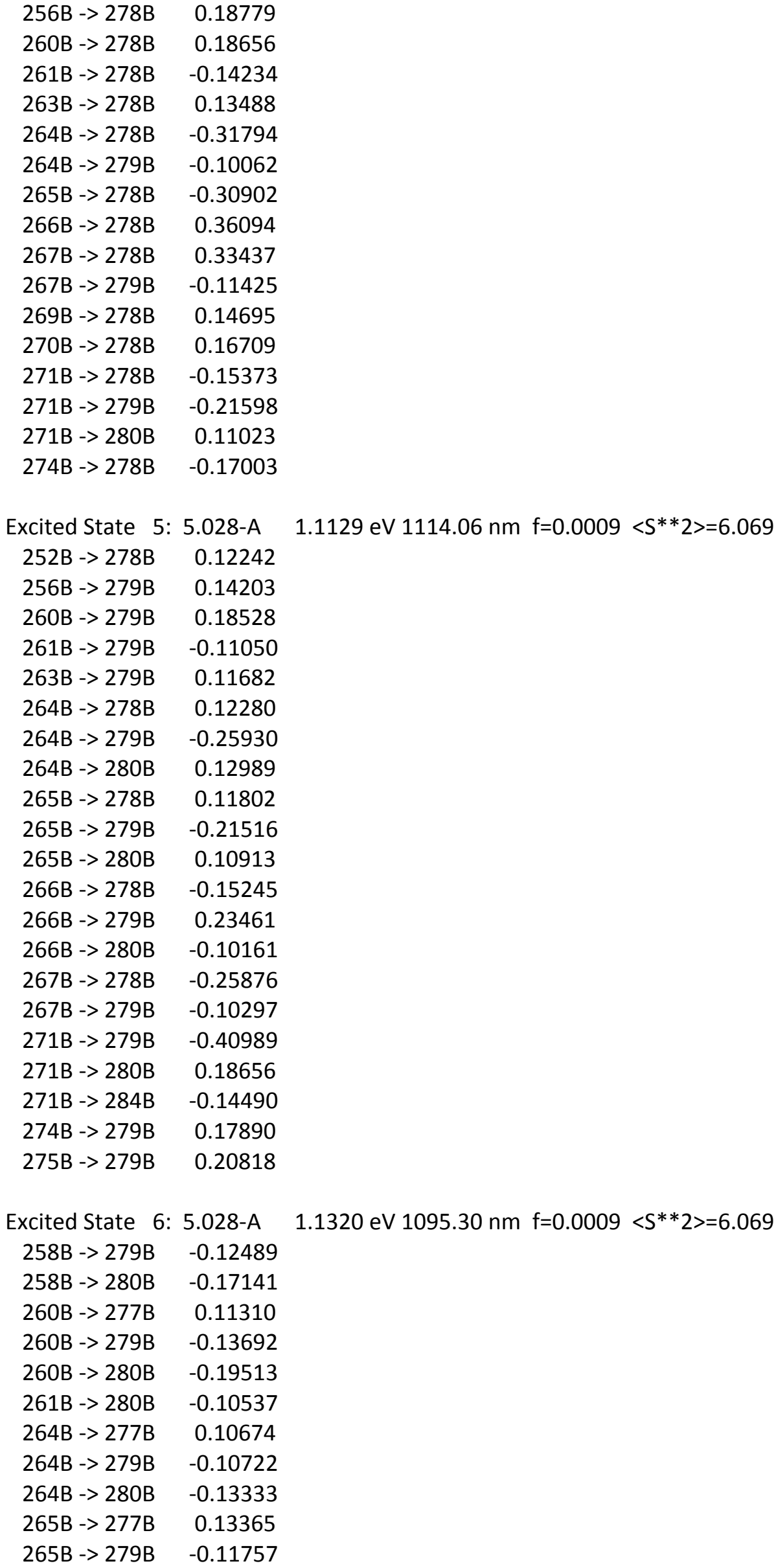




$$
\begin{array}{lr}
265 B->280 B & -0.14463 \\
266 B->277 B & 0.21343 \\
266 B->279 B & -0.15038 \\
266 B->280 B & -0.20821 \\
268 B->277 B & 0.24811 \\
271 B->280 B & 0.12281 \\
272 B->279 B & 0.30921 \\
272 B->280 B & 0.40437 \\
273 B->277 B & 0.34226 \\
276 B->280 B & 0.11522
\end{array}
$$

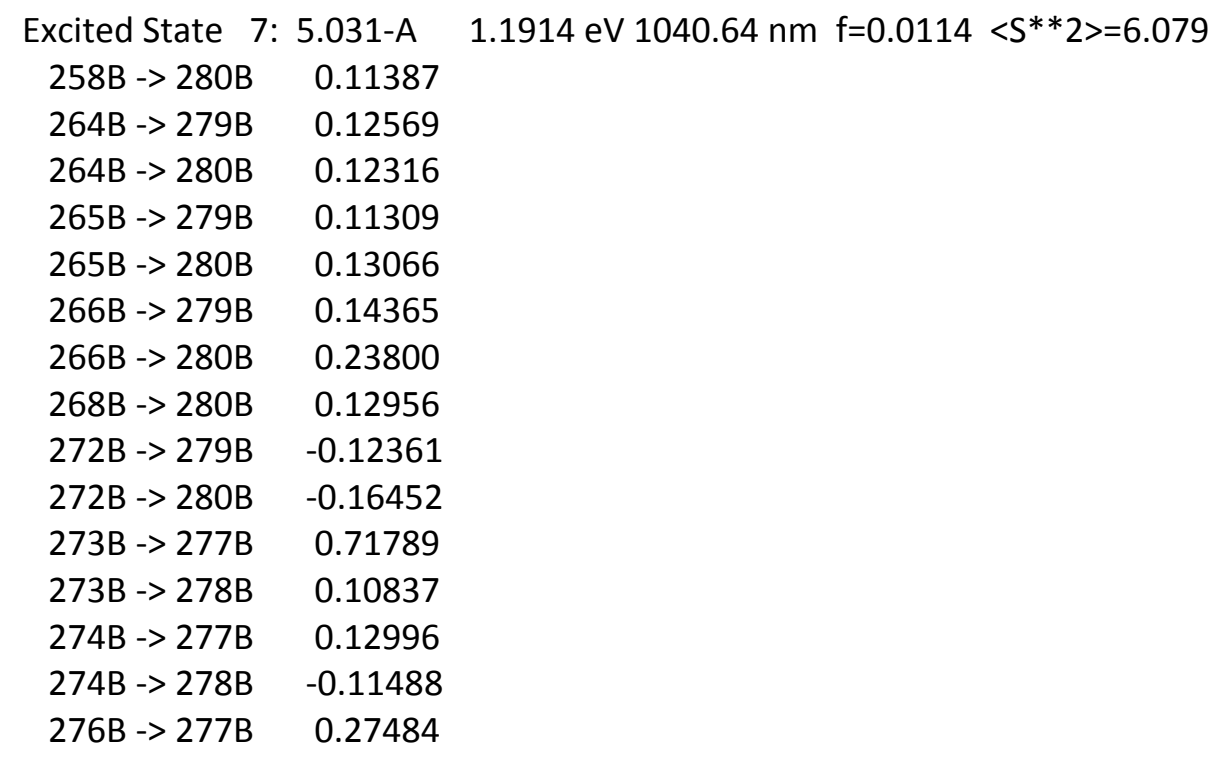

Excited State 8: 5.037-A $\quad 1.2951 \mathrm{eV} 957.34 \mathrm{~nm} \mathrm{f}=0.0094\left\langle\mathrm{~S}^{* *} 2\right\rangle=6.094$ 264B $>$ 279B $\quad-0.12086$

$266 \mathrm{~B} \rightarrow>279 \mathrm{~B} \quad 0.15830$

$267 \mathrm{~B}->278 \mathrm{~B} \quad 0.19699$

$267 \mathrm{~B}->279 \mathrm{~B} \quad 0.16138$

$271 B->278 B \quad 0.20697$

$273 B->277 B \quad 0.15535$

$274 \mathrm{~B} \rightarrow>277 \mathrm{~B} \quad-0.10640$

$274 B \rightarrow 278 B \quad 0.65039$

$275 B->278 B \quad 0.43779$

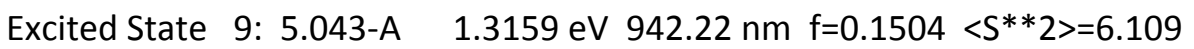

$266 \mathrm{~B}->277 \mathrm{~B} \quad 0.11584$

$272 B->277 B \quad-0.18461$

$273 B->277 B \quad-0.26722$

$276 \mathrm{~B}->277 \mathrm{~B} \quad 0.88044$

$276 \mathrm{~B}->278 \mathrm{~B} \quad 0.10499$

Excited State 10: $5.047-\mathrm{A} \quad 1.4117 \mathrm{eV} 878.26 \mathrm{~nm} \quad \mathrm{f}=0.1420<\mathrm{S}^{* *} 2>=6.118$

$274 \mathrm{~B} \rightarrow>277 \mathrm{~B}$

$274 \mathrm{~B}->278 \mathrm{~B} \quad-0.52359$

$275 B->277 B \quad-0.17828$

$275 B$ B $>278 B \quad 0.76538$ 
H) TD-DFT excitation energies and oscillator strengths for CoSal(OAC) adduct $(S=1)$

Excitation energies and oscillator strengths:

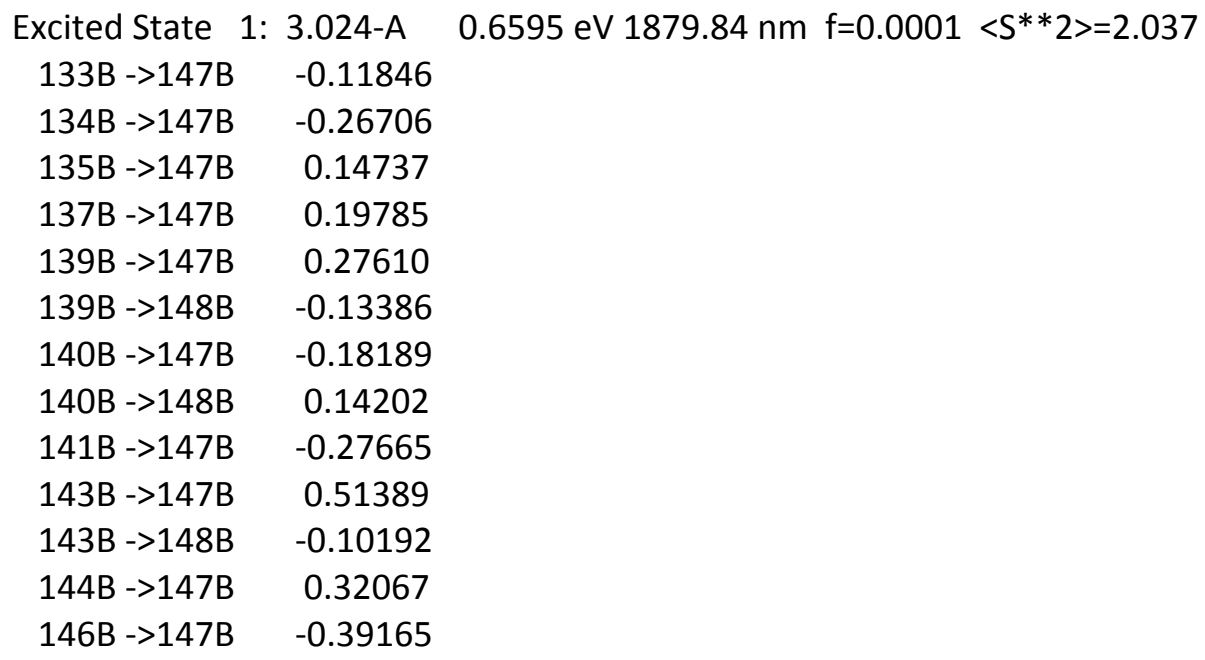

This state for optimization and/or second-order correction.

Total Energy, E(TD-HF/TD-DFT) = -3033.57354334

Copying the excited state density for this state as the 1-particle RhoCl density.

\begin{tabular}{|c|c|}
\hline \multicolumn{2}{|c|}{ Excited State 2: 3.034-A } \\
\hline $111 \mathrm{~B}->147 \mathrm{~B}$ & 0.10100 \\
\hline 127B ->147B & 0.10047 \\
\hline 133B ->147B & -0.12947 \\
\hline 134B ->147B & -0.31458 \\
\hline $134 \mathrm{~B}->148 \mathrm{~B}$ & 0.10256 \\
\hline $135 B->147 B$ & -0.29334 \\
\hline 137B ->147B & -0.13387 \\
\hline $137 \mathrm{~B}->148 \mathrm{~B}$ & -0.10024 \\
\hline 139B ->147B & -0.30984 \\
\hline $140 B->$ & 0.46971 \\
\hline 141B ->147B & -0.40245 \\
\hline $141 B->148 B$ & 0.10426 \\
\hline $142 B->147 B$ & -0.20176 \\
\hline $143 B->148 B$ & -0.19749 \\
\hline $144 B->148 B$ & -0.12481 \\
\hline $146 \mathrm{~B}->148$ & 0.145 \\
\hline
\end{tabular}

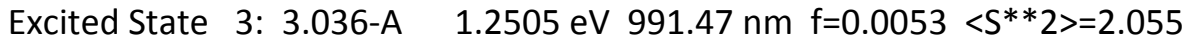

$\begin{array}{lr}134 \mathrm{~B}->147 \mathrm{~B} & 0.17069 \\ 134 \mathrm{~B}->148 \mathrm{~B} & 0.10302 \\ 135 \mathrm{~B}->148 \mathrm{~B} & -0.18018 \\ 137 \mathrm{~B}->148 \mathrm{~B} & -0.19863 \\ 139 \mathrm{~B}->148 \mathrm{~B} & -0.29875 \\ 139 \mathrm{~B}->150 \mathrm{~B} & 0.13936 \\ 140 \mathrm{~B}->147 \mathrm{~B} & -0.14946\end{array}$

0.9994 eV $1240.63 \mathrm{~nm} f=0.0002\left\langle S^{* *} 2>=2.051\right.$ 


$$
\begin{array}{lr}
140 B->148 B & 0.29126 \\
140 B->150 B & -0.12280 \\
141 B->147 B & 0.21957 \\
142 B->148 B & -0.10385 \\
143 B->147 B & -0.12605 \\
143 B->148 B & -0.37411 \\
143 B->150 B & 0.18784 \\
144 B->148 B & -0.25151 \\
144 B->150 B & 0.12464 \\
146 B->148 B & 0.39435 \\
146 B->150 B & -0.12766
\end{array}
$$

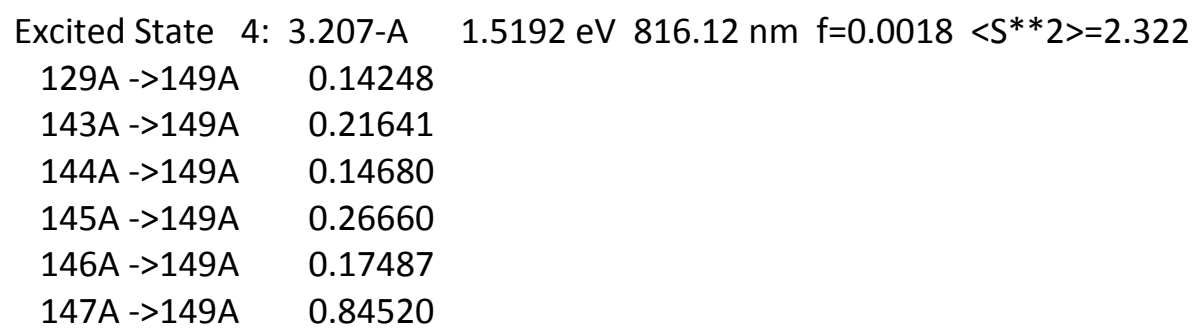

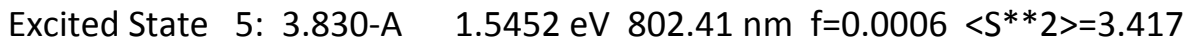
$131 \mathrm{~A}->149 \mathrm{~A}$
0.10872
$137 A->149 A$
$-0.23068$
$139 A->149 A$
$-0.11250$
$140 \mathrm{~A} \rightarrow 149 \mathrm{~A}$
0.15167
$141 \mathrm{~A} \rightarrow>149 \mathrm{~A}$
$-0.15105$
$142 \mathrm{~A} \rightarrow 149 \mathrm{~A}$
0.15767
$143 A->149 A$
0.12687
$144 A->149 A$
0.16242
$145 \mathrm{~A} \rightarrow 149 \mathrm{~A}$
$-0.12965$
$147 \mathrm{~A} \rightarrow 149 \mathrm{~A}$
0.11369
$148 \mathrm{~A} \rightarrow 149 \mathrm{~A}$
0.71817
$134 \mathrm{~B}->151 B$
$-0.11665$
139B $->151 B$
0.11127
$141 B->151 B$
$-0.11483$
143B $->150 B$
$-0.10106$
$143 B->151 B$
0.20207
$144 B->151 B$
0.12596
146B ->151B
$-0.11015$

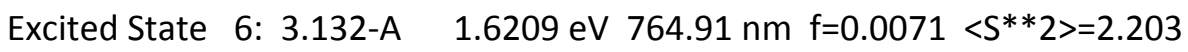
$134 \mathrm{~B}->148 \mathrm{~B}$
$-0.17212$
$135 \mathrm{~B}->147 \mathrm{~B}$
0.12718
$135 \mathrm{~B}->148 \mathrm{~B}$
$-0.10356$
$140 \mathrm{~B}->148 \mathrm{~B}$
0.16498
$141 \mathrm{~B}->148 \mathrm{~B}$
$-0.20992$
$143 B->147 B$
0.17369
$144 B->147 B$
0.16015
$145 B->147 B$
$-0.16980$
146B ->147B
0.79412

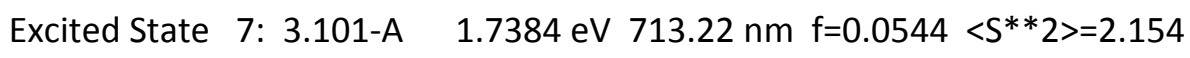




$$
\begin{array}{lr}
148 \mathrm{~A}->149 \mathrm{~A} & 0.19164 \\
136 \mathrm{~B}->147 \mathrm{~B} & -0.10766 \\
145 \mathrm{~B}->147 \mathrm{~B} & 0.90347 \\
146 \mathrm{~B}->147 \mathrm{~B} & 0.18507 \\
146 \mathrm{~B}->148 \mathrm{~B} & -0.12642
\end{array}
$$

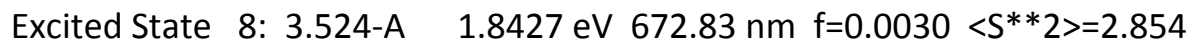

$$
\begin{array}{ll}
132 A->149 A & -0.13472 \\
142 A->149 A & -0.25438 \\
143 A->149 A & -0.16759 \\
146 A->149 A & 0.10892 \\
148 A->149 A & 0.49601 \\
134 B->148 B & -0.18058 \\
134 B->151 B & 0.22119 \\
140 B->148 B & 0.16979 \\
141 B->148 B & -0.24564 \\
141 B->151 B & 0.24744 \\
143 B->148 B & 0.14647 \\
143 B->151 B & -0.23238 \\
144 B->151 B & -0.11469 \\
145 B->147 B & -0.20951 \\
146 B->147 B & -0.15341 \\
146 B->148 B & -0.18783 \\
146 B->151 B & 0.15086
\end{array}
$$

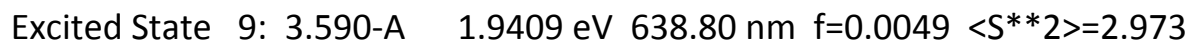

$$
\begin{array}{lc}
137 \mathrm{~A}->149 \mathrm{~A} & 0.31445 \\
138 \mathrm{~A}->149 \mathrm{~A} & 0.12322 \\
143 \mathrm{~A}->149 \mathrm{~A} & -0.13914 \\
146 \mathrm{~A}->149 \mathrm{~A} & 0.10776 \\
148 \mathrm{~A}->149 \mathrm{~A} & 0.31086 \\
134 \mathrm{~B}->148 \mathrm{~B} & 0.20052 \\
134 \mathrm{~B}->151 \mathrm{~B} & -0.11374 \\
135 \mathrm{~B}->148 \mathrm{~B} & 0.10906 \\
135 \mathrm{~B}->151 \mathrm{~B} & -0.17970 \\
137 \mathrm{~B}->151 \mathrm{~B} & -0.11143 \\
139 \mathrm{~B}->148 \mathrm{~B} & 0.15735 \\
139 \mathrm{~B}->151 \mathrm{~B} & -0.22107 \\
140 \mathrm{~B}->148 \mathrm{~B} & -0.25420 \\
140 \mathrm{~B}->151 \mathrm{~B} & 0.28382 \\
141 \mathrm{~B}->148 \mathrm{~B} & 0.28753 \\
141 \mathrm{~B}->151 \mathrm{~B} & -0.13500 \\
142 \mathrm{~B}->151 \mathrm{~B} & -0.10554 \\
143 \mathrm{~B}->147 \mathrm{~B} & 0.12681 \\
143 \mathrm{~B}->148 \mathrm{~B} & -0.12144 \\
145 \mathrm{~B}->147 \mathrm{~B} & -0.17316 \\
146 \mathrm{~B}->147 \mathrm{~B} & 0.20385 \\
146 \mathrm{~B}->148 \mathrm{~B} & -0.10943 \\
146 \mathrm{~B}->151 \mathrm{~B} & 0.12940
\end{array}
$$

Excited State 10: $3.658-\mathrm{A} \quad 2.1042 \mathrm{eV} 589.21 \mathrm{~nm} \mathrm{f}=0.0133<\mathrm{S}^{* *} 2>=3.096$ $126 \mathrm{~A} \rightarrow 149 \mathrm{~A} \quad-0.20619$ 


\begin{tabular}{|c|c|}
\hline $132 A->149 A$ & -0.24573 \\
\hline $137 A->149 A$ & -0.15693 \\
\hline $138 \mathrm{~A}->149 A$ & -0.10073 \\
\hline $142 A->149 A$ & -0.29791 \\
\hline $147 A->149 A$ & -0.10074 \\
\hline $147 A->150 A$ & -0.17829 \\
\hline $148 \mathrm{~A} \rightarrow 150 \mathrm{~A}$ & -0.12528 \\
\hline $148 \mathrm{~A}->151 \mathrm{~A}$ & -0.13661 \\
\hline $134 B->148 B$ & 0.14090 \\
\hline $134 B->150 B$ & -0.11639 \\
\hline $134 B->151 B$ & 0.11922 \\
\hline $135 B->148 B$ & 0.14869 \\
\hline 135B $->150 B$ & -0.10047 \\
\hline $139 B->148 B$ & 0.11705 \\
\hline $139 B->150 B$ & -0.11412 \\
\hline $139 B->151 B$ & 0.10716 \\
\hline $140 B->148 B$ & -0.20723 \\
\hline $140 B->150 B$ & 0.17949 \\
\hline $140 B->151 B$ & -0.16451 \\
\hline $141 B->148 B$ & 0.20509 \\
\hline $141 B->150 B$ & -0.15478 \\
\hline $141 B->151 B$ & 0.15798 \\
\hline $142 B->148 B$ & 0.10583 \\
\hline $145 B->149 B$ & 0.11083 \\
\hline $146 B$->147B & 0.13386 \\
\hline $146 B->148 B$ & 0.4063 \\
\hline
\end{tabular}

